\title{
Electrochemical Testing Of Ni-Cr-Mo-Gd Alloys
}

\author{
T. E. Lister \\ R. E. Mizia \\ S. M. Birk
}

October 2005

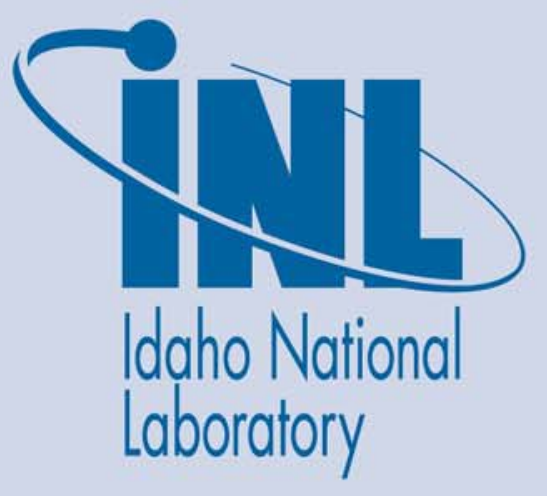

The INL is a U.S. Department of Energy National Laboratory operated by Battelle Energy Alliance 



\section{Electrochemical Testing of Ni-Cr-Mo-Gd Alloys}

T. E. Lister

R. E. Mizia

S. M. Birk

October 2005

Idaho National Laboratory

Nuclear Materials Disposition and Engineering

Idaho Falls, Idaho 83415

Prepared for the

U.S. Department of Energy

Office of Civilian Radioactive Waste Management

Under DOE Idaho Operations Office

Contract DE-AC07-05ID14517 



\title{
Electrochemical Testing of Ni-Cr-Mo-Gd Alloys
}

\author{
INL/EXT-05-00713
}

\section{October 2005}

\section{Approved by}

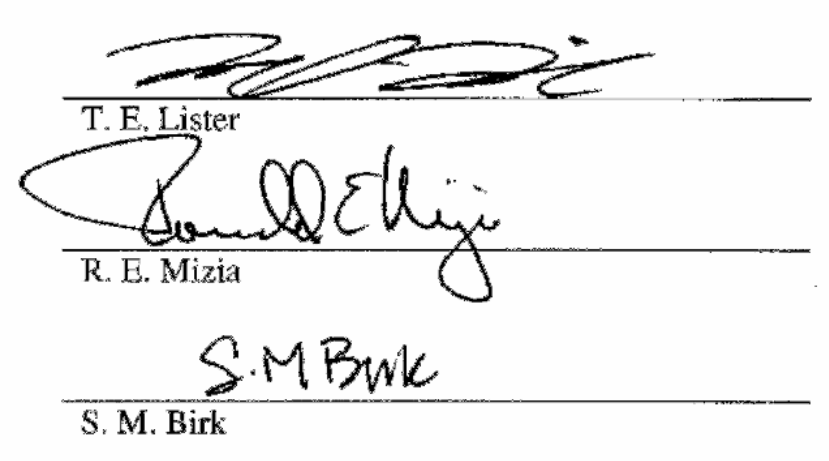

$\frac{10 / 25 / 05}{\text { Date }}$
$\frac{10 / 25 / 05}{\text { Date }}$
$\frac{10 / 25 / 05}{\text { Date }}$





\begin{abstract}
The waste package site recommendation design specified a boron-containing stainless steel, Neutronit 976/978, for fabrication of the internal baskets that will be used as a corrosion-resistant neutron-absorbing material. Recent corrosion test results gave higher-than-expected corrosion rates for this material. The material callout for these components has been changed to a Ni-Cr-Mo-Gd alloy (ASTM-B 932-04, UNS N06464) that is being developed at the Idaho National Laboratory.

This report discusses the results of initial corrosion testing of this material in simulated in-package environments that could contact the fuel baskets after breach of the waste package outer barrier. The corrosion test matrix was executed using the potentiodynamic and potentiostatic electrochemical test techniques.

The alloy performance shows low rates of general corrosion after initial removal of a gadolinium-rich second phase that intersects the surface. The high halide-containing test solutions exhibited greater tendencies toward initiation of crevice corrosion.
\end{abstract}




\section{CONTENTS}

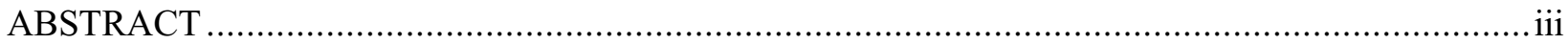

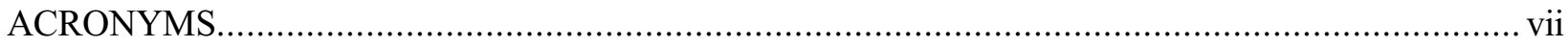

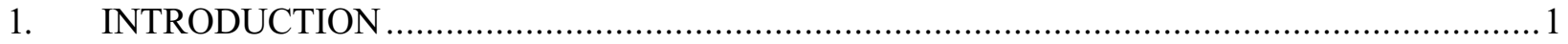

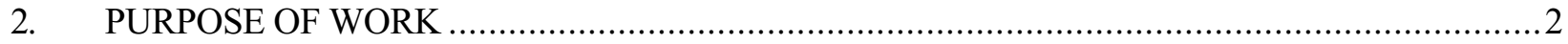

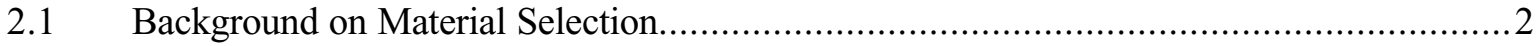

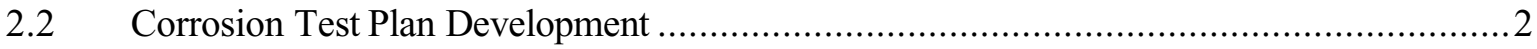

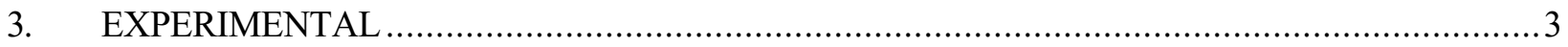

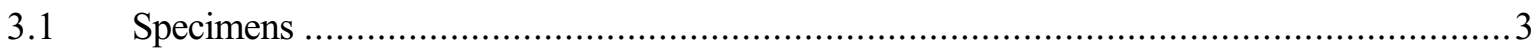

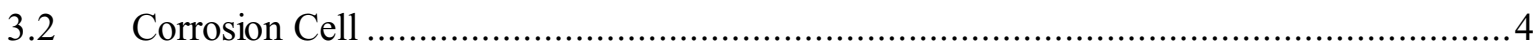

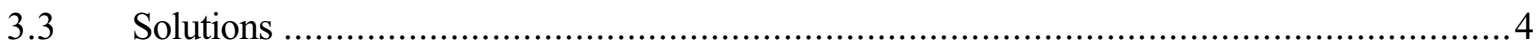

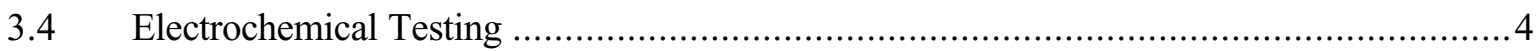

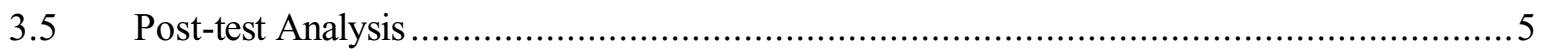

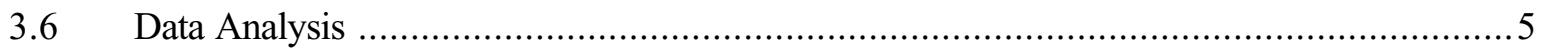

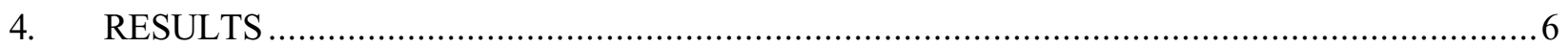

4.1 Corrosion Potential Measurements ..................................................................... 6

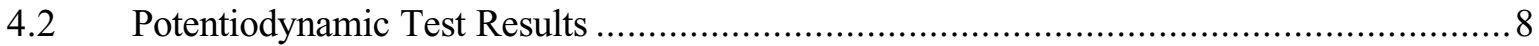

4.2.1 Explanation of Potentiodynamic Data.............................................. 8

4.2.2 Potentiodynamic Tests Results........................................................... 9

4.3 Linear Polarization Resistance Measurements....................................................... 15

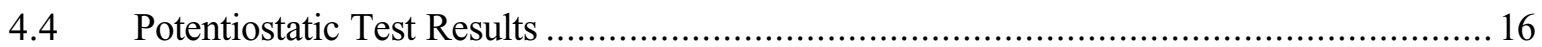

4.4.1 Explanation of Potentiostatic Tests..................................................... 16

4.4.2 Potentiostatic Test Results .............................................................. 17

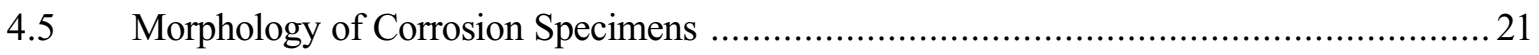

4.6 Analysis of Corrosion Test Solutions ....................................................................... 23

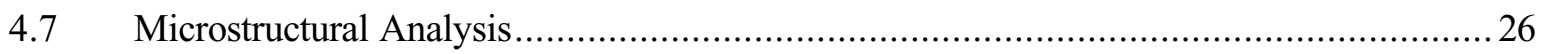

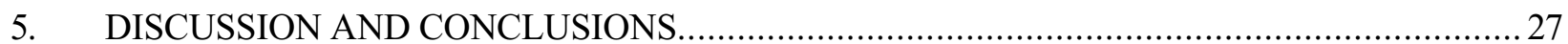


$5.1 \quad$ Discussion of Results

5.2 Future Work

6. REFERENCES.

\section{FIGURES}

1. Diagram of a hypothetical potentiodynamic curve for Ni-Cr-Mo-Gd alloy demonstrating the various parameters collected from the curves

2. Potentiodynamic curves for Tests 042605 and 072605 . See Tables 4 and 5 for conditions

3. Potentiostatic curves for Tests 063005 and 072605 . See Tables 7 and 8 for conditions

4. Diagram of a hypothetical potentiostatic curve for Ni-Cr-Mo-Gd alloy demonstrating two regions of behavior

5. Photograph of the crevice specimen from Test 063005 showing extensive crevice attack.

6. SEM micrographs of test specimens: (a) Test 063005 specimen from boldly exposed region,

(b) Test 072505 specimen, and (c) high magnification of pot from Test 042605 specimen.

7. SEM micrographs of crevice damage on Test 063005 specimen at various magnifications.

8. Light optical micrograph of cross-sectioned specimen at 500X magnification .........................26

9. Diagram of Ni-Cr-Mo-Gd surface evolution during potentiostatic tests.

\section{TABLES}

1. Composition of Ni-Cr-Mo-Gd alloy M340 3

2. Composition of solutions used in corrosion testing 4

3. Corrosion potential data (vs. SCE) collected prior to each electrochemical test........................6

4. Data obtained from potentiodynamic testing of cylindrical specimens ................................... 13

5. Data obtained from potentiodynamic testing of crevice specimens...................................... 14

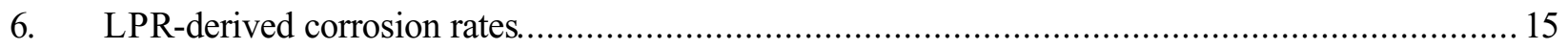

7. Data obtained from potentiostatic testing of cylindrical specimens .................................. 18

8. Data obtained from potentiostatic testing of crevice specimens ....................................... 19

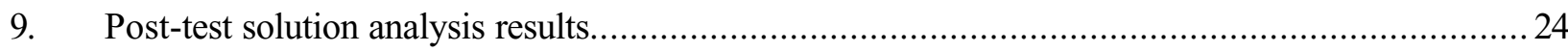




\section{ACRONYMS}

\begin{tabular}{|c|c|}
\hline ASME & American Society of Mechanical Engineers \\
\hline ASTM & American Society of Testing and Materials \\
\hline $\mathrm{BSC}$ & Bechtel SAIC, LLC \\
\hline DOE & U.S. Department of Energy \\
\hline $\mathrm{E}_{\text {corr }}$ & corrosion potential \\
\hline $\mathrm{E}_{\text {crit }}$ & critical potential \\
\hline $\mathrm{E}_{\mathrm{rp}}$ & repassivation potential \\
\hline ICP-AES & inductively coupled plasma atomic emission spectroscopy \\
\hline INL & Idaho National Laboratory \\
\hline LOM & light optical microscopy \\
\hline LPR & linear polarization resistance \\
\hline MCA & multiple crevice assembly \\
\hline NSNFP & National Spent Nuclear Fuel Program \\
\hline PD & potentiodynamic \\
\hline PS & potentiostatic \\
\hline SCE & saturated calomel electrode \\
\hline SEM & scanning electron microscopy \\
\hline SNF & spent nuclear fuel \\
\hline $\mathrm{SSC}$ & saturated silver chloride \\
\hline YMP & Yucca Mountain Project \\
\hline
\end{tabular}




\section{Electrochemical Testing of Ni-Cr-Mo-Gd Alloys 1. INTRODUCTION}

A corrosion-resistant, neutron-absorbing material based on the Ni-Cr-Mo alloy system with a gadolinium addition is being developed by the Idaho National Laboratory (INL). This work is being managed by the National Spent Nuclear Fuel Program (NSNFP), which coordinates and integrates national efforts to manage and dispose of U.S. Department of Energy (DOE)-owned spent nuclear fuel (SNF) for DOE Office of Environmental Management. The material is described in an American Society for Testing and Materials (ASTM) standard B932-04. ${ }^{1}$ An American Society of Mechanical Engineers (ASME) Code Case N-728 for use of the material in Section III, Division 3 is in the final approval process. $^{2}$

The metallurgical development process and corrosion performance of this material has been reported previously. ${ }^{3-8}$ This material has an austenitic microstructure with a eutectic second phase of the form $\mathrm{Ni}_{5} \mathrm{Gd}$, which is called a gadolinide. The size, shape, and distribution of the gadolinide in the plate

product are controlled by alloy chemistry, primary and secondary melting/refining, and thermomechanical processing. This secondary phase was preferentially removed in electrochemical and long-term immersion corrosion tests leaving a passivated surface. The passivated surface then tends to result in corrosion performance similar to other Ni-Cr-Mo alloys in the test solutions.

Under Bechtel SAIC, LLC (BSC) technical direction, a corrosion testing program for this new material was initiated in FY 2005 by INL. BSC provided INL with the required test parameters such as temperature, environment, and specimen configuration. The INL-prepared test program was designed to provide confirming corrosion rate data over the range of relevant waste package, in-package environments, and temperatures potentially present once the waste package is breached. Another goal of the testing program was to demonstrate that the gadolinium-rich secondary phase (gadolinide) in this material will not be leached from the matrix through localized corrosion processes after the initial removal of the gadolinides that intersect the surface. 


\section{PURPOSE OF WORK}

\subsection{Background on Material Selection}

The BSC materials staff recommended boron-containing austenitic stainless steel, Neutronit 976/978, for the corrosion-resistant, neutron-absorbing plates used to fabricate the waste package fuel basket. This material can be characterized as a 316 stainless steel with a boron addition. Initial corrosion testing performed by Lawrence Livermore National Laboratory on a borated stainless steel gave favorable results. ${ }^{9}$ However, the corrosion rates measured on the Neutronit material at the Long-term Corrosion Test Facility located at Lawrence Livermore National Laboratory were higher than expected. ${ }^{10,11}$ The BSC materials staff then decided that the Ni-Cr-Mo-Gd alloy under development at INL was a promising replacement. An initial corrosion rate of $1 \mu \mathrm{m} /$ year was recommended for Yucca Mountain Project (YMP) modeling purposes based on analysis of published corrosion data on the Ni-Cr-Mo-Gd alloy and Alloy C-4.

\subsection{Corrosion Test Plan Development}

The BSC and INL staff discussed optimized corrosion test parameters and developing detailed descriptions of expected in-package environments that might contact the basket plates. The key statement in the resultant Statement of Work issued to the INL ${ }^{12}$ is "The proposed testing will provide confirming corrosion rate data over the range of relevant in-package environments and temperatures potentially present once the waste package is breached. The testing will also assist in demonstrating that a noninterconnected gadolinide particle is achie vable."

To execute the corrosion testing program and provide confirmatory corrosion rate data as described in the YMP Statement of Work, INL developed a Quality Plan, ${ }^{13}$ Experimental Plan, ${ }^{14}$ and an Experimental Procedures Plan. ${ }^{15}$ 


\section{EXPERIMENTAL}

The investigations were performed using procedures written specifically for the testing. These procedures are grouped together in a controlled document PLN-1885, "Experimental Procedures for Electrochemical Testing of Ni-Cr-Mo-Gd Alloys," (see Reference 15). These procedures were written to perform the work described in PLN-1880, "Experimental Plan for Electrochemical Testing of Ni-Cr-Mo-Gd Alloys," (see Reference 14).

\subsection{Specimens}

The specimens used for electrochemical testing were prepared from heat M340 (Table 1), which was also used for ASME Code qualification. The material ingots were prepared by Vacuum Induction Melting with a secondary refinement treatment using the Vacuum Arc Remelt process. The material was hot rolled to size, annealed at $1,204^{\circ} \mathrm{C}\left(2,200^{\circ} \mathrm{F}\right)$, and water quenched. Two specimen configurations were machined: crevice specimens $\left(\sim 15.8 \mathrm{~cm}^{2}\right.$ area $)$ and cylindrical specimens $\left(\sim 8.9 \mathrm{~cm}^{2}\right)$. The specimens were carefully controlled to avoid loss of traceability and were stored individually in a controlled cabinet (PLN-1885-Procedure 1100, Reference 15). The specimens were removed as needed for testing. Using ASTM G5 practice, ${ }^{16}$ the outer surfaces of the specimens were sequentially ground with 240 and 600 grit $\mathrm{SiC}$ paper (PLN-1885 Procedure 1500, Reference 15). The surface area was calculated from measurements of the specimen dimensions using calibrated calipers. Then the specimens were degreased (acetone and ethanol) and weighed prior to testing. The procedure called for careful handling practices and documentation with photographs to avoid mixing the specimens. Crevice tests used multiple crevice assembly (MCA) ceramic washers $(12$ tooth, OD $1.6 \mathrm{~cm}$, ID $0.65 \mathrm{~cm})$ with Teflon tape $(1-1 / 2-$ in.-wide military grade) placed between the specimen surface and the crevice washer. The crevice bolt was C-276 and tightened to 70 in.- $\mathrm{lb}$ (PLN-1885 Procedure 1600, Reference 15). Teflon compression washers were used to connect the assembly to the specimen holder. Cylindrical specimens used Viton rubber gaskets to seal the attachment of the specimen.

Table 1. Composition of Ni-Cr-Mo-Gd alloy M340.

\begin{tabular}{|c|c|}
\hline Element & $\begin{array}{c}\text { Weight } \\
(\%)\end{array}$ \\
\hline $\mathrm{Mo}$ & 14.07 \\
\hline $\mathrm{Cr}$ & 15.25 \\
\hline $\mathrm{Gd}$ & 1.99 \\
\hline $\mathrm{Co}$ & $<0.005$ \\
\hline $\mathrm{Mn}$ & $<0.005$ \\
\hline $\mathrm{Si}$ & 0.01 \\
\hline $\mathrm{Fe}$ & $<0.005$ \\
\hline $\mathrm{P}$ & $<0.005$ \\
\hline $\mathrm{O}$ & 0.0146 \\
\hline $\mathrm{N}$ & 0.0007 \\
\hline $\mathrm{C}$ & 0.0118 \\
\hline $\mathrm{S}$ & Not reported \\
\hline $\mathrm{Ni}$ & Balance \\
\hline
\end{tabular}




\subsection{Corrosion Cell}

The corrosion cell was a standard 1-L glass flask as described in ASTM G5 (Reference 16). PLN-1885 Attachments 14 and 15 show pictures of the complete cell setup. The testing stations have labels to avoid traceability issues. The cell and associated parts were carefully acid cleaned prior to testing to remove residual contaminates. The cell used water jacketed Luggin capillary compartments (with chilled water flow) to keep the reference electrode near room temperature during the test. A carbon rod was used as the counter electrode, and saturated calomel electrodes (SCEs) were used as reference electrodes. The reference electrodes were checked (PLN-1885 Procedure 1300, Reference 15) prior to each test. The solution temperature was controlled using a mantle heater setup with a thermocouple probe. The temperature of the solution and reference compartment was checked prior to initiating the test by a calibrated thermometer (PLN-1885 Procedure 1400, Reference 15). The cell also employed an Allin condenser with chilled water circulation to prevent solution loss. The cells were purged with nitrogen gas prior to and during the tests.

\subsection{Solutions}

The six solution compositions were defined in PLN-1880, Table 1 (see Reference 14). Only four of these solutions, Average low pH, High nitrate, High chloride, and High halide, were tested. These solutions were made in approximately 20 -L volumes. Immediately after being mixed, aliquots were taken in environmentally cleaned containers for later solution analysis. The anion targets were met using sodium salts. Nanopure deionized water was used in all solutions. The $\mathrm{pH}$ was achieved using additions of $\mathrm{HCl}$ and a standardized $\mathrm{pH}$ meter (PLN-1885 Procedures 1200 and 1700, Reference 15). Table 2 shows the ionic compositions obtained from analysis.

\subsection{Electrochemical Testing}

The setup and breakdown of the electrochemical tests were performed using detailed procedures (PLN-1885 Procedures 1500 and 2100, Reference 15). These setup and breakdown procedures had checklists (PLN-1885 Attachments 2-3, Reference 15) to ensure all steps were performed. The electrochemical tests were performed using calibrated potentiostats (PLN-1885 Procedure 4100, Reference 15) manufactured by Gamry Instruments. Two types of tests were performed: potentiostatic (PS) and potentiodynamic (PD). Each test type has a specific sequence of measurements that was maintained throughout all the tests. Both tests began with a 50-minute $\mathrm{E}_{\text {corr }}$ (open circuit potential) measurement. PS tests were performed for 7 days immediately following the $\mathrm{E}_{\text {corr }}$ measurement. For PD tests, a linear polarization (LPR) measurement was performed immediately following the $\mathrm{E}_{\text {corr }}$ measurement. This was performed at $600 \mathrm{mV} / \mathrm{hr}$ in the range of $\pm 30 \mathrm{mV}$ of the $\mathrm{E}_{\text {corr }}$ value. Then two PD scans (cyclic PD) were performed sequentially. The second PD test evaluates the performance after the expected dissolution of the secondary phase of the Ni-Cr-Mo-Gd alloy.

Table 2. Composition of solutions used in corrosion testing.

\begin{tabular}{|c|l|c|c|c|c|c|c|c|}
\hline Solution ID & Solution name & $\mathrm{pH}$ & $\begin{array}{c}{[\mathrm{Cl}]} \\
\mathrm{mg} / \mathrm{L}\end{array}$ & $\begin{array}{c}{\left[\mathrm{NO}_{3}^{-}\right]} \\
\mathrm{mg} / \mathrm{L}\end{array}$ & $\begin{array}{c}{\left[\mathrm{F}^{-}\right]} \\
\mathrm{mg} / \mathrm{L}\end{array}$ & $\begin{array}{c}{\left[\mathrm{Cl}^{-}\right]} \\
\mathrm{m}\end{array}$ & $\begin{array}{c}{\left[\mathrm{NO}_{3}^{-}\right]} \\
\mathrm{m}\end{array}$ & $\begin{array}{c}{\left[\mathrm{F}^{-}\right]} \\
\mathrm{m}\end{array}$ \\
\hline 100 & Average low $\mathrm{pH}$ & 5.71 & 134 & 27.5 & ND & $3.79 \mathrm{E}-03$ & $4.45 \mathrm{E}-04$ & ND \\
\hline 101 & High halide & 4.95 & 223 & 8.94 & 178 & $6.31 \mathrm{E}-03$ & $1.45 \mathrm{E}-04$ & $9.40 \mathrm{E}-03$ \\
\hline 102 & High nitrate & 5.07 & 71.8 & 47.2 & ND & $2.03 \mathrm{E}-03$ & $7.64 \mathrm{E}-04$ & ND \\
\hline 103 & High chloride & 5.36 & 218 & 9.06 & ND & $6.17 \mathrm{E}-03$ & $1.47 \mathrm{E}-04$ & ND \\
\hline
\end{tabular}




\subsection{Post-test Analysis}

The solution and specimen were examined using visual examination and photography. The specimen was descaled $(300-\mathrm{mL} \mathrm{HCl}$ in $1-\mathrm{L})$ to better elucidate damage and determine the weight loss due to the testing. An aliquot of solution was collected from the corrosion flask for later analysis of dissolved metal ion composition. The specimen was further examined by light optical microscopy (LOM) (PLN-1885 Procedure 3100, Reference 15) and scanning electron microscopy (SEM) (PLN-1885 Procedure 3200, Reference 15). Microstructural analysis was also performed for one specimen. The specimens were placed in archival storage for later retrieval if needed.

\subsection{Data Analysis}

Data were plotted using Igor Pro v. 4.01 and CorelDraw v. 11 (PLN-1885 Procedure 2700, Reference 15). These data plots are contained in the laboratory notebooks. The data were examined using validated software package Gamry Echem Analyst V1.31 supplied with the potentiostats. This software was used to integrate, find maximum and minimum values, and curve fit LPR data. Data workup sheets (PLN-1885 Attachments 4-5, Reference 15) were filled out for each test and pasted in the laboratory notebooks.

The current and charge data were scaled to the measured surface area. $\mathrm{E}_{\text {corr }}$ data were examined for initial, final, minimum, and maximum values (PLN-1885 Procedure 2500, Reference 15). LPR data were fitted to determine the polarization resistance from which the corrosion rate was calculated using ASTM G59 (PLN-1885 Procedure 1600, Reference 15). The generic Stern-Geary coefficient of $0.0261 \mathrm{~V}$ was used in this calculation (assume Tafel slopes of $0.12 \mathrm{~V} /$ decade). PD data were analyzed to determine a) the critical potential using crossover potential criteria, b) the charge in the passive and transpassive regions, and c) the maximum current in the passive and transpassive regions (PLN-1885 Procedure 2200, Reference 15). The division between the passive and transpassive regions was set at $0.6 \mathrm{~V}$ for the analysis of all tests. PS data were analyzed to determine: a) the total charge, b) the maximum current, and c) the current at the final data point defined as $\mathrm{I}_{\text {corr }}$ from which a corrosion rate is calculated (PLN-1885 Procedure 2300, Reference 15). The corrosion rate for PS tests was also determined using the weight loss due to the test. All potentials are reported versus the SCE. 


\section{RESULTS}

\subsection{Corrosion Potential Measurements}

The corrosion potential $\left(\mathrm{E}_{\mathrm{corr}}\right)$ was measured for 50 minutes before each electrochemical test. As the setup was identical for both types of tests (PD and PS), they can be compared as a whole. Four $\mathrm{E}_{\text {corr }}$ values were obtained from the $\mathrm{E}_{\text {corr }}$ data, initial, final, and minimum and maximum values. The value considered most relevant is the final value, as it represents the most equilibrated result. Although outside the scope of work, longer term $\mathrm{E}_{\text {corr }}$ measurements would be more ideal in understanding where the long-term $\mathrm{E}_{\text {corr }}$ value will equilibrate.

The values for $\mathrm{E}_{\text {corr }}$ obtained from the test data are grouped by the specimen type and environment, and are shown in Table 3. In general, $\mathrm{E}_{\text {corr }}$ values do not have a dependence on the specimen type or temperature. However, $\mathrm{E}_{\text {corr }}$ is strongly tied to the solution composition, with the solutions with higher halide concentrations having more negative corrosion potentials. The fluoride containing solution, High halide, has the lowest $\mathrm{E}_{\text {corr }}$ values. The Average low $\mathrm{pH}$ and High nitrate solutions have the highest $\mathrm{E}_{\text {corr }}$ values. This trend would be expected from solutions with greater halide concentrations. In general, the $\mathrm{E}_{\text {corr }}$ values do not change dramatically during the tests, as evidenced by how close most of the min imum $\mathrm{E}_{\mathrm{corr}}$ and maximum $\mathrm{E}_{\text {corr }}$ values are. Longer term tests would be needed, however, to determine if this trend continues. Test 071805 appears to have had problems likely rela ted to the reference electrode.

Table 3. Corrosion potential data (vs. SCE) collected prior to each electrochemical test.

\begin{tabular}{|c|c|c|c|c|c|c|c|}
\hline Test & Specimen & Solution & Temp & Initial $\mathrm{E}_{\text {corr }}$ & Final $E_{\text {corr }}$ & Min $E_{\text {corr }}$ & Max $E_{\text {corr }}$ \\
\hline 040405 & Cylinder & Average low $\mathrm{pH}$ & 60 & -0.3164 & -0.3364 & -0.3399 & -0.3164 \\
\hline 040605 & Cylinder & Average low $\mathrm{pH}$ & 60 & -0.3667 & -0.3565 & -0.3689 & -0.3550 \\
\hline 040805 & Cylinder & Average low $\mathrm{pH}$ & 60 & -0.3818 & -0.3654 & -0.3877 & -0.3640 \\
\hline 041205 & Cylinder & Average low $\mathrm{pH}$ & 60 & -0.3621 & -0.3883 & -0.3982 & -0.3577 \\
\hline 042205 & Cylinder & Average low $\mathrm{pH}$ & 60 & -0.3081 & -0.3427 & -0.3428 & -0.3081 \\
\hline 050305 & Cylinder & Average low $\mathrm{pH}$ & 60 & -0.3041 & -0.3224 & -0.3224 & -0.2984 \\
\hline 042505 & Cylinder & Average low $\mathrm{pH}$ & 90 & -0.3143 & -0.3255 & -0.3299 & -0.3138 \\
\hline 042605 & Cylinder & Average low $\mathrm{pH}$ & 90 & -0.3205 & -0.3086 & -0.3295 & -0.3066 \\
\hline 052405 & Cylinder & Average low $\mathrm{pH}$ & 90 & -0.3254 & -0.3303 & -0.3317 & -0.3225 \\
\hline 060205 & Cylinder & Average low $\mathrm{pH}$ & 90 & -0.3249 & -0.3354 & -0.3370 & -0.3241 \\
\hline 060105 & Crevice & Average low $\mathrm{pH}$ & 60 & -0.3260 & -0.3547 & -0.3567 & -0.3251 \\
\hline 060305 & Crevice & Average low $\mathrm{pH}$ & 60 & -0.3472 & -0.3617 & -0.3670 & -0.3468 \\
\hline 061305 & Crevice & Average low $\mathrm{pH}$ & 60 & -0.2932 & -0.3108 & -0.3114 & -0.2907 \\
\hline 051905 & Crevice & Average low $\mathrm{pH}$ & 90 & -0.3191 & -0.3204 & -0.3231 & -0.3172 \\
\hline 052005 & Crevice & Average low $\mathrm{pH}$ & 90 & -0.3429 & -0.3407 & -0.3479 & -0.3381 \\
\hline 062305 & Crevice & Average low $\mathrm{pH}$ & 90 & -0.3842 & -0.3664 & -0.3869 & -0.3645 \\
\hline 050505 & Cylinder & High nitrate & 60 & -0.3564 & -0.3632 & -0.3741 & -0.3527 \\
\hline 050605 & Cylinder & High nitrate & 60 & -0.3111 & -0.3361 & -0.3387 & -0.2979 \\
\hline 051605 & Cylinder & High nitrate & 60 & -0.3161 & -0.3457 & -0.3513 & -0.3160 \\
\hline
\end{tabular}


Table 3. (continued).

\begin{tabular}{|c|c|c|c|c|c|c|c|}
\hline Test & Specimen & Solution & Temp & Initial $E_{\text {corr }}$ & Final $E_{\text {corr }}$ & Min $E_{\text {corr }}$ & Max $E_{\text {corr }}$ \\
\hline 051705 & Cylinder & High nitrate & 90 & -0.2945 & -0.3066 & -0.3075 & -0.2918 \\
\hline 051805 & Cylinder & High nitrate & 90 & -0.3222 & -0.3233 & -0.3313 & -0.3193 \\
\hline 060805 & Crevice & High nitrate & 60 & -0.2867 & -0.3072 & -0.3097 & -0.2821 \\
\hline 081105 & Crevice & High nitrate & 60 & -0.3413 & -0.3445 & -0.3457 & -0.3343 \\
\hline 062105 & Crevice & High nitrate & 60 & -0.3351 & -0.3467 & -0.3477 & -0.3321 \\
\hline 070105 & Crevice & High nitrate & 90 & -0.3485 & -0.3202 & -0.3542 & -0.3183 \\
\hline 072705 & Crevice & High nitrate & 90 & -0.3623 & -0.3330 & -0.3658 & -0.3288 \\
\hline 072905 & Crevice & High nitrate & 90 & -0.3167 & -0.3006 & -0.3217 & -0.2972 \\
\hline 070605 & Crevice & High nitrate & 90 & -0.3143 & -0.3228 & -0.3231 & -0.3138 \\
\hline 071205 & Cylinder & High chloride & 60 & -0.3411 & -0.3499 & -0.3509 & -0.3398 \\
\hline 071505 & Cylinder & High chloride & 60 & -0.3415 & -0.3443 & -0.3451 & -0.3379 \\
\hline 071805 & Cylinder & High chloride & 90 & -0.3800 & -0.3465 & -0.5861 & -0.1038 \\
\hline 072005 & Cylinder & High chloride & 90 & -0.3651 & -0.3650 & -0.3669 & -0.3609 \\
\hline 072505 & Cylinder & High chloride & 90 & -0.3643 & -0.3648 & -0.3678 & -0.3610 \\
\hline 071405 & Crevice & High chloride & 60 & -0.3817 & -0.3790 & -0.3854 & -0.3745 \\
\hline 072105 & Crevice & High chloride & 60 & -0.4176 & -0.3997 & -0.4281 & -0.3997 \\
\hline 080105 & Crevice & High chloride & 60 & -0.3586 & -0.3628 & -0.3663 & -0.3502 \\
\hline 071905 & Crevice & High chloride & 90 & -0.4141 & -0.4039 & -0.4174 & -0.4019 \\
\hline 072605 & Crevice & High chloride & 90 & -0.3821 & -0.3656 & -0.3845 & -0.3626 \\
\hline 080205 & Crevice & High chloride & 90 & -0.3392 & -0.3533 & -0.3537 & -0.3370 \\
\hline 042705 & Cylinder & High halide & 60 & -0.4184 & -0.4179 & -0.4224 & -0.4173 \\
\hline 042805 & Cylinder & High halide & 60 & -0.4243 & -0.4267 & -0.4314 & -0.4236 \\
\hline 050905 & Cylinder & High halide & 60 & -0.4403 & -0.4487 & -0.4511 & -0.4403 \\
\hline 050205 & Cylinder & High halide & 90 & -0.4526 & -0.4377 & -0.4729 & -0.4376 \\
\hline 050405 & Cylinder & High halide & 90 & -0.5042 & -0.4503 & -0.5067 & -0.4500 \\
\hline 052505 & Cylinder & High halide & 90 & -0.4750 & -0.4388 & -0.4753 & -0.4386 \\
\hline 060605 & Crevice & High halide & 60 & -0.4095 & -0.4260 & -0.4265 & -0.4095 \\
\hline 060705 & Crevice & High halide & 60 & -0.4002 & -0.4202 & -0.4207 & -0.4000 \\
\hline 061405 & Crevice & High halide & 60 & -0.4205 & -0.4235 & -0.4314 & -0.4205 \\
\hline 080905 & Crevice & High halide & 90 & -0.4908 & -0.4567 & -0.4910 & -0.4561 \\
\hline 081005 & Crevice & High halide & 90 & -0.5070 & -0.4621 & -0.5103 & -0.4598 \\
\hline 063005 & Crevice & High halide & 90 & -0.4672 & -0.4409 & -0.4674 & -0.4407 \\
\hline
\end{tabular}




\subsection{Potentiodynamic Test Results}

\subsubsection{Explanation of Potentiodynamic Data}

The PD tests were used to determine the susceptibility to localized corrosion and determine the overall corrosion characteristics of the material in that environment. Cyclic PD polarization scans were employed to determine the passivation characteristics and specifically the repassivation potential $\left(\mathrm{E}_{\mathrm{rp}}\right)$. A scan includes both forward and reverse directions while a sweep is part of a scan performed in one direction (i.e., forward sweep).

Several parameters were obtained from these scans to allow comparison of the characteristics of each scan in numerical form. Figure 1 shows a diagram of a generic PD scan for the Ni-Cr-Mo-Gd alloy. The curves were split into two regions: (1) passive region and (2) transpassive region. The passive region begins at the initiation point of the PD curve to $0.6 \mathrm{~V}$. The designation of passive region does not necessarily designate the state of the material. The passive region is characterized as being stable under nonpitting environments. The transpassive region is from $0.6 \mathrm{~V}$ to the anodic limit $(0.8$ or $1.0 \mathrm{~V})$. This region is characterized as the point where uniform corrosion of the material is observed because of oxidation of $\mathrm{Cr}_{2} \mathrm{O}_{3}$ to chromate. Strictly speaking, choosing a value of $0.6 \mathrm{~V}$ to divide these two regions does not consider the different responses (shifts in the dividing point due to condition) but does provide a way to compare values under the various conditions examined. In both of these regions, the maximum current was determined (in forward or reverse sweeps). The maximum current in the passive region tends to drop after the initial forward sweep because of removal of the secondary phase, thus providing a measure of the degree of passivation for the material. The maximum current for the transpassive region always occurs at the anodic limit as would be expected. The maximum transpassive current tends to be similar in both scans as it is a uniform reaction for the base metal. Values for the charge measured in the passive and transpassive region were also obtained. In the passive region, forward and reverse sweep charge values were obtained separately. The charge in the transpassive region was the combination of the forward and reverse sweeps. As was observed for the maximum passive current, the initial forward passive charge is the greatest, whereas the subsequent charge values in the passive region decrease in value. This again provides a measure of the passivation characteristics of the material due to the removal of the gadolinide secondary phase.

The propensity for localized corrosion can also be ascertained by comparing the values of the corrosion potential $\left(\mathrm{E}_{\text {corr }}\right)$ and the critical potential $\left(\mathrm{E}_{\text {crit }}\right)$. No attempt was made to develop such a localized corrosion model. A value for the repassivation potential $\left(\mathrm{E}_{\mathrm{rp}}\right)$ was obtained for each PD scan. While several definitions of the repassivation potential can be used, the most common definition is the value where the reverse sweep (being higher than the forward sweep, i.e., positive hysteresis) crosses over (below) the value of the forward sweep. While this definition appears straightforward, in practice some level of interpretation is required. In some cases, the hysteresis (reverse sweep is positive of the forward sweep) is well negative of the anodic limit, and the curves may cross several times. The repassivation value is then obtained where the current for the reverse sweep crosses to lower current than the forward sweep for the final time. Part of the reason for this ambiguity and complexity may be due to the two-phase nature of this alloy as will be discussed in more detail below. 


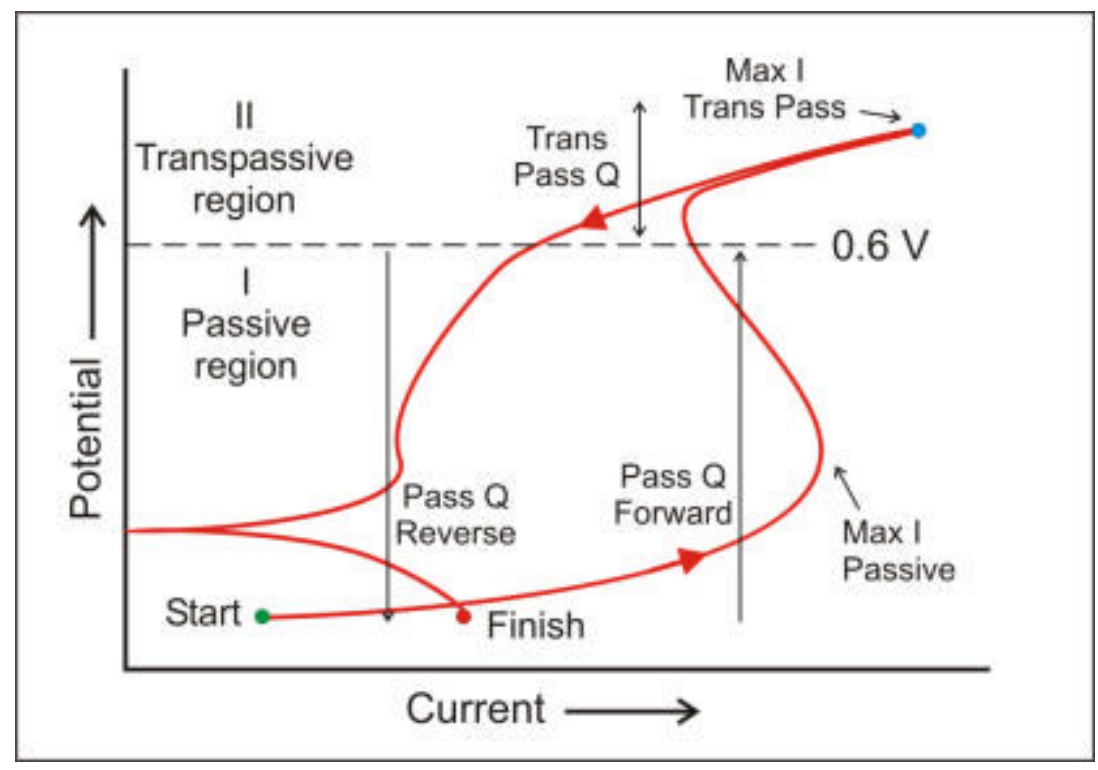

Figure 1. Diagram of a hypothetical potentiodynamic curve for Ni-Cr-Mo-Gd alloy demonstrating the various parameters collected from the curves.

\subsubsection{Potentiodynamic Tests Results}

The results of the PD tests are similar to what has been observed previously for Ni-Cr-Mo-Gd alloys (see Reference 8). Figure 2 shows two PD tests as examples of the type of behavior observed. The curves in Figure 2A for Test 042605 highlight the typical behavior for these alloys in benign environments (Average low $\mathrm{pH}$ solution at $60^{\circ} \mathrm{C}$ ). The initial forward sweep shows a broad current almost $1 \mathrm{~mA} / \mathrm{cm}^{2}$ in magnitude. This feature is primarily associated with the dissolution of the secondary phase. This was confirmed by LOM examination of the specimens, where small pits (5-20 Pm in diameter) are observed on the surface in a pattern consistent with the total removal of the surface exposed secondary phase. The reverse sweep response is also typical, where the current is about two orders of magnitude lower than that of the forward sweep. Hysteresis was not observed, partly because the current involved with dissolution of the gadolinide phase is so large. It is expected that most of the gadolinide phase has been removed by the completion of the forward sweep as the current actually drops significantly around $0.4 \mathrm{~V}$ before reaching the transpassive region. Similar behavior is also observed in the initial sweep in Figure 2B for Test 072605 , a crevice specimen in High halide solution at $90^{\circ} \mathrm{C}$ (a more aggressive condition). Interestingly, for Test 072605 , the current density is somewhat lower for the initial sweep and does not exhibit a smooth nature. This could be an effect of the crevice former obscuring the reaction to the internal parts of the crevice specimen resulting in poor mass transport and thus lower overall secondary phase dissolution rate at these inner locations. The level of passivation is also much less in the return sweep, which would also speak to a similar mass transport issue.

A second scan was also performed to demonstrate the properties of the material after removal of the secondary phase. Figure 2A shows that the forward sweep current is two orders of magnitude lower than that of the forward sweep of the initial scan. The reverse sweep shows a hysteresis from the transpassive region to approximately $0.4 \mathrm{~V}(0.3870 \mathrm{~V})$, the repassivation potential. Similar behavior is observed for the crevice specimen in Figure 2B with almost an order of magnitude more current, not unexpected due to the more aggressive conditions. The $\mathrm{E}_{\mathrm{rp}}$ occurs around $0.1 \mathrm{~V}(0.1371 \mathrm{~V})$. Crevice corrosion is quite likely although extensive damage was not observed under the crevice former. The limited time the specimen existed in the critical condition may prevent extensive damage from occurring. 

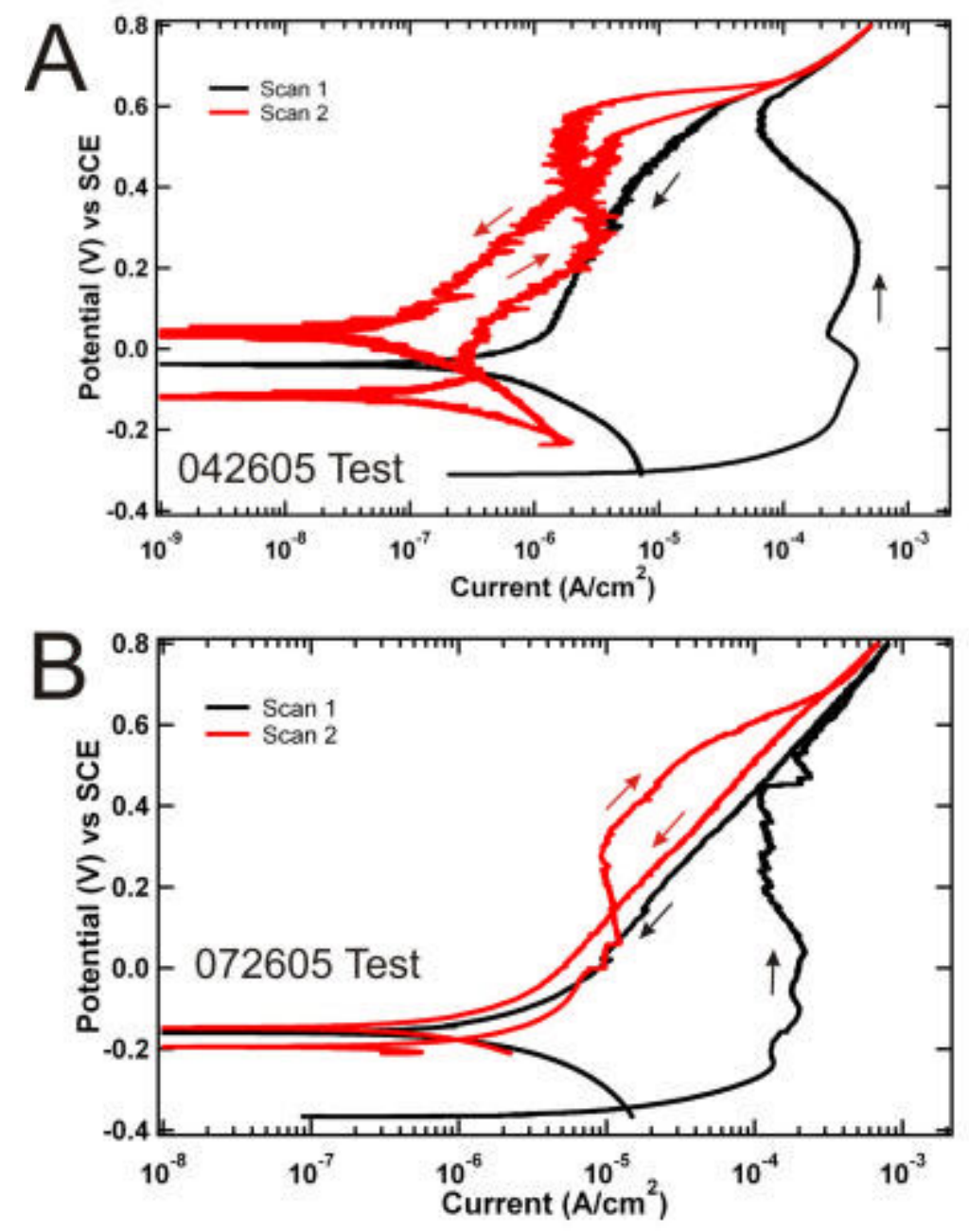

Figure 2. Potentiodynamic curves for Tests 042605 and 072605 . See Tables 4 and 5 for conditions.

The repassivation potentials $\left(\mathrm{E}_{\mathrm{rp}}\right)$ were evaluated using the crossover criterion (where reverse sweep last crosses the forward sweep). Tables 4 and 5 provide these values for the cylindrical and crevice specimens, respectively. All tests are included in these tables, and some anomalies are noted in the EC Comments column. In general, repassivation values were not observed in the initial scan because of the large current for gadolinide dissolution in the forward sweep as discussed earlier. The exception to this is found in the High halide solution where hysteresis was observed for $90^{\circ} \mathrm{C}$ tests for both types of specimens. This test condition had a relatively low current in the initial forward sweep but also had very low degree of repassivation in the subsequent sweeps. Repassivation potentials were observed in most of the second scans and generally were between 0.2 to $-0.2 \mathrm{~V}$. However, in some cases the repassivation value is much more positive. Much of the discrepancy occurs as the currents for the forward and reverse scans are very similar and often cross over several times. A current magnitude-based criterion should be considered as an alternative value in future work or in further analysis of this data.

Other parameters in Tables 4 and 5 can be examined to show the trends mentioned earlier. In all tests, the passive charge in the forward sweep for the initial scan is greater than the reverse sweep (and also subsequent sweeps as well). The forward and reverse sweep charge tended to be similar for the second scan, as the base material was controlling the corrosion properties of the material in both 
directions. The maximum current in the passive range for the first scan was in almost all cases greater than the value for the second scan. The transpassive parameters are similar in value for both scans.

The transpassive parameters (maximum current and charge) tended to increase with temperature. In tests where the anodic limit was $1.0 \mathrm{~V}$, the solutions displayed a yellow color indicative of significant $\mathrm{Cr}_{2} \mathrm{O}_{3}$ oxidation. The anodic limit was moved negative $(0.8 \mathrm{~V})$ after the initial tests to avoid excessive transpassive dissolution. The specimens had some faint coloration probably due to transpassive dissolution products. This was removed during the post-test descaling in many cases. 


\begin{tabular}{|c|c|c|c|c|c|c|c|c|c|c|c|c|c|c|c|c|c|c|c|}
\hline 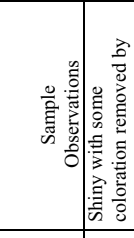 & 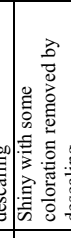 & & & & & & & & & & 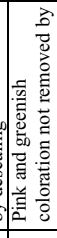 & 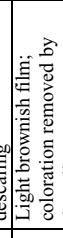 & & 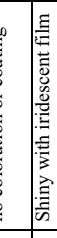 & & $\overline{\underline{v}}$ & & 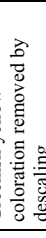 & 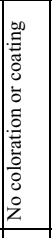 \\
\hline 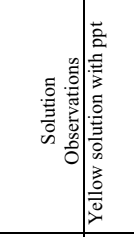 & 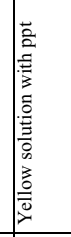 & 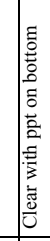 & 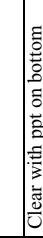 & 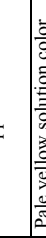 & 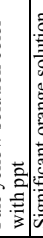 & & & & & 竎 & 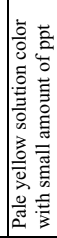 & 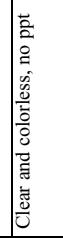 & & 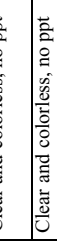 & & & & & 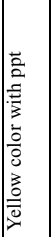 \\
\hline 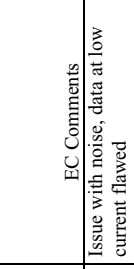 & 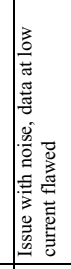 & 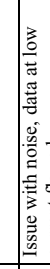 & 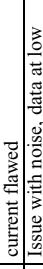 & & & 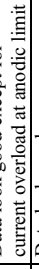 & & & & 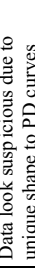 & 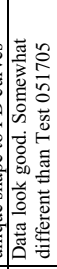 & 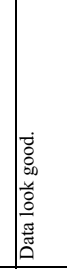 & & 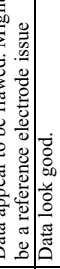 & 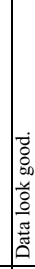 & & & & 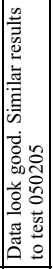 \\
\hline $\begin{array}{l}\infty \\
\vdots \\
\vdots \\
0 \\
0\end{array}$ & $\begin{array}{l}0 \\
\vdots \\
0 \\
0 \\
0\end{array}$ & 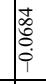 & $\begin{array}{l}n \\
0 \\
0 \\
0 \\
0 \\
0\end{array}$ & : & 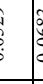 & & 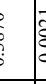 & & & 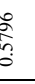 & $\begin{array}{l}0 \\
\infty \\
0 \\
0 \\
0 \\
\end{array}$ & 总 & & 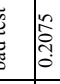 & $\infty$ & 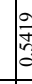 & 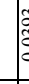 & & \begin{tabular}{|l}
0 \\
0 \\
0 \\
0 \\
0 \\
0
\end{tabular} \\
\hline 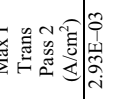 & 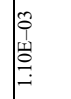 & 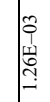 & 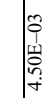 & 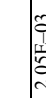 & 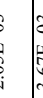 & & 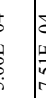 & & 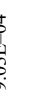 & 管 & 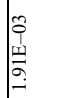 & 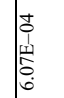 & & 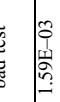 & 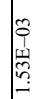 & $\frac{1}{b}$ & 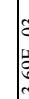 & & 孚 \\
\hline & สิ & $\underset{\substack{f \\
\text { i }}}{ }$ & $\tilde{n}$ & $\sigma^{\circ}$ & 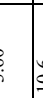 & 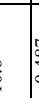 & 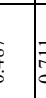 & & 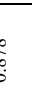 & 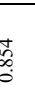 & 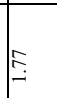 & 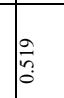 & & $\underset{s}{s}$ & 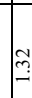 & 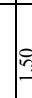 & $\xi$ & & के \\
\hline 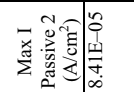 & 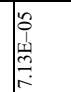 & 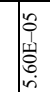 & 焉 & 童 & 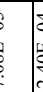 & 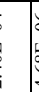 & 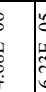 & & 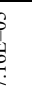 & 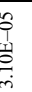 & 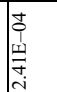 & 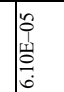 & & 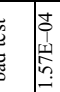 & 离 & in & 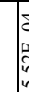 & & 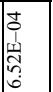 \\
\hline 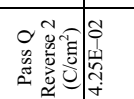 & 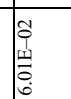 & 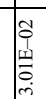 & 离 & 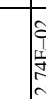 & 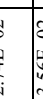 & 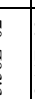 & 8 & & 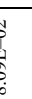 & 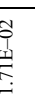 & 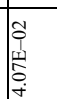 & 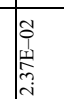 & & 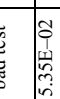 & 苟 & 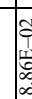 & 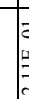 & & \begin{tabular}{|l|}
$\overrightarrow{0}$ \\
产 \\
in
\end{tabular} \\
\hline 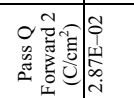 & 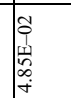 & 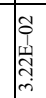 & 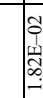 & $\frac{\AA}{d}$ & 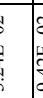 & 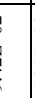 & 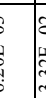 & & 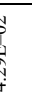 & 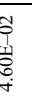 & 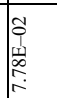 & 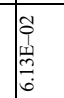 & & 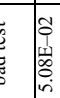 & 豆 & $\overline{9}$ & 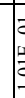 & & 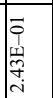 \\
\hline & $\frac{0}{2}$ & 受 & 产 & 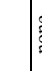 & 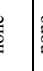 & 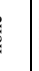 & $=$ & & 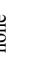 & 产 & 产 & 受 & & 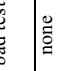 & 产 & 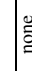 & 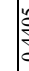 & 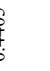 & 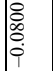 \\
\hline 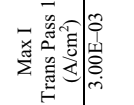 & 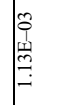 & 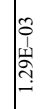 & 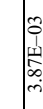 & 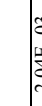 & 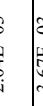 & 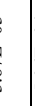 & 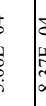 & & 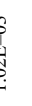 & 惫 & 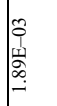 & 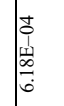 & & 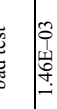 & 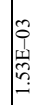 & $\begin{array}{l}0 \\
0 \\
\frac{1}{6}\end{array}$ & & & 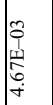 \\
\hline & ì & रे & $\approx$ & 8 & 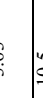 & 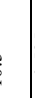 & 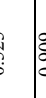 & & & 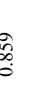 & $\stackrel{9}{=}$ & $\mid \begin{array}{l}0 \\
0 \\
0 \\
0 \\
0\end{array}$ & & 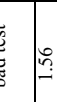 & & 5 & & 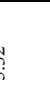 & 多 \\
\hline 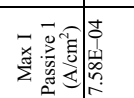 & 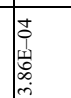 & 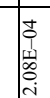 & 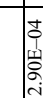 & 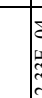 & 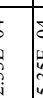 & 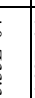 & 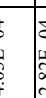 & & 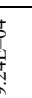 & 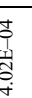 & 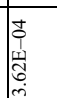 & 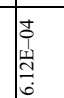 & & 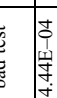 & $\stackrel{t}{*}$ & 辛 & 9 & & 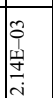 \\
\hline 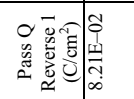 & 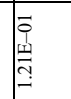 & 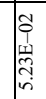 & 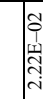 & 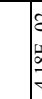 & 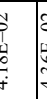 & 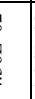 & 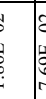 & & 5 & 离 & 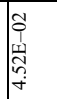 & 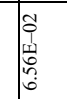 & & 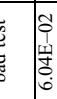 & $\bar{E}$ & 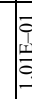 & & 5 & 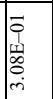 \\
\hline 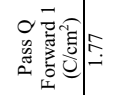 & $\because$ & हีं & : & 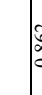 & 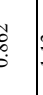 & & 5 & & & 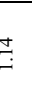 & $\cong$ & $\vec{i}$ & & 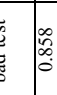 & & & & 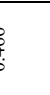 & 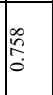 \\
\hline 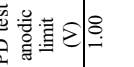 & 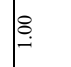 & 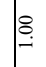 & 8 & 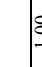 & 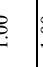 & & 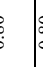 & & & 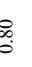 & 定 & 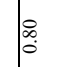 & & $\stackrel{\square}{:}$ & & & & 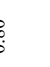 & 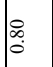 \\
\hline 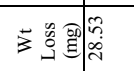 & $\begin{array}{l}2 \\
\dot{q} \\
\dot{q}\end{array}$ & $\stackrel{m}{m}$ & 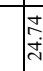 & 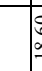 & : & & & & & & $\hat{E}$ & $\begin{array}{l}\infty \\
0 \\
0 \\
0\end{array}$ & j & 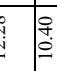 & & & & f & $\frac{\pi}{\pi j}$ \\
\hline 言迥造 & 8 & 8 & 8 & 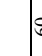 & 8 & & $?$ & & & & 8 & 8 & & 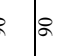 & & & & 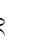 & 2 \\
\hline 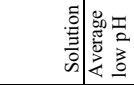 & 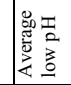 & & & & & & & & & & $E$ & 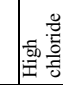 & & & & & & 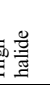 & 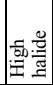 \\
\hline 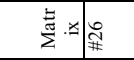 & $\dddot{\sharp}$ & $¥$ & \% & 19 & 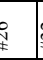 & & 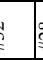 & & 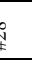 & $\frac{7}{7 f}$ & $\begin{array}{l} \pm \\
¥ \\
¥\end{array}$ & 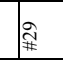 & 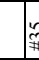 & $\tilde{m}$ & | $P$ & 舟 & $\approx$ & 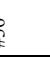 & $\%$ \\
\hline 空谇 & 等 & 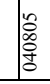 & 商 & 18 & 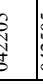 & & 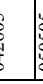 & & & $\begin{array}{l}2 \\
\frac{2}{2} \\
\frac{a}{2} \\
0\end{array}$ & 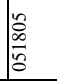 & 商 & 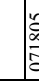 & $\begin{array}{l}0 \\
0 \\
0 \\
0 \\
0 \\
0\end{array}$ & & & & & 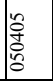 \\
\hline
\end{tabular}




\begin{tabular}{|c|c|c|c|c|c|c|c|c|c|c|c|c|c|c|c|c|}
\hline & 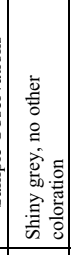 & 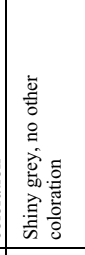 & 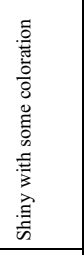 & 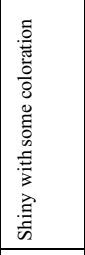 & 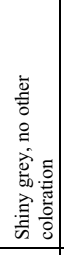 & 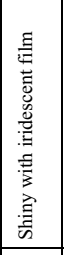 & 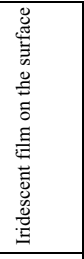 & 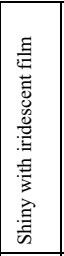 & 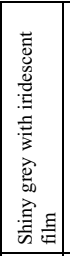 & 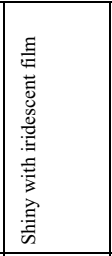 & 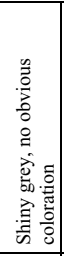 & 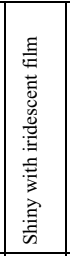 & 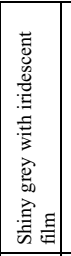 & 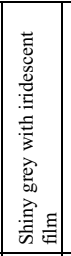 & 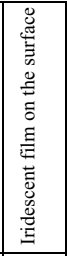 & 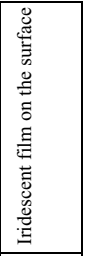 \\
\hline & 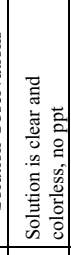 & 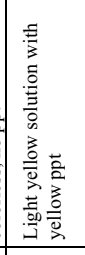 & 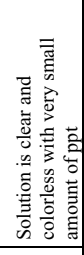 & 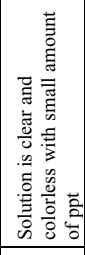 & 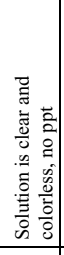 & 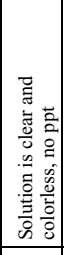 & 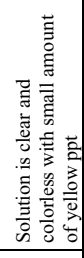 & 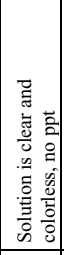 & 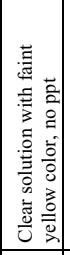 & 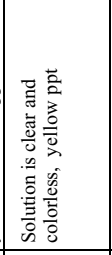 & 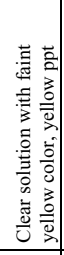 & 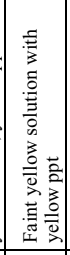 & 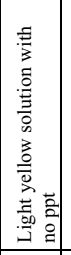 & 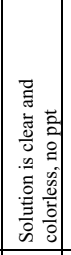 & 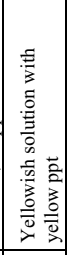 & 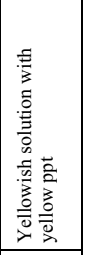 \\
\hline & 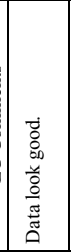 & 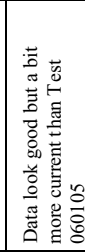 & 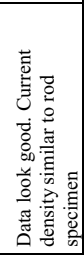 & 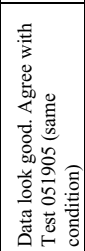 & 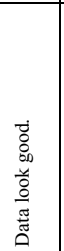 & 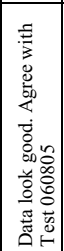 & 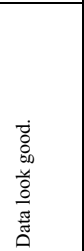 & 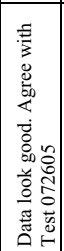 & 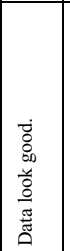 & 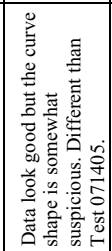 & 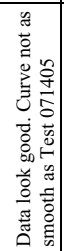 & 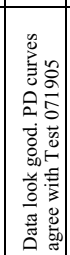 & 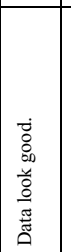 & 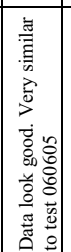 & 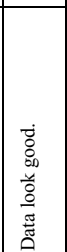 & 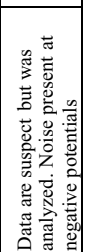 \\
\hline : 8 & $=\frac{\frac{0}{n}}{0}$ & 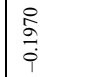 & 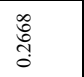 & \begin{tabular}{|l|} 
\\
\\
$\frac{1}{2}$ \\
0
\end{tabular} & 总 & 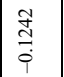 & 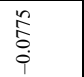 & 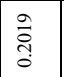 & 总 & \begin{tabular}{|l|l}
0 \\
0 \\
0 \\
0 \\
0
\end{tabular} & \begin{tabular}{|l|}
$\frac{n}{0}$ \\
$\frac{0}{0}$
\end{tabular} & $\begin{array}{l}\sqrt{2} \\
\stackrel{9}{0}\end{array}$ & 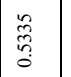 & 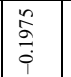 & 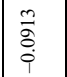 & 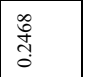 \\
\hline 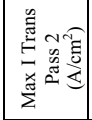 & 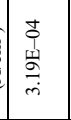 & 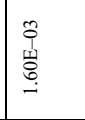 & 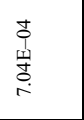 & 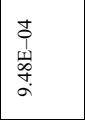 & 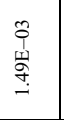 & 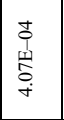 & 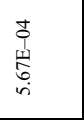 & 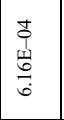 & $\begin{array}{l}+ \\
0 \\
\text { 岁 } \\
0\end{array}$ & \begin{tabular}{|l} 
\\
0 \\
票 \\
d
\end{tabular} & 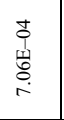 & 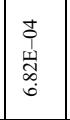 & 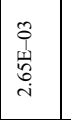 & 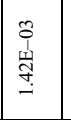 & 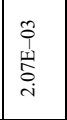 & 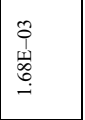 \\
\hline 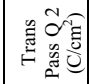 & ते & $\overrightarrow{\underline{n}}$ & $\begin{array}{l}\approx \\
\infty \\
\infty \\
\infty\end{array}$ & $\begin{array}{l}\text { oे } \\
\text { o. }\end{array}$ & 商 & 童 & $\begin{array}{l}\text { 我 } \\
\vdots \\
0\end{array}$ & : & 总 & \begin{tabular}{|l} 
索 \\
|
\end{tabular} & శ్ & $\begin{array}{l}\infty \\
\infty \\
\infty \\
\infty\end{array}$ & $\stackrel{\stackrel{6}{i}}{\mathrm{i}}$ & Iี & $\underset{i}{i}$ & $\underbrace{\infty}_{-}$ \\
\hline 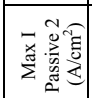 & 总 & 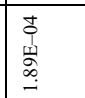 & 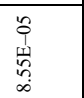 & 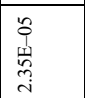 & 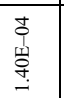 & \begin{tabular}{|l|}
\multirow{2}{*}{} \\
湈 \\
g.
\end{tabular} & 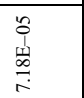 & 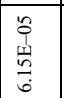 & 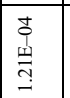 & 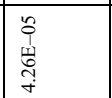 & 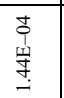 & 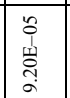 & 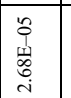 & 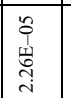 & 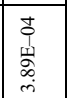 & 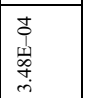 \\
\hline 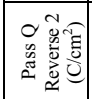 & 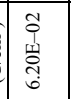 & 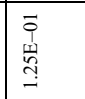 & \begin{tabular}{|l|}
\multirow{8}{*}{} \\
岕 \\
in \\
\end{tabular} & 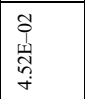 & 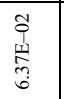 & 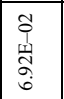 & 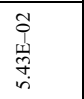 & 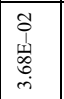 & 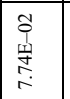 & \begin{tabular}{|l|}
\multirow{3}{*}{} \\
容 \\
0
\end{tabular} & 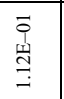 & 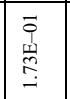 & 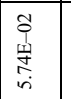 & 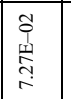 & 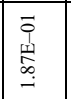 & \begin{tabular}{|l|}
$\overline{9}$ \\
岁 \\
㝵 \\
-
\end{tabular} \\
\hline 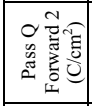 & 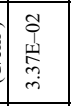 & 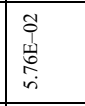 & 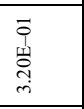 & 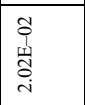 & 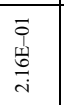 & 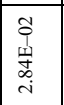 & 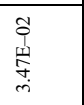 & 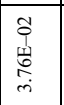 & 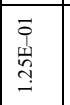 & 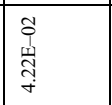 & 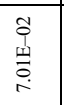 & 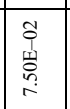 & 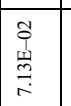 & 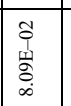 & $\begin{array}{l}\overrightarrow{0} \\
\text { 离 } \\
\stackrel{5}{\circ} \\
\end{array}$ & 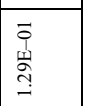 \\
\hline 룔ㄹ & $=\stackrel{\circ}{\stackrel{\circ}{E}}$ & 产 & 兽 & 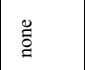 & 总 & \begin{tabular}{|l|l}
\multirow{g}{g}{} \\
\multirow{g}{*}{}
\end{tabular} & 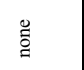 & \begin{tabular}{|l|}
$\frac{9}{g}$ \\
$g$
\end{tabular} & 总 & \begin{tabular}{|l}
0 \\
\end{tabular} & 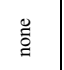 & 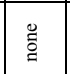 & $\mid \stackrel{\circ}{\stackrel{\circ}{\Xi}}$ & 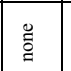 & 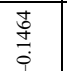 & 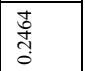 \\
\hline 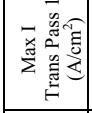 & 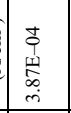 & 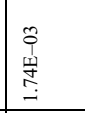 & 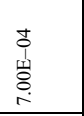 & 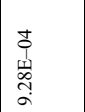 & 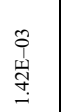 & 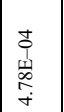 & $\begin{array}{l}\text { ta } \\
\text { 岁 } \\
\text { s. } \\
\text { n. }\end{array}$ & 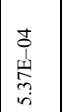 & 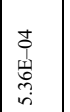 & 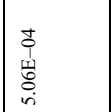 & 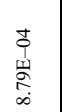 & 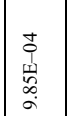 & 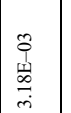 & 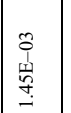 & 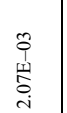 & 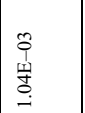 \\
\hline 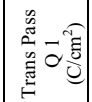 & 勇 & 罗 & 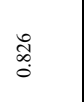 & $\stackrel{\Xi}{\Xi}$ & $\Xi$ & 管 & 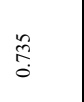 & 总 & 或 & 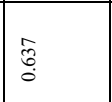 & 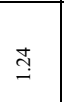 & ఫ̊ & $\tilde{\tilde{~}}$ & $\overrightarrow{3}$ & $\Rightarrow$ & 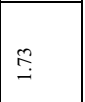 \\
\hline 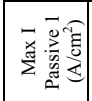 & 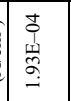 & $\begin{array}{l}\overrightarrow{0} \\
\stackrel{1}{2} \\
\text { مे }\end{array}$ & 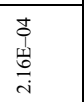 & 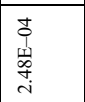 & 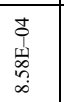 & 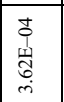 & 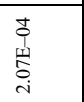 & 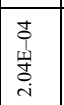 & 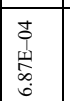 & 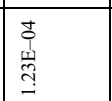 & 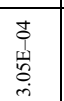 & 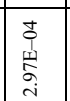 & 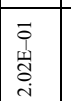 & 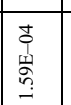 & 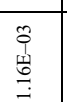 & 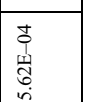 \\
\hline 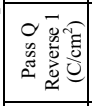 & 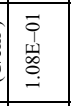 & \begin{tabular}{|l|}
$\overrightarrow{0}$ \\
岁 \\
- \\
\end{tabular} & 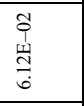 & 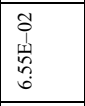 & 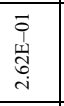 & 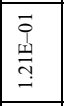 & 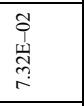 & 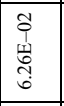 & \begin{tabular}{|c|}
$\overrightarrow{0}$ \\
$\omega$ \\
\\
$i$ \\
\end{tabular} & \begin{tabular}{|l|}
$\overrightarrow{0}$ \\
山े \\
$\stackrel{9}{-}$ \\
\end{tabular} & \begin{tabular}{|l|}
$\overline{0}$ \\
$\omega$ \\
$\infty$ \\
0 \\
$n$ \\
$n$
\end{tabular} & 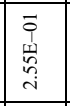 & 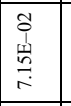 & 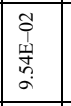 & 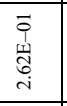 & \begin{tabular}{|l|l}
$\overrightarrow{5}$ \\
亲 \\
in \\
\end{tabular} \\
\hline 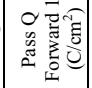 & 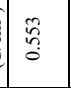 & 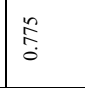 & $\vec{\sigma}$ & $\mid \begin{array}{l}\tilde{n} \\
\stackrel{2}{0} \\
0\end{array}$ & $\stackrel{\infty}{3}$ & 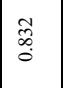 & 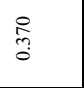 & : & a & है. & $\begin{array}{l}\vec{n} \\
0\end{array}$ & \begin{tabular}{|c|c}
$\vec{\infty}$ \\
$\stackrel{\infty}{\infty}$
\end{tabular} & 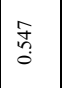 & 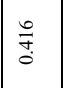 & 莒 & gี \\
\hline 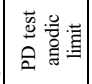 & $\stackrel{\infty}{\infty}$ & $\stackrel{\infty}{\circ}$ & $\stackrel{\infty}{\infty}$ & $\stackrel{\infty}{\infty}$ & $\stackrel{\infty}{\infty}$ & $\stackrel{\infty}{\circ}$ & $\stackrel{\infty}{\infty}$ & $\infty$ & $\stackrel{\infty}{\circ}$ & $\stackrel{\infty}{\circ}$ & $\stackrel{\infty}{\infty}$ & $\stackrel{\infty}{\circ}$ & $\stackrel{\infty}{\infty}$ & \begin{tabular}{|l}
$\infty$ \\
$\infty$ \\
$\infty$
\end{tabular} & $\stackrel{\infty}{\infty}$ & $\stackrel{\infty}{\infty}$ \\
\hline 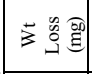 & $\frac{\infty}{\infty}$ & $\begin{array}{l}\stackrel{尺}{\infty} \\
\stackrel{\infty}{q}\end{array}$ & $\stackrel{\circ}{\underline{I}}$ & 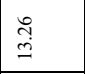 & 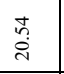 & \begin{tabular}{|l}
\multirow{2}{*}{} \\
\end{tabular} & $\overrightarrow{\sigma^{\prime}}$ & 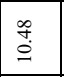 & 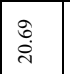 & 号 & $\begin{array}{l} \\
\text { 寺 }\end{array}$ & $\begin{array}{l}\infty \\
\stackrel{\infty}{=}\end{array}$ & 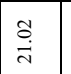 & 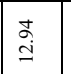 & 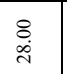 & 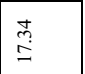 \\
\hline 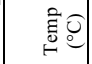 & 8 & 8 & 8 & : & 8 & 8 & 8 & 8 & 8 & 8 & 8 & 8 & 8 & 8 & 8 & 8 \\
\hline & & 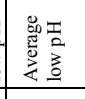 & 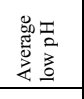 & 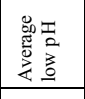 & 总尊 & 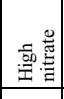 & 总总 & 王 & 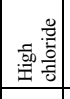 & 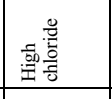 & 总部 & 离部 & 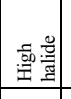 & 总: & 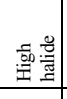 & 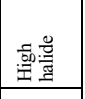 \\
\hline$\frac{\pi}{2}$ & 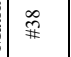 & $\mid$\begin{tabular}{c}
$\infty$ \\
\hdashline \\
\hdashline
\end{tabular} & 素 & 素 & 薏 & 暴 & 柿 & 释 & 䒠 & 素 & 惄 & 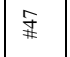 & 素 & 买 & 斑 & 喿 \\
\hline 旁= & $\begin{array}{l}\text { \%о } \\
\text { : } \\
0\end{array}$ & 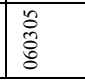 & 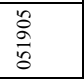 & 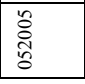 & 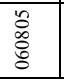 & 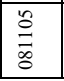 & 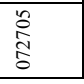 & 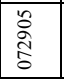 & \begin{tabular}{|l|l}
$\frac{8}{0}$ \\
$\frac{8}{8}$ \\
\end{tabular} & 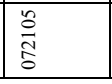 & 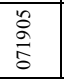 & 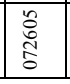 & 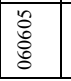 & 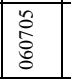 & 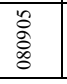 & 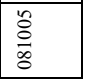 \\
\hline
\end{tabular}




\subsection{Linear Polarization Resistance Measurements}

LPR measurements were performed for PD tests following the $\mathrm{E}_{\text {corr }}$ measurement. The measurement was used to determine the corrosion rate of the alloy. It has been established in previous work that the secondary phase is much more susceptible to corrosion than the base material and is subject to selective dissolution processes (see References 4 through 8). During the LPR tests, the secondary phase is likely intact during the measurement. Thus the corrosion rates are expected to be controlled by the corrosion rate of the gadolinide phase. The corrosion rates by this method are much higher than what is obtained from PS tests as will be shown below.

Table 6 shows the LPR-determined corrosion rates for the 34 LPR tests performed. The corrosion rates covered a large range as high as $506 \mathrm{Pm} / \mathrm{yr}$ and as low as $2.1 \mathrm{Pm} / \mathrm{yr}$ with much scatter. From examination of the data performed under similar conditions, the corrosion rates were not very reproducible. The average corrosion rate for all the tests was $211 \pm 161 \mathrm{Pm} / \mathrm{yr}$ (not including Test 081005 value). There was not a clear trend toward higher corrosion rates with increasing temperature or with the type of specimen. Also, these values were obtained from generic values of the Stern-Geary coefficient. Thus, these values are more useful for comparison purposes within this data set or for similar alloys under similar conditions.

Table 6. LPR derived corrosion rates.

\begin{tabular}{|c|c|c|c|c|c|}
\hline Test ID & $\begin{array}{l}\text { Specimen } \\
\text { type }\end{array}$ & Matrix & Solution & $\begin{array}{l}\text { Temp } \\
\left({ }^{\circ} \mathrm{C}\right)\end{array}$ & $\begin{array}{l}\text { LPR CR } \\
(\mathrm{Pm} / \mathrm{yr})\end{array}$ \\
\hline 040405 & Cylinder & $\# 26$ & $\begin{array}{l}\text { Average low } \\
\text { pH }\end{array}$ & 60 & 506 \\
\hline 040605 & Cylinder & \#26 & $\begin{array}{l}\text { Average low } \\
\mathrm{pH}\end{array}$ & 60 & 97.3 \\
\hline 040805 & Cylinder & $\# 26$ & $\begin{array}{l}\text { Average low } \\
\mathrm{pH}\end{array}$ & 60 & 46.9 \\
\hline 041205 & Cylinder & $\# 26$ & $\begin{array}{l}\text { Average low } \\
\mathrm{pH}\end{array}$ & 60 & 43.2 \\
\hline 042205 & Cylinder & $\# 26$ & $\begin{array}{l}\text { Average low } \\
\mathrm{pH}\end{array}$ & 60 & 369 \\
\hline 042505 & Cylinder & $\# 32$ & $\begin{array}{l}\text { Average low } \\
\mathrm{pH}\end{array}$ & 90 & 490 \\
\hline 042605 & Cylinder & $\# 32$ & $\begin{array}{l}\text { Average low } \\
\mathrm{pH}\end{array}$ & 90 & 308 \\
\hline 060105 & Crevice & \#38 & $\begin{array}{l}\text { Average low } \\
\mathrm{pH}\end{array}$ & 60 & 211 \\
\hline 060305 & Crevice & $\# 38$ & $\begin{array}{l}\text { Average low } \\
\mathrm{pH}\end{array}$ & 60 & 137 \\
\hline 051905 & Crevice & $\# 44$ & $\begin{array}{l}\text { Average low } \\
\mathrm{pH}\end{array}$ & 90 & 278 \\
\hline 052005 & Crevice & $\# 44$ & $\begin{array}{l}\text { Average low } \\
\mathrm{pH}\end{array}$ & 90 & 329 \\
\hline 050505 & Cylinder & $\# 28$ & High nitrate & 60 & 139 \\
\hline 050605 & Cylinder & $\# 28$ & High nitrate & 60 & 410 \\
\hline
\end{tabular}


Table 6. (continued).

\begin{tabular}{|c|c|c|c|c|c|}
\hline Test ID & $\begin{array}{c}\text { Specimen } \\
\text { type }\end{array}$ & Matrix & Solution & $\begin{array}{c}\text { Temp } \\
\left({ }^{\circ} \mathrm{C}\right)\end{array}$ & $\begin{array}{c}\text { LPR CR } \\
(\mathrm{Pm} / \mathrm{yr})\end{array}$ \\
\hline 051705 & Cylinder & $\# 34$ & High nitrate & 90 & 452 \\
\hline 051805 & Cylinder & $\# 34$ & High nitrate & 90 & 297 \\
\hline 081105 & Crevice & $\# 40$ & High nitrate & 60 & 169 \\
\hline 060805 & Crevice & $\# 40$ & High nitrate & 60 & 553 \\
\hline 072705 & Crevice & $\# 46$ & High nitrate & 90 & 88.6 \\
\hline 072905 & Crevice & $\# 46$ & High nitrate & 90 & 237 \\
\hline 071205 & Cylinder & $\# 29$ & High chloride & 60 & 385 \\
\hline 071805 & Cylinder & $\# 35$ & High chloride & 90 & 326 \\
\hline 072005 & Cylinder & $\# 35$ & High chloride & 90 & 314 \\
\hline 071405 & Crevice & $\# 41$ & High chloride & 60 & 78.6 \\
\hline 072105 & Crevice & $\# 41$ & High chloride & 60 & 6.89 \\
\hline 071905 & Crevice & $\# 47$ & High chloride & 90 & 68.3 \\
\hline 072605 & Crevice & $\# 47$ & High chloride & 90 & 153 \\
\hline 042705 & Cylinder & $\# 30$ & High halide & 60 & 143 \\
\hline 042805 & Cylinder & $\# 30$ & High halide & 60 & 120 \\
\hline 050205 & Cylinder & $\# 36$ & High halide & 90 & 75.7 \\
\hline 050405 & Cylinder & $\# 36$ & High halide & 90 & 28.4 \\
\hline 060605 & Crevice & $\# 42$ & High halide & 60 & 50.9 \\
\hline 060705 & Crevice & $\# 42$ & High halide & 60 & 60.2 \\
\hline 080905 & Crevice & $\# 48$ & High halide & 90 & 2.1 \\
\hline 081005 & Crevice & $\# 48$ & High halide & 90 & $0.698^{*}$ \\
\hline$*$ Test ID 081005 appears to be flawed. & & & & \\
\hline
\end{tabular}

\subsection{Potentiostatic Test Results}

\subsubsection{Explanation of Potentiostatic Tests}

PS tests were also used to evaluate the corrosion properties of the alloy. In this case, the specimen is held at one potential while measuring the current flow. For a passive metal, it would be expected that the current would drop during the test as the passive oxide film thickens and composition changes. Under conditions where the material might be susceptible to localized corrosion, the current could increase, possibly in a chaotic fashion because of pit evolution processes. The potential used in the test should be representative of what might be expected for the $\mathrm{E}_{\text {corr }}$ value or some type of boundary condition (more positive than expected). As the pitting probability usually increases with potential, a bounding case would be to use a more positive value than the expected $\mathrm{E}_{\text {corr }}$. In addition, a value for the corrosion rate at the potential chosen can be calculated from the corrosion current $\left(\mathrm{I}_{\text {corr }}\right)$. The value chosen in these tests, $+0.2 \mathrm{~V}$, is likely a bounding $\mathrm{E}_{\text {corr }}$ value and thus should represent a bounding case for determining the corrosion rate from $\mathrm{I}_{\text {corr }}$.

In these tests, the expected long-term $\mathrm{E}_{\text {corr }}$ value was not known. A value of $+0.2 \mathrm{~V}$ is known from previous testing of this alloy to remove the secondary gadolinide phase (under different conditions) exposed to the surface, leaving a more passive surface than the initial condition. The PS tests highlight the 
tendency of the material to become more passive over time. The tests were performed for a period of 7 days. The maximum current and the total charge during the test are also reported. A second corrosion rate based on specimen weight loss was also calculated using a method for exposure coupons.

\subsubsection{Potentiostatic Test Results}

Tables 7 and 8 contain the data obtained from the PS tests for cylindrical and crevice specimens, respectively. Two curves for two extremes of behavior are shown in Figure 3. This figure displays the tendency for the current to decrease during the experiment. The curve for the cylindrical specimen Test 072505 shows the current drops to approximately $30 \mathrm{nA} / \mathrm{cm}^{2}$, while the crevice specimen Test 063005 for the same condition shows a final current of approximately $3 \mathrm{PA} / \mathrm{cm}^{2}$, two orders of magnitude higher. The difference in these two tests is due to crevice attack of the base material for Test 063005, as evidenced from observations of attack at the crevice former following the test. Passivation was observed with corrosion rates less than approximately $2 \mathrm{Pm} / \mathrm{yr}$ (calculated from $\mathrm{I}_{\text {corr }}$ ) for all tests except the crevice specimens in the High halide and High chloride solutions. All crevice tests in these two solutions indicated crevice attack and much higher corrosion rates as calculated from the $\mathrm{I}_{\text {corr }}$. The observed crevice damage, corrosion rates, and weight loss increased significantly with temperature in these solutions.

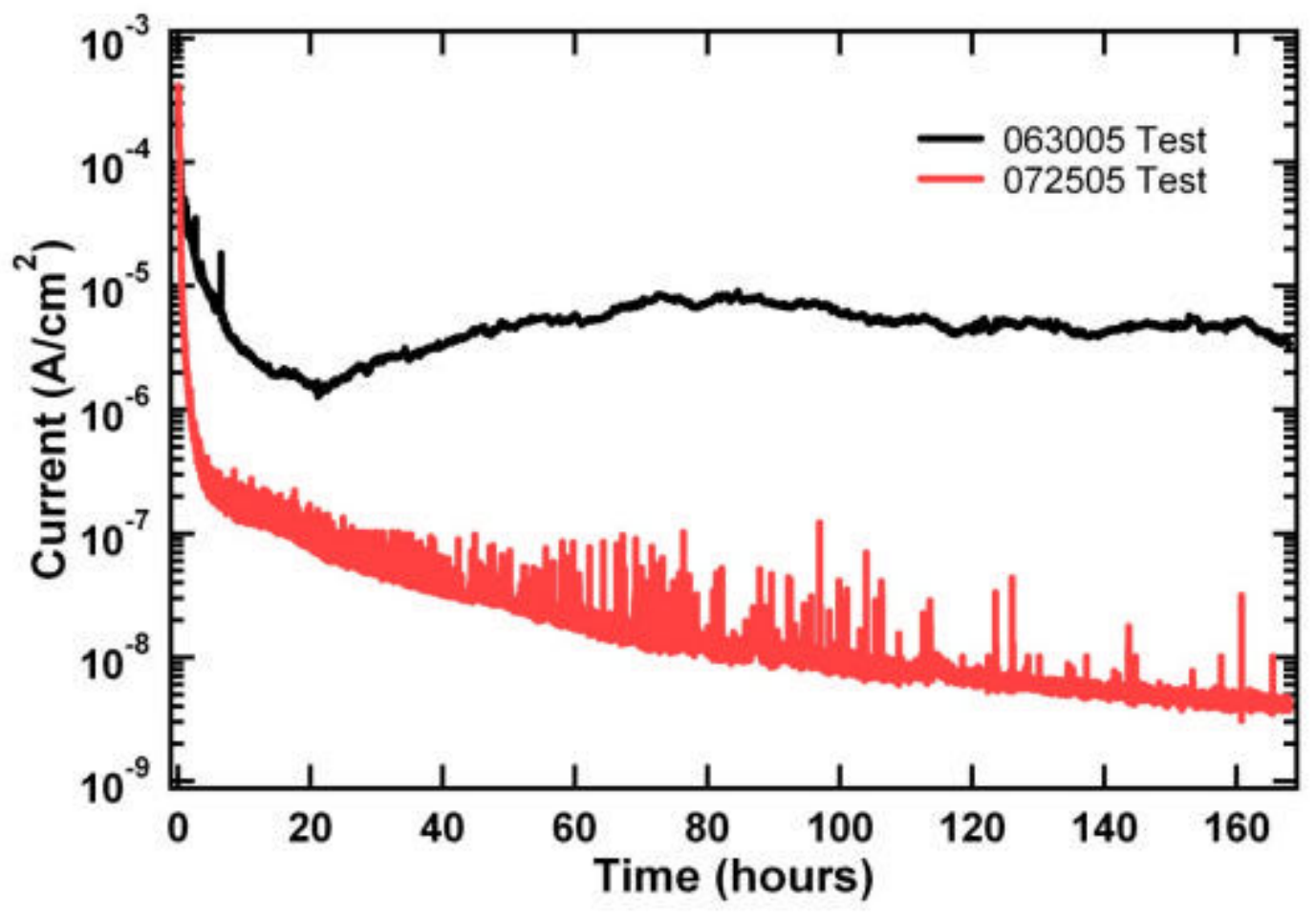

Figure 3. Potentiostatic curves for Tests 063005 and 072605 . See Tables 7 and 8 for conditions. 


\begin{tabular}{|c|c|c|c|c|c|c|c|c|}
\hline 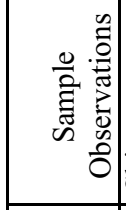 & 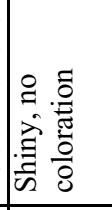 & 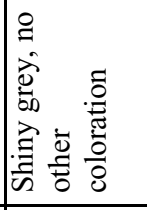 & 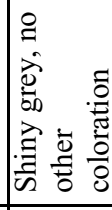 & 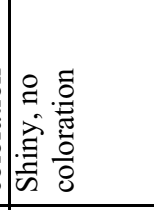 & 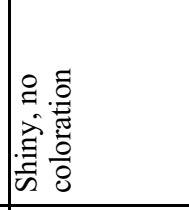 & 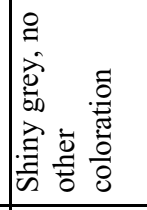 & 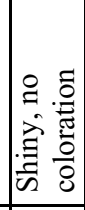 & 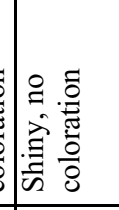 \\
\hline 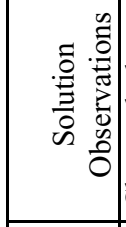 & 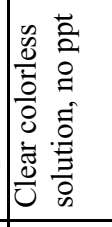 & 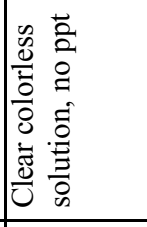 & 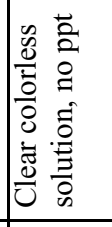 & 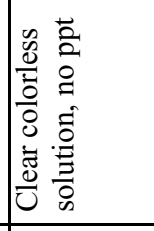 & 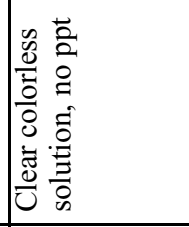 & 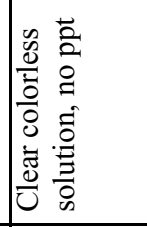 & 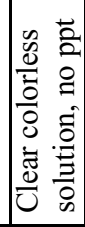 & 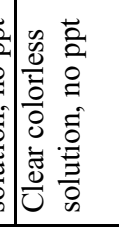 \\
\hline 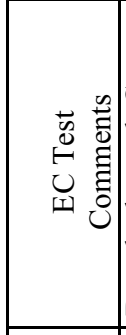 & 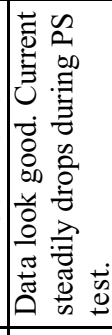 & 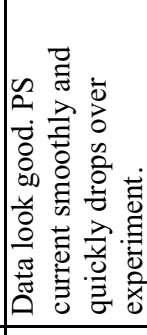 & 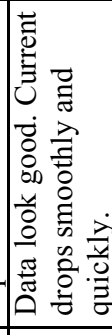 & 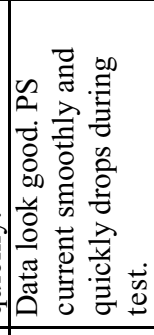 & 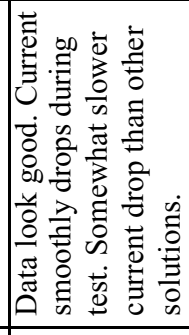 & 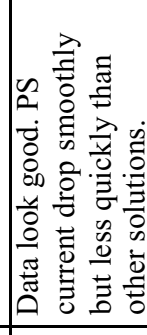 & 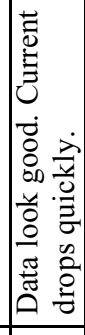 & 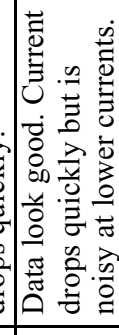 \\
\hline 尽 & 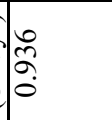 & $\stackrel{n}{a}$ & $\hat{\underline{s}}$ & $\stackrel{-}{\mathrm{A}}$ & $\stackrel{n}{\dddot{m}}$ & : & $\stackrel{8}{\circ}$ & bे \\
\hline 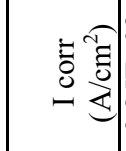 & 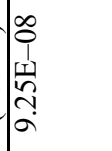 & 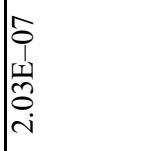 & $\begin{array}{l}\hat{0} \\
\hat{1} \\
\hat{1} \\
\hat{\sigma} \\
\end{array}$ & 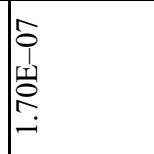 & $\begin{array}{l}\hat{0} \\
\hat{1} \\
m \\
m \\
m \\
-1\end{array}$ & 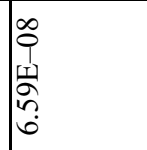 & 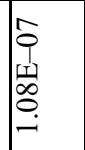 & $\begin{array}{l}\infty \\
0 \\
1 \\
1 \\
2 \\
\infty \\
\dot{m}\end{array}$ \\
\hline 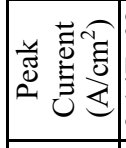 & 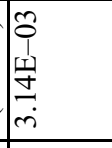 & $\begin{array}{l}m \\
0 \\
1 \\
1 \\
0 \\
n \\
i n \\
n\end{array}$ & 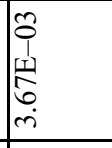 & $\begin{array}{c}0 \\
0 \\
1 \\
\tilde{1} \\
0 \\
0 \\
-1 \\
-1\end{array}$ & 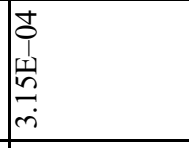 & 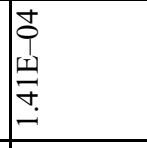 & 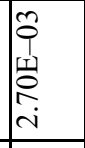 & 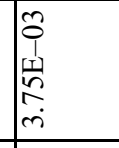 \\
\hline 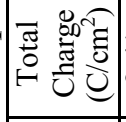 & $\underset{7}{\vec{F}}$ & ì & 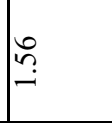 & $\hat{i}$ & & 命 & 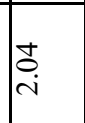 & a \\
\hline 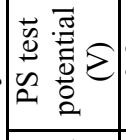 & ֻั & $\tilde{o}$ & $\stackrel{\sim}{o}$ & $\stackrel{n}{0}$ & $\stackrel{n}{o}$ & $\tilde{o}$ & $\stackrel{\sim}{o}$ & $\tilde{o}$ \\
\hline 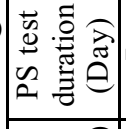 & r & I & r & $r$ & $r$ & r & N & r \\
\hline 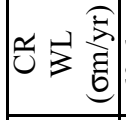 & 8 & in & ?n. & 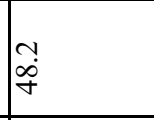 & $\stackrel{\circ}{\vec{\sim}}$ & $\mid \begin{array}{l}n \\
\infty \\
\infty\end{array}$ & $\hat{\sigma}$ & 茫 \\
\hline 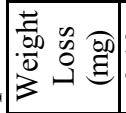 & $\frac{m}{a}$ & $\bar{\partial}_{\infty}$ & 声 & $\overrightarrow{\stackrel{N}{r}}$ & $\stackrel{\tilde{n}}{\tilde{r}}$ & $\stackrel{\infty}{\stackrel{\infty}{\longrightarrow}}$ & $\sqrt[n]{n}$ & 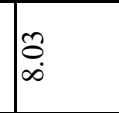 \\
\hline $\begin{array}{l}0 \\
\text { : } 0 \\
\ominus\end{array}$ & 8 & \& & 8 & 8 & 8 & \& & 8 & 8 \\
\hline $\begin{array}{l}0 \\
\stackrel{0}{\Xi} \\
0 \\
0 \\
0\end{array}$ & 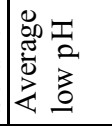 & 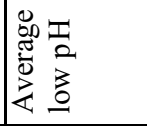 & 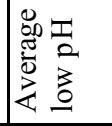 & 施苞 & 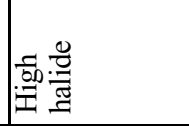 & 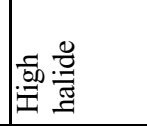 & 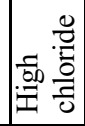 & 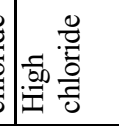 \\
\hline 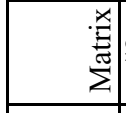 & 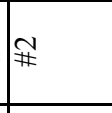 & 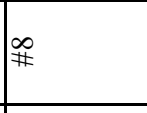 & $\mid \infty$ & 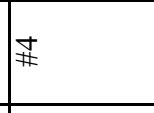 & 迅 & $\nRightarrow$ & 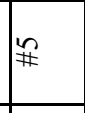 & $\nexists$ \\
\hline$\stackrel{\vec{v}}{\oplus} \theta$ & $\begin{array}{l}n \\
0 \\
0 \\
0 \\
0 \\
0 \\
0\end{array}$ & 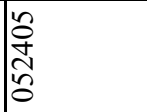 & 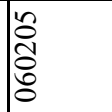 & $\begin{array}{l}2 \\
8 \\
0 \\
2\end{array}$ & $\begin{array}{l}n \\
8 \\
8 \\
0 \\
2\end{array}$ & $\begin{array}{l}n \\
2 \\
2 \\
2 \\
2\end{array}$ & 角 & 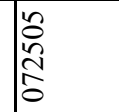 \\
\hline
\end{tabular}




\begin{tabular}{|c|c|c|c|c|c|c|c|c|c|}
\hline 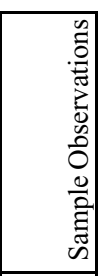 & 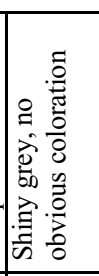 & 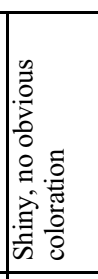 & 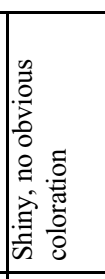 & 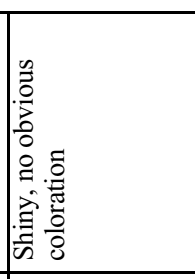 & & 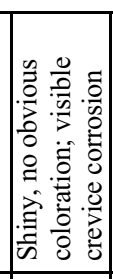 & 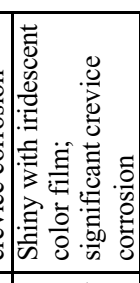 & 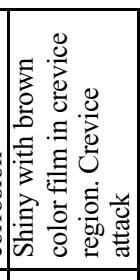 & 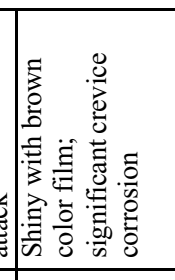 \\
\hline 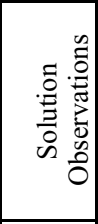 & 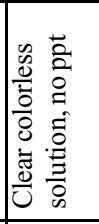 & 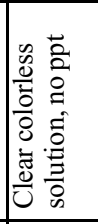 & 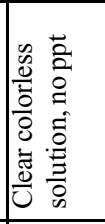 & 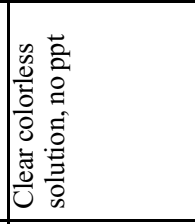 & & 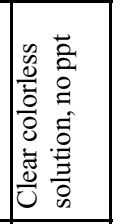 & 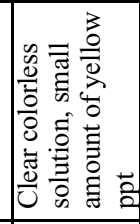 & 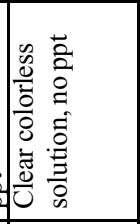 & 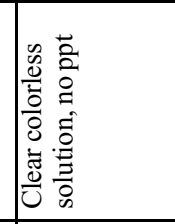 \\
\hline 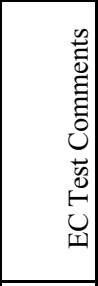 & 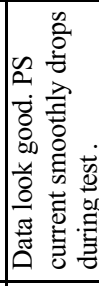 & 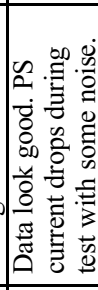 & 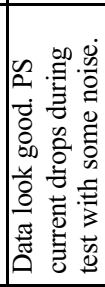 & 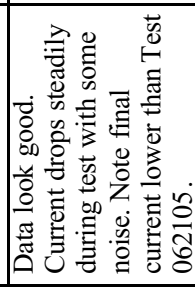 & 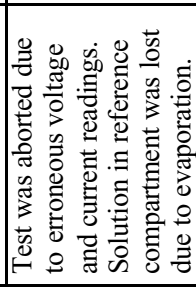 & 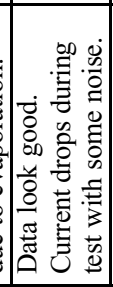 & 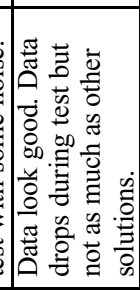 & 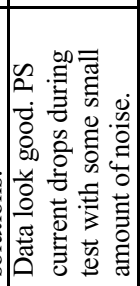 & 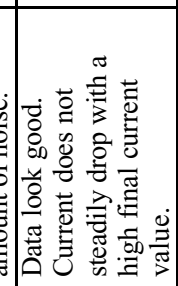 \\
\hline ช & 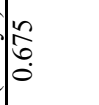 & $\tilde{\widehat{N}}$ & $\stackrel{0}{9}$ & 竞 & & $\hat{\vec{i}}$ & 穴 & $\stackrel{n}{\underline{z}}$ & m \\
\hline 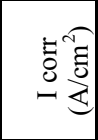 & $\mid \begin{array}{l}\infty \\
0 \\
1 \\
1 \\
0 \\
0 \\
0\end{array}$ & 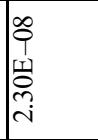 & 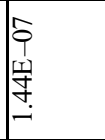 & $\begin{array}{l}\infty \\
0 \\
0 \\
\\
0 \\
0 \\
0\end{array}$ & & 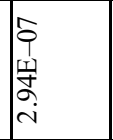 & 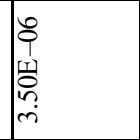 & 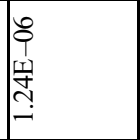 & 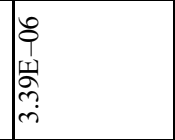 \\
\hline 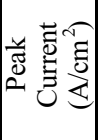 & 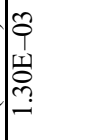 & 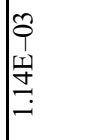 & 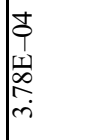 & $\begin{array}{l}\dot{y} \\
0 \\
\underline{1} \\
\tilde{\omega} \\
\hat{n} \\
\dot{n}\end{array}$ & & 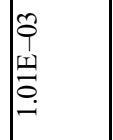 & 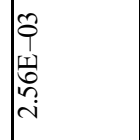 & $\begin{array}{l}\dot{+} \\
0 \\
\text { 山्ل } \\
\text { b } \\
\stackrel{i}{i}\end{array}$ & $\begin{array}{l}\dot{t} \\
0 \\
\omega \\
\infty \\
\infty \\
i\end{array}$ \\
\hline 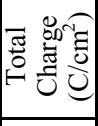 & $\begin{array}{l}\infty \\
\infty \\
i \\
\end{array}$ & $\mid \begin{array}{l}\tilde{\infty} \\
0 \\
0 \\
0\end{array}$ & o. & $\stackrel{n}{n}$ & & $\stackrel{n}{=}$ & 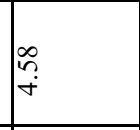 & ì & $\underset{\sim}{\tilde{n}}$ \\
\hline 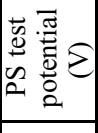 & $\stackrel{0}{0}$ & กั & ồ & Na & & $\stackrel{n}{o}$ & $\tilde{o}$ & $\tilde{o}$ & $\tilde{o}$ \\
\hline 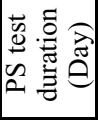 & | & r & 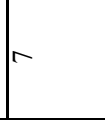 & 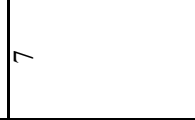 & & $\wedge$ & 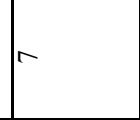 & r & 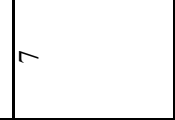 \\
\hline 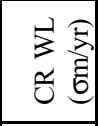 & 8 & ñ. & $\hat{\stackrel{\sim}{s}}$ & $\vec{m}$ & & $\frac{m}{m}$ & $\stackrel{\ominus}{\infty}$ & $\ddot{c}$ & 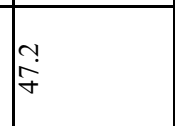 \\
\hline 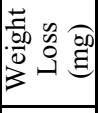 & 足 & $\stackrel{\sim}{\sim}$ & o. & 商 & 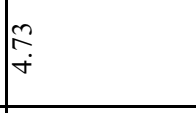 & $\vec{\infty}$ & $\underset{\pi}{\approx}$ & $\underset{\infty}{ \pm}$ & 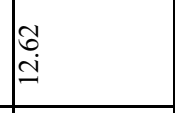 \\
\hline 急。 & 8 & $\stackrel{2}{2}$ & 8 & \& & 2 & 8 & \& & 8 & \& \\
\hline 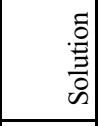 & 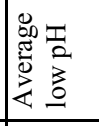 & 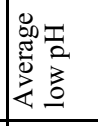 & 密密 & 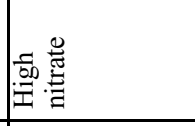 & 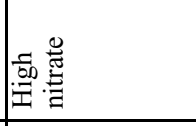 & 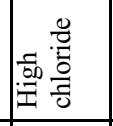 & 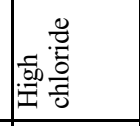 & 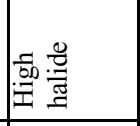 & 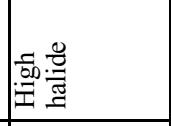 \\
\hline 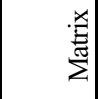 & & $\underset{\#}{\#}$ & $\frac{6}{\#}$ & $\underset{\#}{\#}$ & $\underset{\nexists}{\mathbb{N}}$ & 童 & $\cong$ & $\frac{\infty}{\#}$ & $\underset{\#}{\#}$ \\
\hline $\begin{array}{c}O \\
\stackrel{\overrightarrow{8}}{\leftrightarrow} \\
\stackrel{\leftrightarrow}{\oplus}\end{array}$ & & 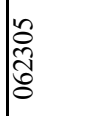 & 亮 & $\begin{array}{l}1 \\
0 \\
0 \\
0 \\
0 \\
0\end{array}$ & $\begin{array}{l}n \\
\frac{0}{2} \\
0 \\
0 \\
0\end{array}$ & $\frac{n}{\stackrel{2}{0}}$ & 总 & \begin{tabular}{l}
0 \\
0 \\
$\vdots$ \\
\hdashline \\
8
\end{tabular} & 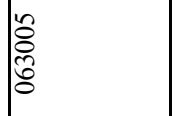 \\
\hline
\end{tabular}


The corrosion rates calc ulated from weight loss $(42.8 \pm 18.6 \mathrm{Pm} / \mathrm{yr}$ for cylinders specimens and $43.4 \pm 23.6 \mathrm{Pm} / \mathrm{yr}$ for crevice specimens) are greater than those calculated from $\mathrm{I}_{\text {corr }}(1.27 \pm 0.60 \mathrm{Pm} / \mathrm{yr}$ for cylinder specimens and $11.1 \pm 16.7 \mathrm{Pm} / \mathrm{yr}$ for crevice specimens). This can be explained by considering the difference in the way the two rates are determined. Figure 4 shows a hypothetical PS curve in the absence of crevice corrosion. The curve is split into two regions. Region I occurs immediately following polarization of the specimen and is associated with rapid dissolution of the reactive secondary phase where the current decreases as the secondary phase exposed to the surface is consumed. Region II is where the current drops at a much slower rate as the base material undergoes passivation. The corrosion rate due to weight loss considers all the processes during the test including removal of the secondary phase. The corrosion rate calculated from $\mathrm{I}_{\text {corr }}$ is determined from the final current measured in Region II where the secondary phase has been removed and passive currents of less than $100 \mathrm{nA} / \mathrm{cm}^{2}$ are observed. The corrosion rate calculated from $\mathrm{I}_{\text {corr }}$ is considered more indicative of the true long-term corrosion rate as the gadolinide will be selectively removed from the surface by pickling processes or by moist environments for long periods. For tests where crevice corrosion is observed, the values for the two corrosion rates are much closer due to the higher $\mathrm{I}_{\text {corr }}$ value. It is expected that the commercially available plate product that will be used for the waste package basket will have a hot rolled, annealed, and pickled finish The pickling process used to remove the oxide scale formed during the hot rolling and annealing of the plate is expected to remove the exposed gadolinide. This material would probably exhibit Region II behavior.

Other observations can be made from the tests results in Tables 7 and 8 . The maximum currents for all the tests are between $5.5 \mathrm{~mA} / \mathrm{cm}^{2}$ and $0.141 \mathrm{~mA} / \mathrm{cm}^{2}$ and always occur near the initiation of the tests. The total charge tracks the weight loss values, as the charge can be related to mass dissolved through Faradaic relationships. The solutions are all colorless with the exception of one crevice Test 080205. Blackish-brown films were observed in the four specimens that experienced significant crevice corrosion, isolated to the crevice region. Figure 5 shows a photograph of the damage caused in Test 063005 (after descaling) by crevice corrosion of the specimens.

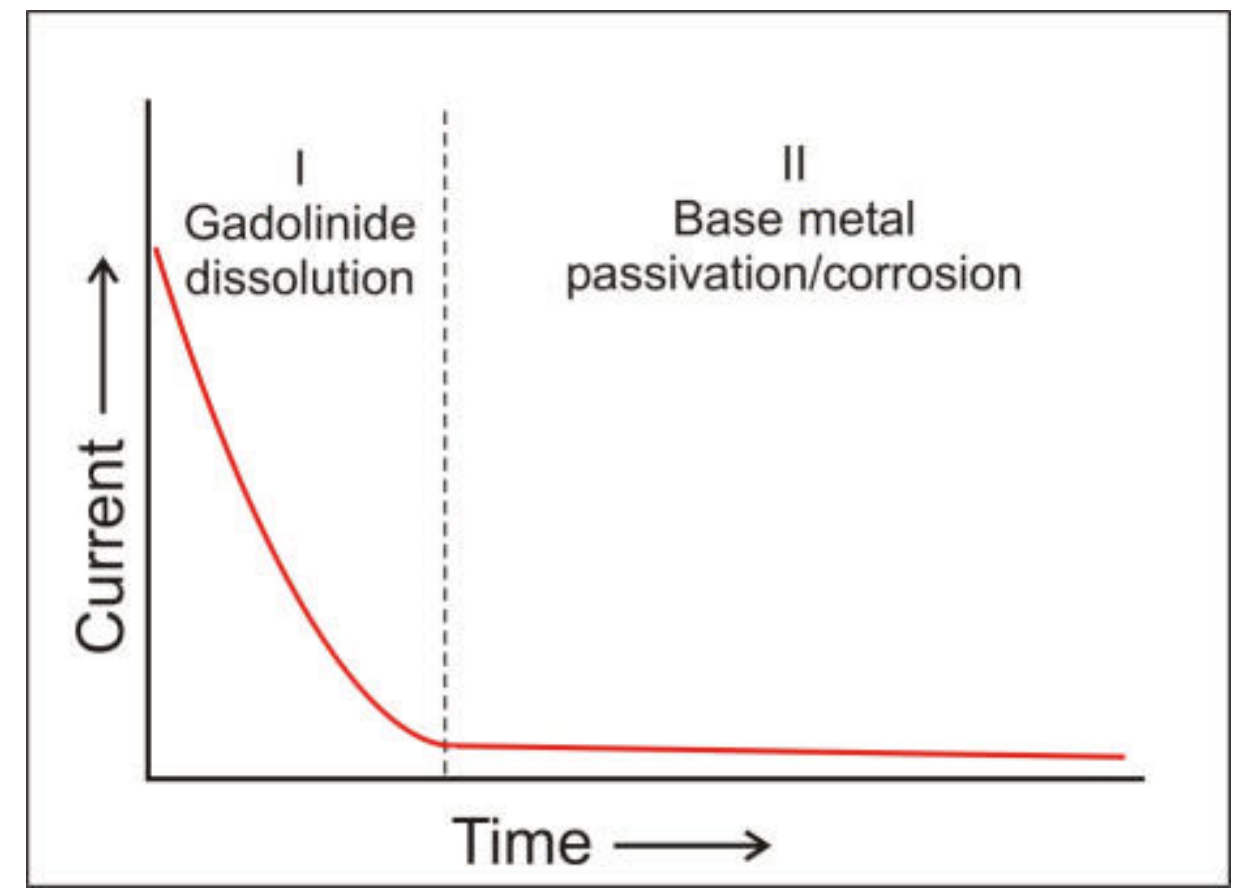

Figure 4. Diagram of a hypothetical potentiostatic curve for Ni-Cr-Mo-Gd alloy demonstrating two regions of behavior. 


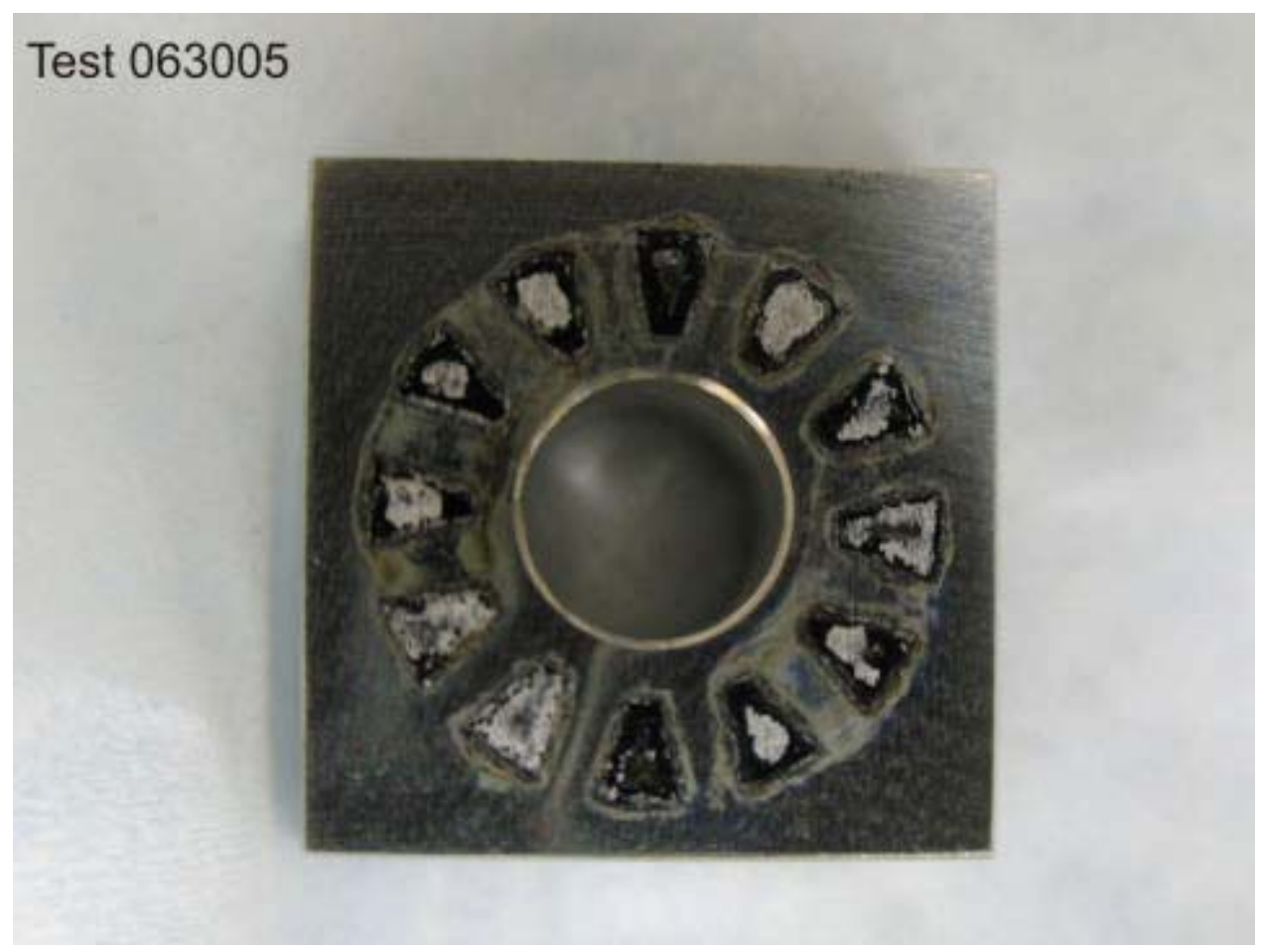

Figure 5. Photograph of the crevice specimen from Test 063005 showing extensive crevice attack.

\subsection{Morphology of Corrosion Specimens}

To determine the type of damage incurred by the corrosion tests, SEM was used to image the specimen surfaces after the tests. This was performed following specimen descaling, although some remaining precipitate was observed on some of the specimens. The corrosion-induced damage can be divided into two types: 1) secondary phase dissolution (gadolinide), where shallow pitting on the surface is observed and 2) primary phase etching (austenite) under creviced regions in the High halide and High chloride solutions.

Complete secondary phase dissolution was observed under most conditions. An exception to this is in the High halide solution where fluoride ions appeared to impede that process in PS tests due to the formation of insoluble $\mathrm{GdF}_{3}$, which will be discussed in the solution analysis section. ${ }^{17}$ Figure $6 \mathrm{~A}$ shows a backscatter SEM image of the boldly exposed surface of the crevice specimen used in Test 063005, a PS test in High halide solution at $90^{\circ} \mathrm{C}$. In this image, the bright phase is due to the high $\mathrm{Z} \mathrm{Gd}$ atoms of the secondary phase. These particles are intact although significant micro pitting is observed. In other solutions, the secondary phase appears readily soluble, as evidenced by the clear removal of a secondary phase particle in Figure 6B, from the specimen used in Test 072505 , at $90^{\circ} \mathrm{C}$ in High chloride solution. A high magnification of the structure of the pit bottom left by the removed secondary phase is shown in Figure 6C, from Test 042605, a PD test in Average low pH solution. This image demonstrates the shallow nature of this pitting damage.

Significant damage to the primary austenite phase was observed in tests employing crevice specimens with greater halide to nitrate ratios (High halide and High chloride solutions). This damage was most severe in potentiostatic tests due to the time the specimen spent ( 7 days) at the aggressive condition ( $0.2 \mathrm{~V}$ vs. ref). In all the PS tests using crevice specimens in these solutions, extensive damage could be observed by the naked eye. A low magnification SEM image shown in Figure 7A shows damage 
from Test 063005 , a PS test in High halide solution at $90^{\circ} \mathrm{C}$. The damage essentially corresponds to the specimen area in contact with the ceramic crevice forming tooth. This contact area is outlined by precipitate that was not removed by the descaling procedure and shows greater damage in the area next to this precipitate. A higher magnification of this interface region is shown in Figure 7B, taken from the outer edge of the crevice tooth shown in Figure 7A. This image contrasts the damage from under the crevice former (right) to the relatively undamaged region on the upper left that was outside the crevice former. A line of precipitate separates these two regions, where metals (corrosion products) dissolved in the low $\mathrm{pH}$ environment under the crevice precipitate at the crevice boundary where the $\mathrm{pH}$ is moderated. High magnification of the damaged region is shown in Figure 7C. The damage under the crevice former appears to be an etching of the base material and no longer resembles the morphology due to removal of the secondary phase. Similar attack has been observed for Alloy 22 under crevice formers under more aggressive conditions after PS tests. ${ }^{18}$ Evidence of crevice attack of the base material has been observed in PD tests in High chloride solution (Test 072605). The location and morphology of the damage appears similar to what was observed above but not as extensive.
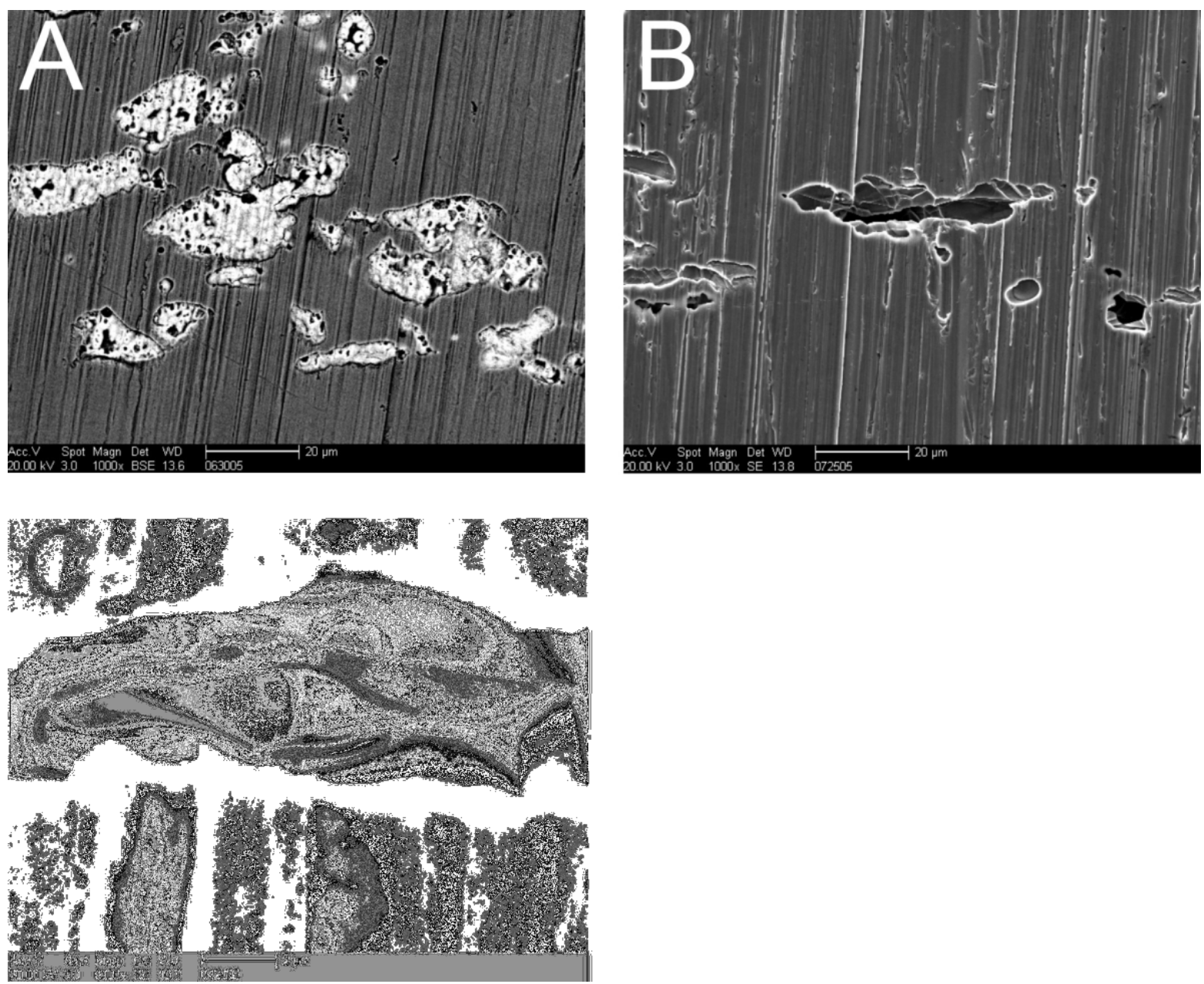

Figure 6. SEM micrographs of test specimens: (a) Test 063005 specimen from boldly exposed region, (b) Test 072505 specimen, and (c) high magnification of pot from Test 042605 specimen. 

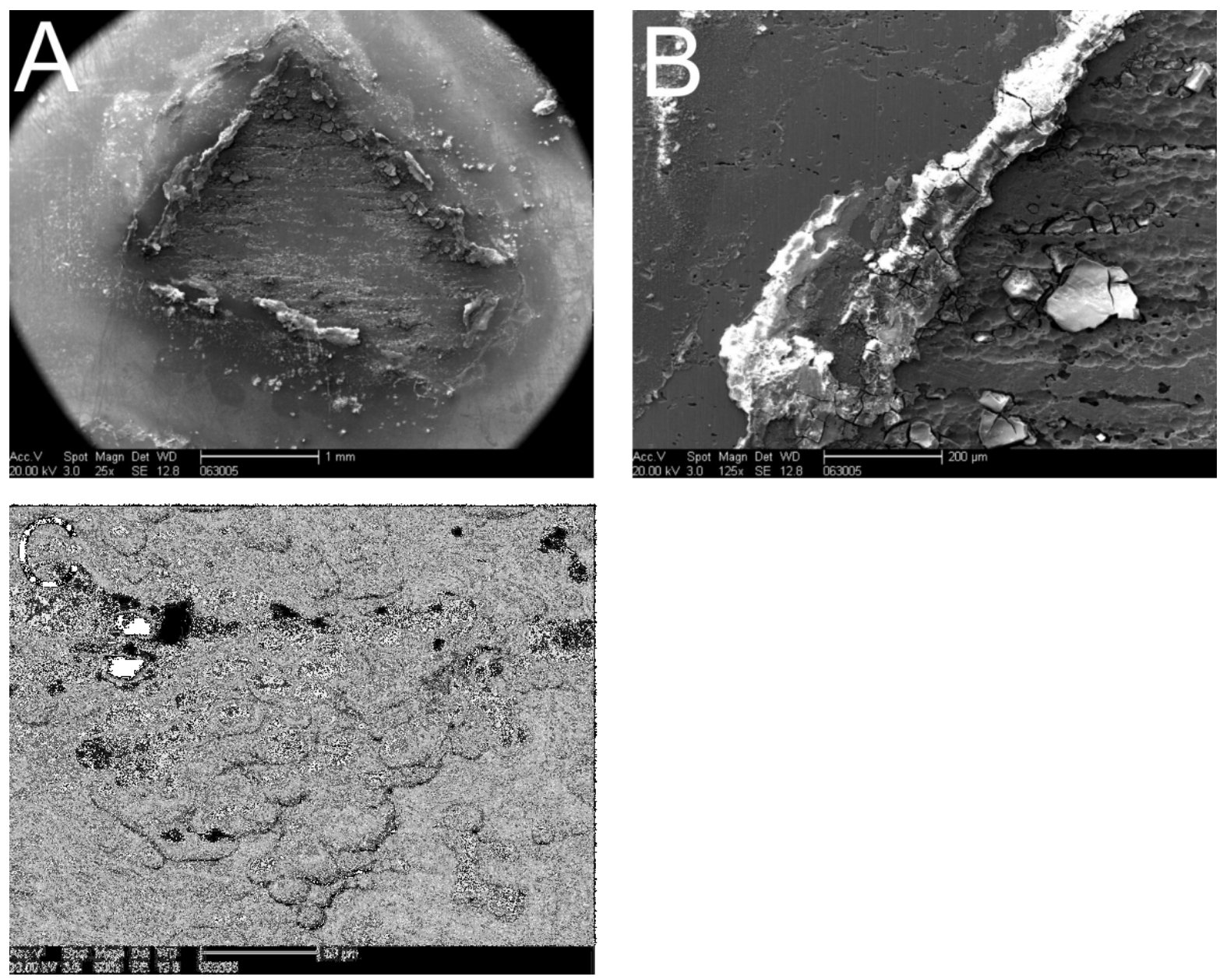

Figure 7. SEM micrographs of crevice damage on Test 063005 specimen at various magnifications.

\subsection{Analysis of Corrosion Test Solutions}

The corrosion test solutions were analyzed after performing the tests to determine the amount of metal dissolved into solution. An aliquot of solution was collected following the test and sent to a qualified laboratory for metal analysis for the primary constituents ( $\mathrm{Ni}, \mathrm{Cr}, \mathrm{Mo}$, and $\mathrm{Gd}$ ) by inductively coupled plasma atomic emission spectroscopy (ICP-AES) and solution $\mathrm{pH}$. The results are shown in Table 9. From each of the constituent metal concentrations the total metal concentration was calculated. This can be compared directly with the values from weight loss measurements (also provided for comparison). Because the testing solution was one liter, the values can be compared directly and should result in agreement if precipitation does not influence the solubility of the metal. For tests where solution precipitation was not observed, the total metal concentration and weight loss values agree fairly well. This provides confidence in both of the methods of analysis. The $\mathrm{pH}$ values are of less significance as reactions at the electrodes and dissolved components will shift the unbuffered solution. As an example, Test 070105 had the lowest $\mathrm{pH}$ value and was a test that had a reference electrode failure (capillary leaked) and excessive current occurred. 
Table 9. Post-test solution analysis results.

\begin{tabular}{|c|c|c|c|c|c|c|c|c|c|c|}
\hline $\begin{array}{l}\text { Test } \\
\text { ID }\end{array}$ & Matrix & Solution & $\begin{array}{c}\text { Temp } \\
\left({ }^{\circ} \mathrm{C}\right) \\
\end{array}$ & $\begin{array}{c}\text { Solution } \\
\text { Analysis Ni } \\
\mu \mathrm{g} / \mathrm{L} \\
\end{array}$ & $\begin{array}{c}\text { Solution } \\
\text { Analysis } \mathrm{Cr} \\
\mu \mathrm{g} / \mathrm{L} \\
\end{array}$ & $\begin{array}{c}\text { Solution } \\
\text { Analysis Mo } \\
\mu \mathrm{g} / \mathrm{L} \\
\end{array}$ & $\begin{array}{c}\text { Solution } \\
\text { Analysis Gd } \\
\mu \mathrm{g} / \mathrm{L}\end{array}$ & $\begin{array}{c}\text { Total Solution } \\
\text { Analysis wt } \\
\mathrm{mg} / \mathrm{L} \\
\end{array}$ & $\begin{array}{l}\text { Wt } \\
\text { Loss } \\
(\mathrm{mg}) \\
\end{array}$ & $\mathrm{pH}$ \\
\hline \multicolumn{11}{|c|}{ PD Cylinder specimens } \\
\hline 040405 & $\# 26$ & $\begin{array}{l}\text { Average low } \\
\mathrm{pH}\end{array}$ & 60 & 41200 & 5100 & 5620 & 10200 & 62.12 & 28.53 & 7.05 \\
\hline 040605 & \#26 & $\begin{array}{l}\text { Average low } \\
\mathrm{pH}\end{array}$ & 60 & 6330 & 645 & 763 & 1460 & 9.20 & 14.99 & 6.96 \\
\hline 040805 & \#26 & $\begin{array}{l}\text { Average low } \\
\mathrm{pH}\end{array}$ & 60 & 22100 & 1960 & 2420 & 5000 & 31.48 & 13.13 & 7.09 \\
\hline 041205 & \#26 & $\begin{array}{l}\text { Average low } \\
\mathrm{pH}\end{array}$ & 60 & 69700 & 8420 & 8710 & 11500 & 98.33 & 24.74 & 7.26 \\
\hline 042205 & \#26 & $\begin{array}{l}\text { Average low } \\
\mathrm{pH}\end{array}$ & 60 & 14800 & 1840 & 2160 & 2790 & 21.59 & 18.60 & 7.04 \\
\hline 042505 & \#32 & $\begin{array}{l}\text { Average low } \\
\mathrm{pH}\end{array}$ & 90 & 140000 & 18800 & 23200 & 12500 & 194.50 & 66.36 & 7.20 \\
\hline 042605 & $\# 32$ & $\begin{array}{l}\text { Average low } \\
\mathrm{pH}\end{array}$ & 90 & 5190 & 116 & 142 & 1820 & 7.27 & 7.04 & 5.06 \\
\hline 042705 & $\# 30$ & High halide & 60 & 5450 & 757 & 751 & 14.1 & 6.97 & 6.82 & 4.76 \\
\hline 042805 & $\# 30$ & High halide & 60 & 1950 & 276 & 276 & 1.31 & 2.50 & 7.52 & 4.81 \\
\hline 050205 & $\# 36$ & High halide & 90 & 22800 & 3570 & 3810 & 176 & 30.36 & 24.79 & 5.76 \\
\hline 050405 & \#36 & High halide & 90 & 35000 & 4160 & 4790 & 640 & 44.59 & 25.12 & 6.78 \\
\hline 050505 & $\# 28$ & High nitrate & 60 & 3140 & 212 & 242 & 414 & 4.01 & 7.03 & 6.51 \\
\hline 050605 & \#28 & High nitrate & 60 & 7540 & 323 & 343 & 2400 & 10.61 & 13.55 & 6.72 \\
\hline 051705 & $\# 34$ & High nitrate & 90 & 6590 & 360 & 209 & 2220 & 9.38 & 9.03 & 3.54 \\
\hline 051805 & $\# 34$ & High nitrate & 90 & 9580 & 946 & 1500 & 2250 & 14.28 & 10.97 & 6.64 \\
\hline 071205 & $\# 29$ & High chloride & 60 & 7190 & 61.8 & 98.8 & 1490 & 8.84 & 10.98 & 6.29 \\
\hline 071805 & $\# 35$ & High chloride & 90 & 5980 & 293 & 238 & 150 & 6.66 & 12.28 & 6.70 \\
\hline 072005 & \#35 & High chloride & 90 & 6070 & 411 & 363 & 160 & 7.00 & 10.40 & 6.49 \\
\hline \multicolumn{11}{|c|}{ PD Crevice specimens } \\
\hline 051905 & \#44 & $\begin{array}{l}\text { Average low } \\
\mathrm{pH}\end{array}$ & 90 & 8990 & 258 & 268 & 1780 & 11.30 & 12.16 & 6.34 \\
\hline 052005 & $\# 44$ & $\begin{array}{l}\text { Average low } \\
\mathrm{pH}\end{array}$ & 90 & 10300 & 417 & 324 & 2240 & 13.28 & 13.26 & 6.06 \\
\hline 060105 & \#38 & $\begin{array}{l}\text { Average low } \\
\mathrm{pH}\end{array}$ & 60 & 5530 & 73.8 & 152 & 1550 & 7.31 & 8.18 & 6.22 \\
\hline 060305 & \#38 & $\begin{array}{l}\text { Average low } \\
\mathrm{pH}\end{array}$ & 60 & 11100 & 857 & 978 & 3140 & 16.08 & 18.70 & 6.91 \\
\hline 060605 & $\# 42$ & High halide & 60 & 14100 & 1850 & 2090 & 26.3 & 18.07 & 21.02 & 5.25 \\
\hline 060705 & $\# 42$ & High halide & 60 & 9720 & 1040 & 1230 & 16.5 & 12.01 & 12.94 & 4.87 \\
\hline 060805 & $\# 40$ & High nitrate & 60 & 14100 & 345 & 335 & 5040 & 19.82 & 20.54 & 5.49 \\
\hline 071405 & $\# 41$ & High chloride & 60 & 10600 & 308 & 267 & 4380 & 15.56 & 20.69 & 6.83 \\
\hline 071905 & $\# 47$ & High chloride & 90 & 4230 & 230 & 277 & 1050 & 5.79 & 14.74 & 6.70 \\
\hline 072105 & $\# 41$ & High chloride & 60 & 1910 & 90.4 & 135 & 292 & 2.43 & 7.50 & 6.24 \\
\hline 072605 & $\# 47$ & High chloride & 90 & 3410 & 65.8 & 69.7 & 237 & 3.78 & 17.83 & 6.47 \\
\hline 072705 & $\# 46$ & High nitrate & 90 & 2420 & 112 & 134 & 78.5 & 2.74 & 9.21 & 6.28 \\
\hline 072905 & $\# 46$ & High nitrate & 90 & 7060 & 119 & 58.4 & 1670 & 8.91 & 10.48 & 5.93 \\
\hline 080905 & $\# 48$ & High halide & 90 & 15900 & 1980 & 2300 & 846 & 21.03 & 28.00 & 6.95 \\
\hline 081005 & $\# 48$ & High halide & 90 & 10900 & 1090 & 1400 & 191 & 13.58 & 17.34 & 6.34 \\
\hline
\end{tabular}


Table 9. (continued).

\begin{tabular}{|c|c|c|c|c|c|c|c|c|c|c|}
\hline $\begin{array}{c}\text { Test } \\
\text { ID } \\
\end{array}$ & Matrix & Solution & $\begin{array}{c}\text { Temp } \\
\left({ }^{\circ} \mathrm{C}\right) \\
\end{array}$ & $\begin{array}{c}\text { Solution } \\
\text { Analysis Ni } \\
\mu \mathrm{g} / \mathrm{L} \\
\end{array}$ & $\begin{array}{c}\text { Solution } \\
\text { Analysis Cr } \\
\mu \mathrm{g} / \mathrm{L} \\
\end{array}$ & \begin{tabular}{|c|} 
Solution \\
Analysis Mo \\
$\mu \mathrm{g} / \mathrm{L}$ \\
\end{tabular} & $\begin{array}{c}\text { Solution } \\
\text { Analysis Gd } \\
\mu \mathrm{g} / \mathrm{L} \\
\end{array}$ & $\begin{array}{c}\text { Total Solution } \\
\text { Analysis wt } \\
\mathrm{mg} / \mathrm{L} \\
\end{array}$ & $\begin{array}{l}\text { Wt } \\
\text { Loss } \\
(\mathrm{mg}) \\
\end{array}$ & $\mathrm{pH}$ \\
\hline 081105 & $\# 40$ & High nitrate & 60 & 6290 & 193 & 203 & 2070 & 8.76 & 12.07 & 6.68 \\
\hline \multicolumn{11}{|c|}{ PS Cylindrical specimens } \\
\hline 050305 & $\# 2$ & $\begin{array}{l}\text { Average low } \\
\mathrm{pH}\end{array}$ & 60 & 6710 & 112 & 3.75 & 3340 & 10.17 & 9.13 & 3.81 \\
\hline 050905 & \#6 & High halide & 60 & 3330 & 51.2 & 8.22 & 5.86 & 3.40 & 3.23 & 5.76 \\
\hline 051605 & $\# 4$ & High nitrate & 60 & 4920 & 1.1 & 14.4 & 2120 & 7.06 & 7.21 & 6.10 \\
\hline 052405 & $\# 8$ & $\begin{array}{l}\text { Average low } \\
\mathrm{pH}\end{array}$ & 90 & 6590 & 14.2 & 18.7 & 3290 & 9.91 & 8.91 & 4.30 \\
\hline 052505 & $\# 12$ & High halide & 90 & 1370 & 51.3 & 27.9 & 4.18 & 1.45 & 1.28 & 4.98 \\
\hline 060205 & $\# 8$ & $\begin{array}{l}\text { Average low } \\
\mathrm{pH}\end{array}$ & 90 & 5430 & 61.1 & 7.66 & 2660 & 8.16 & 6.04 & 3.78 \\
\hline 071505 & $\# 5$ & High chloride & 60 & 5480 & 79.9 & 13.5 & 2810 & 8.38 & 7.51 & 3.89 \\
\hline 072505 & $\# 11$ & High chloride & 90 & 4900 & 0.917 & 40.7 & 1850 & 6.79 & 8.03 & 6.24 \\
\hline \multicolumn{11}{|c|}{ PS Crevice specimens } \\
\hline 061305 & $\# 14$ & $\begin{array}{l}\text { Average low } \\
\mathrm{pH}\end{array}$ & 60 & 15000 & ND & 22.5 & 6770 & 21.79 & 17.50 & 5.91 \\
\hline 061405 & $\# 18$ & High halide & 60 & 9740 & 345 & 229 & 3.42 & 10.32 & 8.14 & 5.00 \\
\hline 062105 & $\# 16$ & High nitrate & 60 & 5350 & ND & 23.3 & 2060 & 7.43 & 7.09 & 6.12 \\
\hline 062305 & \#20 & $\begin{array}{l}\text { Average low } \\
\mathrm{pH}\end{array}$ & 90 & 1940 & 0.637 & 44.5 & 34 & 2.02 & 4.32 & 6.37 \\
\hline 063005 & \#24 & High halide & 90 & 7080 & 6.92 & 163 & 0.873 & 7.25 & 12.62 & 6.47 \\
\hline 070105 & \#22 & High nitrate & 90 & 35700 & 5550 & 2830 & 756 & 44.84 & 4.73 & 2.80 \\
\hline 070605 & \#22 & High nitrate & 90 & 7120 & ND & 60.6 & 3010 & 10.19 & 10.39 & 5.51 \\
\hline 080105 & $\# 17$ & High chloride & 60 & 5600 & 0.971 & 16.8 & 1220 & 6.84 & 8.31 & 6.37 \\
\hline 080205 & \#23 & High chloride & 90 & 12100 & 97.1 & 23.8 & 148 & 12.37 & 23.17 & 6.48 \\
\hline
\end{tabular}

Gadolinium is observed in all the conditions. The secondary phase is known to dissolve in these tests as observed by SEM analysis following the tests. In the High halide solution, gadolinium complexes with fluoride ions in solution forming the insoluble compound $\mathrm{GdF}_{3}$, (see Reference 17) leading to very low concentrations of gadolinium in the post-test solutions. This sequestration by fluoride ions to form $\mathrm{GdF}_{3}$ can allow this compound to be homogeneously distributed throughout the breached waste package by replating on metallic surfaces or moving to the sludge in the bottom of the waste package. The $\mathrm{GdF}_{3}$ could possibly then form a new, gadolinium rich surface layer on the fuel basket plates.

Nickel, the primary constituent of the primary and secondary phases, was the most abundant metal in solution for all tests. Nickel would thus be involved in all dissolution processes.

Chromium and molybdenum are located in the primary austenite phase as the secondary phase does not have a significant concentration of chromium and molybdenum (see Reference 8). The PD tests show more dissolution of chromium and molybdenum, as these elements are dissolved in the transpassive region at the anodic limit. The values for chromium and molybdenum are lower for the PS tests, as potentials where transpassive dissolution occurs were not accessed. One exception is Test 070105, a test that was flawed due to a reference failure (potential not controlled). The PD tests that used $1.0 \mathrm{~V}$ as the reversal potential (versus $0.8 \mathrm{~V}$ for most tests) showed the most base metal constituents (Ni, Mo, and $\mathrm{Gd}$ ) due to deeper penetration into the transpassive region. 


\subsection{Microstructural Analysis}

A typical microstructure for the Ni-Cr-Mo-Gd alloy is shown in Figure 8. This image is from a cross-sectioned cylindrical specimen from Test 072505, which is a PS test in the High chloride solution at $90^{\circ} \mathrm{C}$. The microstructure consists of a Ni-Cr-Mo austenitic matrix with a composition similar to UNS N06455 (darker structure) and a dispersed secondary phase (lighter structure) with a crystal structure based on the $\mathrm{Ni}_{5} \mathrm{Gd}$ gadolinide. This secondary phase is generally found at the austenite grain boundaries. The $\mathrm{Ni}_{5} \mathrm{Gd}$ phase also contains small amounts of dissolved chromium and molybdenum (on the order of $1 \mathrm{wt} \%$ ) (see Reference 8 ). The size, shape, and distribution of the secondary phase evolves from its initial solidification morphology in the interdendritic area of the as cast ingot through hot rolling and heat treatment to the wrought structure illustrated here. Gadolinium has extremely limited solubility in the nickelaustenite matrix and has not been detected there. In general, the alloy can be described as one containing a hard dispersed secondary phase within a softer austenitic matrix.

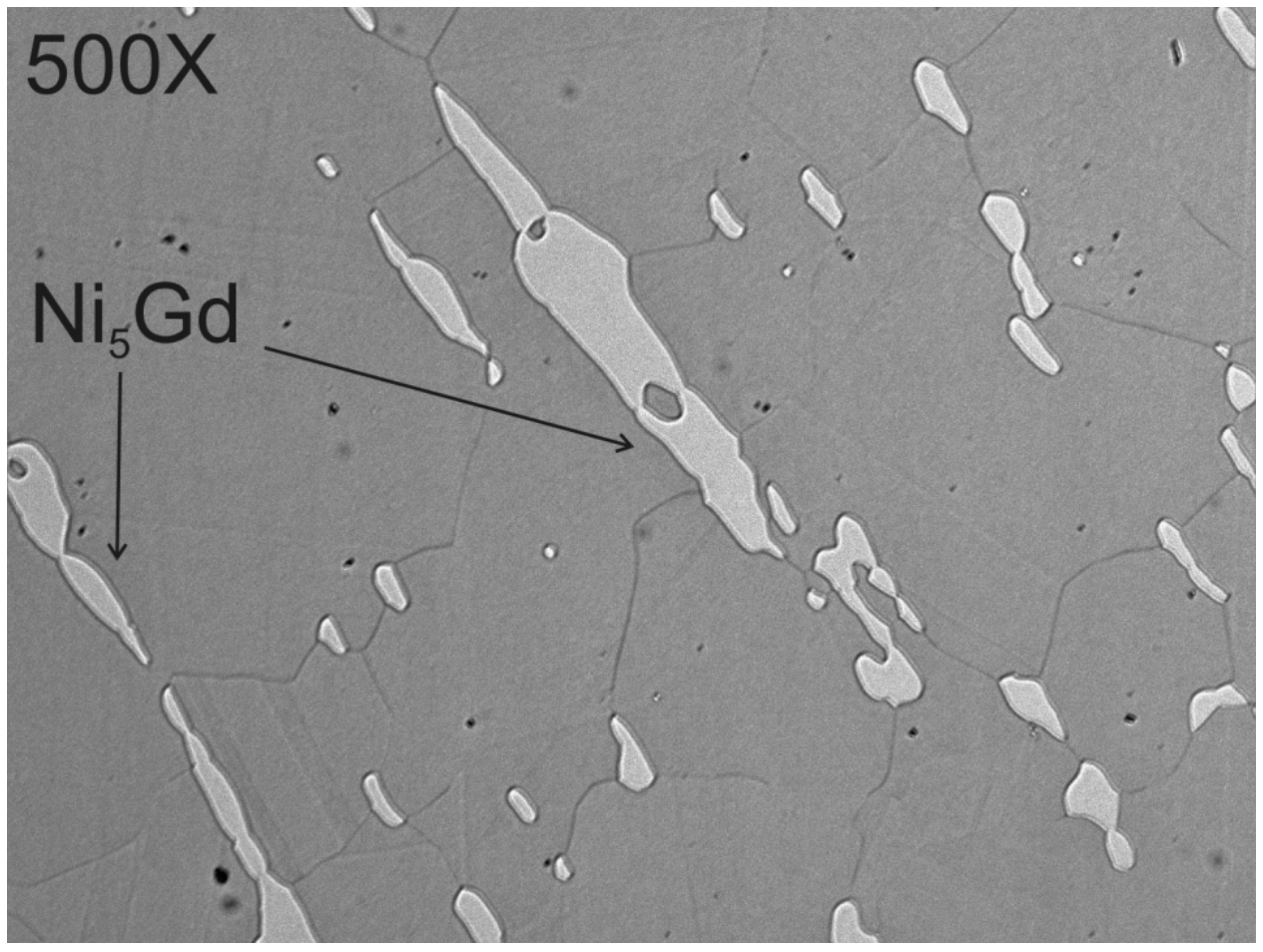

Figure 8. Light optical micrograph of cross-sectioned specimen at 500X magnification. 


\section{DISCUSSION AND CONCLUSIONS}

\subsection{Discussion of Results}

An important issue has arisen in the work presented in this report concerning the crevice corrosion of the alloy. Previous work with this alloy has examined boldly exposed specimens, which under a variety of conditions exhibited only secondary phase corrosion (see References 4 through 8 ). The damage observed in the PS tests in both solutions having higher concentrations of halides needs to be addressed. The crevices used here are formed using a very high torque (70 in.-lb). The combination of the MCA crevice formers, Teflon tape, and high torque values result in a crevice much tighter than anticipated in service. The potential chosen for the PS tests $(+0.2 \mathrm{~V})$ needs to be evaluated by long-term $\mathrm{E}_{\text {corr }}$ measurements to determine if the potential used in this testing is more positive than should be used and overly conservative. During long-term $\mathrm{E}_{\text {corr }}$ measurements for Alloy 22, only solutions with significant nitrate concentrations exhibited extremely positive $\mathrm{E}_{\text {corr }}$ values (above $0.1 \mathrm{~V}$ versus saturated silver chloride $[\mathrm{SSC}]$ ), a solution that contains a large concentration of nitrate ion (see Reference 19, Appendix V).

Intermediate $\mathrm{E}_{\text {corr }}$ values ( $0.031 \mathrm{~V}$ to $0.108 \mathrm{~V}$ versus SSC) were observed for Alloy 22 in simulated dilute water solution, which is the closest to the range of chemistries used in this work. Values in chloride solutions exhibited much lower $\mathrm{E}_{\text {corr }}$ values.

The enoblement (positive $\mathrm{E}_{\text {corr }}$ values) of Ni-Cr-Mo alloys was studied by Lloyd et al in $1 \mathrm{M} \mathrm{NaCl}$ plus $0.1 \mathrm{M} \mathrm{H}_{2} \mathrm{SO}_{4}$ solution. ${ }^{20}$ Ennoblement was observed for the higher chromium alloys (Alloy 22 and $\mathrm{C} 2000)$, but not for lower chromium alloys $(\mathrm{C}-4$ and $\mathrm{C} 276)$ at $75^{\circ} \mathrm{C}$. The alloys with higher chromium have $\mathrm{E}_{\text {corr }}$ values between $0.1 \mathrm{~V}$ to $0.25 \mathrm{~V}$ versus $\mathrm{Ag} / \mathrm{AgCl}(0.1 \mathrm{M} \mathrm{KCl})$, while the lower chromium alloys had $\mathrm{E}_{\text {corr }}$ values below $-0.2 \mathrm{~V}$ versus $\mathrm{Ag} / \mathrm{AgCl}(0.1 \mathrm{M} \mathrm{KCl})$. This is due to the extreme passive state of the alloys via a stable $\mathrm{Cr}_{2} \mathrm{O}_{3}$ inner layer film. The more negative values for $\mathrm{E}_{\text {corr }}$ were the result of a less stable inner layer film. The Ni-Cr-Mo-Gd alloy has a base material composition similar to C-4. Thus for the case where the Ni-Cr-Mo-Gd alloys are passive, the PS value of $0.2 \mathrm{~V}$ is likely close but slightly positive of the expected $\mathrm{E}_{\text {corr }}$ value. However, under conditions where localized (crevice) corrosion of the base material is observed, the $\mathrm{E}_{\text {corr }}$ is expected to shift significantly more negative as was observed for the lower chromium alloys (see Reference 20).

Another approach would be to consider the use of a higher chromium version of the alloy. One higher chromium version $(21.01 \%$ chromium $)$ of the Ni-Cr-Mo-Gd alloy demonstrated the increased corrosion resistance compared to the nominal ASTM B932-04 specification Ni-Cr-Mo-Gd alloy. ${ }^{21}$ Although crevice corrosion performance was not evaluated, it is anticipated that better performance would be observed.

PS testing of crevice corrosion has been performed on Alloy 22 in mixed chloride-nitrate solutions at near neutral $\mathrm{pH}$ values at $100^{\circ} \mathrm{C}$ (see References 18 and 22). These tests were performed in solutions with nitrate to chloride ratios similar to the High chloride solution used here, but approximately three orders of magnitude more concentrated. Extensive crevice corrosion was observed for Alloy 22 at $0.1 \mathrm{~V}$ versus SSC. The SSC is $0.043 \mathrm{~V}$ negative of the SCE-type reference electrode used in this work or adjusted is $0.143 \mathrm{~V}$ versus SCE. This is very similar to the value used in these tests. The crevice attack was eventually stifled due to the enlarging of tight crevices by metal dissolution. Such a mechanism could occur in this material although that may require longer tests to observe. Crevice corrosion occurs here under much more benign conditions likely due to the much lower corrosion resistance of this material (lower chromium level). The dissolution of secondary phase particles may also influence the crevice 
attack by providing a lower $\mathrm{pH}$ environment in the crevice region via metal ion dissolution. On boldly exposed areas, this should be less of a concern.

The measurement of repassivation potentials in the second sweep shows the potential for localized corrosion. The repassivation values tend to be between $0 \mathrm{~V}$ and $-0.1 \mathrm{~V}$ versus SCE under most conditions. Because the measured $\mathrm{E}_{\text {corr }}$ values are about $0.2 \mathrm{~V}$ negative of these values, more work is needed to further evaluate the potential for localized corrosion. Establishing the long-term value for $\mathrm{E}_{\text {corr }}$ is needed. In addition, the long-term $\mathrm{E}_{\text {corr }}$ for the alloy with the secondary phase dissolved should also be measured.

Under many conditions, the alloy performance in PS tests appears very promising. While the secondary phase is very reactive, the base material appears to be stable for boldly exposed specimens and in crevice specimens with higher $\mathrm{NO}_{3} / \mathrm{Cl}$ ratios. The corrosion rates measured in PS tests following the removal of surface exposed secondary phase are around $1 \mathrm{Pm} / \mathrm{yr}$ and in some cases somewhat lower. This rate is similar to rates determined in previous work performed under different conditions (see Reference 7). Figure 6 shows a cross-sectional diagram of the near surface region of the Ni-Cr-Mo-Gd alloy showing the processes that are thought to occur in these tests. First, selective removal of surface-exposed secondary phase occurs as evidenced by the morphology of the surface. This is followed by oxide growth processes and passivation of the surface exposed by the removal of the secondary phase. The corrosion rates measured here were after 7 days, where the current slowly decays after the initial steep decline (Figures 3 and 4). From previous exposure testing results from Alloy 22 (see Reference 19, Figure 7-1), it is expected that the corrosion rates would continue to decrease over time. This could be confirmed using longer term PS tests or by exposure testing materials in a manner similar to that performed for Alloy 22 in long-term corrosion tests mentioned above.

The LPR data collected prior to the PD tests show relatively large corrosion rates compared to those obtained from PS tests (both weight loss and $\mathrm{I}_{\text {corr }}$ derived). The values do not appear to correlate to the corrosive nature of the test as they did in PS tests. The LPR rates should be controlled by the dissolution rates of the secondary phase. These rates are expected to be quite high. Because the oxide film covering these secondary phase particles should not contain significant amounts of chromium based on previous examination of composition (see Reference 8), this phase is not very resistant to corrosion compared to the base material. It is possible that the corrosion rate of the secondary phase has a different corrosion rate dependence on environment than the base material. The corrosion rates obtained by LPR for Alloy 22 are about two orders of magnitude higher than the rates (slightly different environments) obtained by exposure testing for Alloy 22 (see Reference 19, Figures 6-11, 6-23, and 6-24). One explanation for the discrepancies between the two measurement methods might be the higher rates for a material immediately following immersion. The corrosion rates are known to drop with immersion time in long-term corrosion tests (see Reference 19, Figure 7-1), as was mentioned earlier. Also, LPR is used to define general corrosion rates, and the dissolution of the secondary phase is a localized phenomenon. For these reasons, it is thought that the LPR-measured values presented here are not as representative as those obtained in PS tests. In future testing, the LPR rate should be collected after the electrochemical test to determine the rate for the base material for comparison with the initially measured LPR value.

The general corrosion rate for Alloy C-4 has been measured in long-term corrosion tests in concentrated solutions considered for the waste package outer barrier. ${ }^{23}$ The most complete data set exists for the SAW solution (simulated acidified water, a concentrated acidified water solution), where the immersed coupons showed a decrease in corrosion rate from 6 months to 1 year of $0.08 \mathrm{Pm} / \mathrm{yr}$ to $0.04 \mathrm{Pm} / \mathrm{yr}$ at $60^{\circ} \mathrm{C}$ and $0.13 \mathrm{Pm} / \mathrm{yr}$ to $0.05 \mathrm{Pm} / \mathrm{yr}$ at $90^{\circ} \mathrm{C}$. This shows a decrease in corrosion rate for Alloy $\mathrm{C}-4$ with exposure time and also provides a value for comparison with the secondary phase depleted rates obtained by $\mathrm{I}_{\text {corr }}$ measurements of the Ni-Cr-Mo-Gd alloy tested here. The values for the Ni-Cr-Mo-Gd alloy, albeit in more dilute solutions, are near $1 \mathrm{Pm} / \mathrm{yr}$ (in noncrevice corrosion tests) 


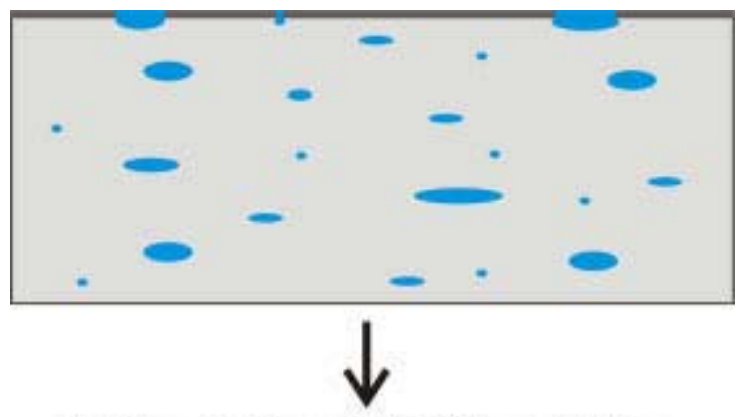

I. Dissolution of $\mathrm{Ni}_{5} \mathrm{Gd}$ particles

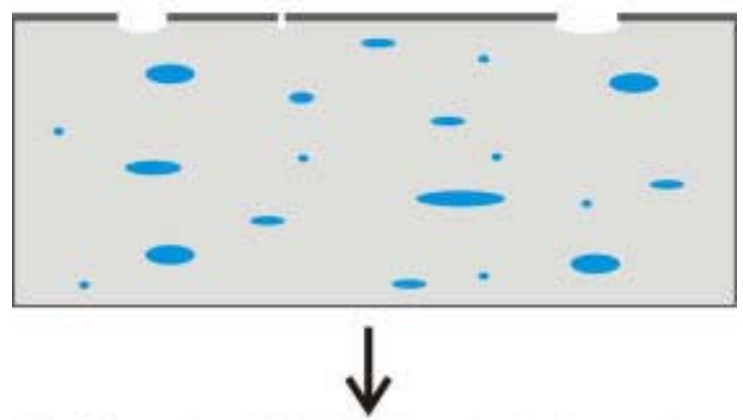

II. Growth of NiCrMo oxide in cavity

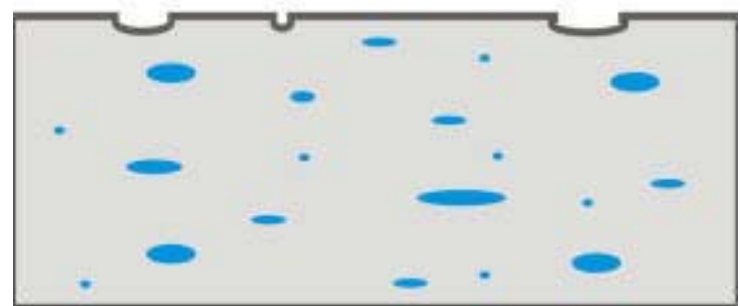

Figure 9. Diagram of Ni-Cr-Mo-Gd surface evolution during potentiostatic tests.

and as low as $0.233 \mathrm{Pm} / \mathrm{yr}$ (Test 062305) at 7 days. Testing at longer times than 7 days and at potentials that better define the likely corrosion potential would be desirable. Ideally, exposure test coupons would provide a better comparison case. Reference 19, Figure 7-1, also provides a time-based corrosion rate curve for Alloy 22 obtained through LPR and weight loss measurements in various simulated solutions. The average corrosion rate for cylindrical specimens $(1.27 \pm 0.60 \mathrm{Pm} / \mathrm{yr})$ after 7 days exposure for the Ni-Cr-Mo-Gd alloy fall above the trend line curve for the data and are similar to the LPR-derived corrosion rate for Alloy 22 after several minutes of immersion.

\subsection{Future Work}

While this alloy has shown promise in meeting the needs of a corrosion-resistant, neutron-absorbing alloy, more work is needed to investigate the unique corrosion characteristics of this alloy. This material has a unique two-phase structure where a highly reactive, gadolinide containing second phase is present in a corrosion resistant, austenitic matrix. Understanding this unique system is important to defending the corrosion properties of the material.

The corrosion properties of the secondary phase are very different than the base material and thus present a unique situation. Using traditional corrosion tests, the material does not have excellent performance as evidenced by the LPR data presented in Section 4.3. However, once the surface-exposed 
secondary phase is removed, the corrosion properties improve dramatically. While this improvement in performance has been documented in previous work, longer-term performance is yet to be examined. The secondary phase is still present in the material and will be exposed as the base material is corroded. Understanding how the subsurface secondary phase might be exposed and corroded is important. It is important to understand if there is a mechanism to penetrate deeper into the material. This is also a function of the microstructure of the alloy, and thus a careful study should consider a material that is in production form. The holes left by the removal of the secondary phase may act as crevices (limited mass transport). One method of approaching this problem would be to use more aggressive environments and long exposure times followed by sectioning analysis. Another would be to determine penetration rates through thin sections of material.

Other considerations for future work should consider pretreatments to remove the surface exposed secondary phase prior to performing the corrosion tests. The plate processing mill will use an acid pickling bath to remove the thick surface oxides that were formed during hot rolling and final annealing. This would also eliminate the issues with the exposed secondary phase observed in corrosion tests that have been discussed earlier.

Future work on this alloy should also include comparison testing of a standard commercial alloy with known corrosion performance. The target composition for the base metal for the Ni-Cr-Mo-Gd alloy is Alloy C-4. Thus comparison with this alloy would provide a greater level of understanding of how well the base material passivates after the secondary phase is removed. Knowing the corrosion rate of the base metal in the same environment without influence of the secondary phase will allow the residual effect of the secondary phase to be known.

Of some concern is the potential of fluoride ions to enhance crevice corrosion and needs to be ascertained. The concentration of fluoride ions are enhanced by leeching from vitrified waste within the waste package. Concentrations of fluoride in the High halide solution $(0.01 \mathrm{~m})$ are higher than the maximum chloride solution $(0.006 \mathrm{~m})$. The effect of this constituent needs to be further ascertained, particularly if elevated concentrations are expected in the waste package.

If the corrosion rates for the base composition alloy (ASTM 932B material) are not acceptable due to issues with crevice corrosion, investigation of higher chromium variants of the Ni-Cr-Mo-Gd alloys should be explored. There has been some promising work on an alloy having $21.01 \%$ chromium (see Reference 7). While crevice corrosion has not been studied for that alloy, it is anticipated that the performance should be greater than the material studied in this report. 


\section{REFERENCES}

1. ASTM B932-04, "Low-Carbon Nickel-Chromium-Molybdenum-Gadolinium Plate, Sheet, and Strip," 2004.

2. ASME Code Case N-728, "Use of B-932-04 Plate Material for Non-pressure Retaining Spent-fuel containment internals to $650^{\circ} \mathrm{F}\left(343^{\circ} \mathrm{C}\right)$, Section III, Division 3,” 2005.

3. DOE, Development of Gadolinium-Containing Stainless Steels, 2001, DOE/SNF/REP-066, Idaho National Engineering and Environmental Laboratory, January 2001.

4. R. E. Mizia, P. J. Pinhero, T. E. Lister, J. N. DuPont and C. V. Robino, "Corrosion Performance of a Gadolinium-Containing Stainless Steel,” Paper 138, Corrosion, NACE International, Houston, Texas, 2001.

5. J. N. Dupont, C. V. Robino, and R. E. Mizia, "Development of Gadolinium Containing Stainless Steels for Nuclear Criticality Control,” TMS 2000 Fall Meeting, TMS Society, St. Louis, Missouri, 2000.

6. R. E. Mizia, T. E. Lister, P. J. Pinhero, "Microstructure and Corrosion Performance of a Neutron Absorbing Ni-Cr-Mo-Gd Alloy,” Paper 679, Corrosion, NACE International, Houston, Texas, 2003.

7. T. E. Lister, R. E. Mizia, P. J. Pinhero, T. L. Trowbridge, and K. Delezene-Briggs, "Studies of the Corrosion Properties of Ni-Cr-Mo-Gd Neutron-Absorbing Alloys,” Corrosion, 61, 706, 2005.

8. R. E. Mizia, P. J. Pinhero, T. E. Lister, C. V. Robino, J. R. Michael, J. N. Dupont, and D. B. Williams, Interim Report on the Corrosion Performance of a Neutron Absorbing $\mathrm{Ni}-\mathrm{Cr}-\mathrm{Mo}-\mathrm{Gd}$ Alloy, DOE/SNF/REP-086, Rev. 0, National Spent Nuclear Fuel Program, March 2004.

9. R. A. Van Konynenburg, P. G. Curtis, and T. S. E. Summers, 1998, Scoping Corrosion Tests on Candidate Waste Package Basket Materials for the Yucca Mountain Project, UCRL-ID-130386, March 1998.

10. P. Pasupathi, White Paper, "Recommendations for Corrosion Rates of C4-Gd Neutron Absorber Alloy," April 12, 2004.

11. D. F. Fix, J. C. Estill, L. L. Wong, and R. B. Rebak, "General and Localized Corrosion of Austenitic and Borated Stainless Steels in Simulated Concentrated Ground Waters," UCRL-PROC-202920, Lawrence Livermore National Laboratory, March 15, 2004.

12. BSC, "Corrosion Testing of C4-Gd Alloy," BSC FY-05 Statement of Work, BSC Work Package Number AWPTA2.

13. PLN-1226, "Quality Program Plan: Corrosion Rate Testing of C4-Gd Neutron Absorbing Alloy," Idaho National Laboratory, Rev. 4 (latest revision), July 13, 2005.

14. PLN-1880, "Experimental Plan for Electrochemical Corrosion Testing of NiCr-Mo-Gd Alloys," Idaho National Laboratory PLN-1880, Rev. 1 (latest revision), July 13, 2005. 
15. PLN 1885, "Experimental Procedures for Electrochemical Testing of Ni-Cr-Mo-Gd Alloys," Idaho National Laboratory, Rev. 2 (latest revision), September 6, 2005.

16. ASTM G5-94 (reapproved 2004) "Standard Reference Test Method for Making Potentiostatic and Potentiodynamic Anodic Polarization Measurements," ASTM International, West Conshohocken, Pennsylvania.

17. CRC Handbook of Chemistry and Physics, $62^{\text {nd }}$ edition, CRC Press, Boca Raton, Florida, 1981, p. B-101.

18. A. Yilmaz, P. Pasupathi, and R. Rebak, "Stifling of Crevice Corrosion in Alloy 22 During Constant Potential Tests," ASME Pressure Vessel and Piping Division Conference, Denver, Colorado, 2005.

19. BSC, "General and Localized Corrosion of Waste Package Outer Barrier," ANL-EBS-MD-000003, Rev. 02, October 2004.

20. A. C. Lloyd, J. J. Noel, N. S. McIntyre, and D. W. Shoesmith, "The Open Circuit Ennoblement of C-22 and Other Ni-Cr-Mo Alloys," Journal of Metals, 57 (31), 2005.

21. R. E. Mizia, T. E. Lister, P. J. Pinhero, and T. L. Trowbridge, "Localized Corrosion of a Neutron Absorbing Ni-Cr-Mo-Gd Alloy," Paper 600, Corrosion, NACE International, Houston, Texas, 2005.

22. K. G. Mon, G. M. Gordon, and R. B. Rebak, "Stifling of Crevice Corrosion in Alloy 22," $12^{\text {th }}$ International Conference on Environmental Degradation in Nuclear Systems-Water Reactors, TMS, 2005.

23. R. D. McCright, Engineered Materials Characterization Report, Volume 3 Revision 1.1, "Corrosion Data and Modeling, Update for Via bility Assessment," URCL-ID-119564, Rev. 1.1, 1998, pp. 2.2-12 and 2.2-13. 


\section{Data Package and Explanation for Electrochemical Testing of Ni-Cr-Mo-Gd Alloys Conducted by INL in FY-05}

T. E. Lister

R. E. Mizia

S. M. Birk

October 2005

The INL is a U.S. Department of Energy National Laboratory operated by Battelle Energy Alliance 


\section{Explanation of the Data Package}

The data package is in the form of an Excel spreadsheet that contains both numerical data (i.e., corrosion rates) and observations made (i.e., damage to specimens) from the tests. The source of the information is contained in the scientific notebooks covering the work. The spreadsheet is split into four categories based on the type of specimen (crevice or rod) and the type of test (potentiodynamic [PD] and potentiostatic [PS]). The results are listed in the order they were performed. At the end of this document is an explanation of each data column for PD and PS data. All potentials are versus the saturated calomel electrode (SCE).

\section{Electrochemical testing sequences}

Potentiodynamic test sequence (in order)

Corrosion potential ( $\left.\mathrm{E}_{\mathrm{corr}}\right)$ measurement

50 minutes

Linear polarization resistance (LPR) measurement

$\pm 30 \mathrm{mV}$ of $\mathrm{E}_{\text {corr }}$

Potentiodynamic measurement (scan 1)

$\mathrm{E}_{\text {corr }}$ to 0.8 or $1.0 \mathrm{~V}$

Potentiodynamic measurement (scan 2)

$\mathrm{E}_{\text {corr }}$ to 0.8 or $1.0 \mathrm{~V}$

Potentiostatic test sequence (in order)

Corrosion potential $\left(\mathrm{E}_{\mathrm{corr}}\right)$ measurement

50 minutes

Potentiostatic measurement

$+0.2 \mathrm{~V}$ for 7 days

\section{Description of electrochemical tests and outputs}

Below are the four types of measurements and descriptions of the output parameters that are in the spreadsheet. For more information, consult Idaho National Laboratory (INL) document PLN-1885 that includes all the experimental procedures for the work.

\section{1) Corrosion potential $\left(\mathbf{E}_{\text {corr }}\right)$ measurement}

The corrosion potential was obtained immediately after immersion of the specimen. This was performed for 50 minutes for all tests. Four values were obtained:

\section{Output:}

Initial $\mathrm{E}_{\mathrm{corr}} \quad$ The first data value obtained

Final $\mathrm{E}_{\text {corr }} \quad$ The last data value obtained

Min $\mathrm{E}_{\mathrm{corr}} \quad$ The minimum value obtained

Max $\mathrm{E}_{\mathrm{corr}} \quad$ The maximum value obtained

\section{2) Linear polarization resistance (LPR) measurement}

The corrosion rate was calculated using the slope of the I-V curve (obtained using Echem Analyst Software V1.31). The calculation was performed using ASTM G59-97 Section 3 guidelines. The INL document PLN-1885, Procedure 2600 covers this calculation. LPR curves were obtained for PD tests only. A generic value of $0.0261 \mathrm{~V}$ was used for the Stern Geary Coefficient, a value based on generic Tafel slopes of $0.12 \mathrm{~V} / \mathrm{dec}$.

Output:

LPR CR The corrosion rate in $\mu \mathrm{m} / \mathrm{yr}$ 


\section{3) Potentiodynamic (PD) measurement}

Several parameters are obtained from the PD curves. The PD sweeps are split into two regions: 1) the passive region from the initiation value of the sweep to $0.6 \mathrm{~V}$ and 2) the transpassive region from $0.6 \mathrm{~V}$ to the anodic limit. The parameters obtained are explained below.

Output: (one set of values for each scan)

Pass Q Forward Charge passed from the initiation of the sweep to $0.6 \mathrm{~V}$.

Pass Q Reverse Charge passed from $0.6 \mathrm{~V}$ to the origin of the sweep.

Max I Passive Maximum current observed in forward or reverse sweep between initiation and $0.6 \mathrm{~V}$.

Trans Pass Q The charge (both forward and reverse) between $0.6 \mathrm{~V}$ and the anodic limit.

Max I Trans Pass The maximum current in forward or reverse sweep between $0.6 \mathrm{~V}$ and the anodic limit.

$\mathrm{E}_{\mathrm{rp}} \quad$ The repassivation potential. A definition of when the reverse sweep crosses the forward sweep was used to obtain this value in all tests. If no hysteresis is observed, them "none" was entered.

\section{4) Potentiostatic measurement}

The potentiostatic tests were performed for a period of 7 days at $0.2 \mathrm{~V}$ versus SCE. Several parame ters were obtained. Two corrosion rate values were calculated

A) Weight loss data: The calculation was performed using ASTM G31-72 (Reapproved 2004) Section 11. This is normally used for weight loss coupons but was adapted for use here. The INL document PLN-1885, Procedure 2300 describes this calculation. This was done for PS test data only.

B) Icorr: The calculation was performed using ASTM G102 Section 4.7 guidelines. The INL document PLN-1885, Procedure 2300 covers this calculation. This was performed for PS test data only.

\section{Output:}

Total Charge

Peak Current

$\mathrm{I}_{\text {corr }}$

CR

CR WL
The entire charge passed during the experiment

The maximum current obtained during the test

The final current point of the PS test

Corrosion rate obtained from $\mathrm{I}_{\text {corr }}$

Corrosion rate obtained from weight loss.

\section{Post-test solution analysis}

The analysis of the post test solution was performed for the main elemental constituents (Ni, Cr, Mo, and Gd) by ICP-MS using a qualified laboratory. The results of the data are reported on the data sheets for each test in $\mu \mathrm{g} / \mathrm{L}$ (the reported value). A calculation was performed to determine the total dissolved metal ion mass in solution in $\mathrm{mg} / \mathrm{L}$, which can be compared to the weight lost by the specimen. Note that precipitation was observed in some tests. 


\section{Specimen weight loss}

The weight lost during the test was determined by measuring before (after degreasing) and after the test (after descaling). This weight is reported for all tests. The weight loss is used to determine a corrosion rate for PS tests only.

\section{Other reported data}

These are contained on separate sheets within the data spreadsheet.

\section{1) Equivalent weight}

The calculation of $\mathrm{EW}$ (equivalent weight) was required for corrosion rate determinations. This was made one time for the material using ASTM G102 Sections 4.2 through 4.6. The corrosion products were the values expected $\left(\mathrm{Ni}^{+2}, \mathrm{Cr}^{+3}, \mathrm{Mo}^{+3}, \mathrm{Gd}^{+3}\right)$. The equivalent weight $(27.1 \mathrm{~g})$ was used in all these calculations.

\section{2) Initial solution composition}

The initial anion compositions $\left(\mathrm{Cl}, \mathrm{NO}_{3}{ }^{-}\right.$and $\left.\mathrm{F}^{-}\right)$and $\mathrm{pH}$ are reported for each solution used in testing. The conversion to molal is also provided. Only one batch of solution was made for each solution type. 


\section{Potentiodynamic data}

\begin{tabular}{|c|c|c|}
\hline Col & $\underline{\text { Label }}$ & Description \\
\hline A) & $\overline{\text { Test ID }}$ & $\overline{\text { Unique test ID number }}$ \\
\hline B) & Sample ID & Unique sample ID number \\
\hline C) & Heat & Heat number for the sample \\
\hline D) & Matrix & Condition from PLN-1880 Appendix A \\
\hline E) & Solution & Solution name from PLN-1880 Appendix A \\
\hline F) & Temp & Test temperature \\
\hline G) & Wt loss (mg) & Weight lost during test \\
\hline $\mathrm{H})$ & Ecorr test duration & Length of $E_{\text {corr }}$ test (in minutes) \\
\hline I) & Initial Ecorr (V) & Initial measured $\mathrm{E}_{\mathrm{corr}}$ \\
\hline J) & Final Ecorr (V) & Final measured $\mathrm{E}_{\text {corr }}$ \\
\hline K) & Min Ecorr (V) & Lowest measured $\mathrm{E}_{\text {corr }}$ \\
\hline L) & Max Ecorr (V) & Highest measured $\mathrm{E}_{\mathrm{corr}}$ \\
\hline M) & LPR CR $(\mu \mathrm{m} / \mathrm{yr})$ & Corrosion rate calculated from LPR curve \\
\hline $\mathrm{N})$ & PD test anodic limit & Reversal potential for PD test \\
\hline O) & Pass Q Forward $1\left(\mathrm{C} / \mathrm{cm}^{2}\right)$ & Charge passed from $\mathrm{E}_{\text {corr }}$ to $0.6 \mathrm{~V}$ in forward sweep \\
\hline P) & Pass Q Reverse $1\left(\mathrm{C} / \mathrm{cm}^{2}\right)$ & Charge passed from $\mathrm{E}_{\text {corr }}$ to $0.6 \mathrm{~V}$ in reverse sweep \\
\hline Q) & Max I Passive $1\left(\mathrm{~A} / \mathrm{cm}^{2}\right)$ & Max current from $\mathrm{E}_{\text {corr }}$ to $0.6 \mathrm{~V}$ (forward or reverse) \\
\hline $\mathrm{R})$ & Trans Pass Q $1\left(\mathrm{C} / \mathrm{cm}^{2}\right)$ & Charge passed from $\mathrm{E}_{\text {corr }}$ to $0.6 \mathrm{~V}$ in reverse sweep \\
\hline S) & Max I Trans Pass $1\left(\mathrm{~A} / \mathrm{cm}^{2}\right)$ & Max current from $\mathrm{E}_{\text {corr }}$ to $0.6 \mathrm{~V}$ (forward or reverse) \\
\hline $\mathrm{T})$ & $\operatorname{Erp} 1(\mathrm{~V})$ & Repassivation potential measured using crossover criteria \\
\hline U) & Pass Q Forward $2\left(\mathrm{C} / \mathrm{cm}^{2}\right)$ & Charge passed from $E_{\text {corr }}$ to $0.6 \mathrm{~V}$ in forward sweep \\
\hline V) & Pass Q Reverse $2\left(\mathrm{C} / \mathrm{cm}^{2}\right)$ & Charge passed from $\mathrm{E}_{\text {corr }}$ to $0.6 \mathrm{~V}$ in reverse sweep \\
\hline W) & Max I Passive $2\left(\mathrm{~A} / \mathrm{cm}^{2}\right)$ & Max current from $\mathrm{E}_{\text {corr }}$ to $0.6 \mathrm{~V}$ (forward or reverse) \\
\hline $\mathrm{X})$ & Trans Pass Q $2\left(\mathrm{C} / \mathrm{cm}^{2}\right)$ & Charge passed from $\mathrm{E}_{\text {corr }}$ to $0.6 \mathrm{~V}$ in reverse sweep \\
\hline Y) & Max I Trans Pass $2\left(\mathrm{~A} / \mathrm{cm}^{2}\right)$ & Max current from $\mathrm{E}_{\text {corr }}$ to $0.6 \mathrm{~V}$ (forward or reverse) \\
\hline Z) & Erp $2(\mathrm{~V})$ & Repassivation potential measured us ing crossover criteria \\
\hline AA) & EC Comments & Observations from EC test data \\
\hline $\mathrm{AB})$ & Solution Observations & Observations of solution after test \\
\hline $\mathrm{AC})$ & Specimen Observations & Observations of specimen after test \\
\hline $\mathrm{AD})$ & LOM Observations & Observations from LOM analysis \\
\hline $\mathrm{AE})$ & SEM Observations & Observations from SEM analysis \\
\hline $\mathrm{AF})$ & Solution Analysis Ni ug/L & Concentration of $\mathrm{Ni}$ from solution analysis \\
\hline $\mathrm{AG})$ & Solution Analysis Cr ug/L & Concentration of $\mathrm{Ni}$ from solution analysis \\
\hline $\mathrm{AH})$ & Solution Analysis Mo ug/L & Concentration of $\mathrm{Ni}$ from solution analysis \\
\hline $\mathrm{AI})$ & Solution Analysis Gd ug/L & Concentration of $\mathrm{Ni}$ from solution analysis \\
\hline \multirow[t]{2}{*}{$\mathrm{AJ})$} & Total Solution Analysis & \\
\hline & wt mg/L & Total concentration of metal ions \\
\hline $\mathrm{AK})$ & $\mathrm{pH}$ & Measured solution $\mathrm{pH}$ of solution after test \\
\hline AL) & Test ID & Unique test ID number (repeated) \\
\hline AM) & Verified & Checked against reviewed notebook data \\
\hline
\end{tabular}




\section{Potentiostatic data}

\begin{tabular}{|c|c|c|}
\hline Col & $\underline{\text { Label }}$ & Description \\
\hline A) & Test ID & Unique test ID number \\
\hline B) & Sample ID & Unique sample ID number \\
\hline C) & Heat & Heat number for the sample \\
\hline D) & Matrix & Condition from PLN-1880 Appendix A \\
\hline E) & Solution & Solution name from PLN-1880 Appendix A \\
\hline F) & Temp & Test temperature \\
\hline G) & Wt Loss (mg) & Weight lost during test \\
\hline H) & CR WL $(\mu \mathrm{m} / \mathrm{yr})$ & Corrosion rate calculated from weight loss \\
\hline I) & Ecorr test duration & Length of $\mathrm{E}_{\text {corr }}$ test (in minutes) \\
\hline J) & Initial Ecorr (V) & Initial measured $\mathrm{E}_{\text {corr }}$ \\
\hline K) & Final Ecorr (V) & Final measured $\mathrm{E}_{\mathrm{corr}}$ \\
\hline L) & Min Ecorr (V) & Lowest measured $\mathrm{E}_{\text {corr }}$ \\
\hline M) & Max Ecorr (V) & Highest measured $\mathrm{E}_{\mathrm{corr}}$ \\
\hline N) & PS test duration (hours) & Length of PS test in hours \\
\hline O) & PS test potential (V) & Potential the specimen was held during test \\
\hline P) & Total Charge $\left(\mathrm{C} / \mathrm{cm}^{2}\right)$ & The total charge passed during PS test \\
\hline Q) & Peak Current $\left(\mathrm{A} / \mathrm{cm}^{2}\right)$ & The maximum current observed during test \\
\hline R) & Icorr $\left(\mathrm{A} / \mathrm{cm}^{2}\right)$ & The final current measured during PS test \\
\hline S) & $\mathrm{CR}(\mu \mathrm{m} / \mathrm{yr})$ & The corrosion rate calculated from $\mathrm{I}_{\text {corr }}$ \\
\hline T) & EC Comments & Observations from EC test data \\
\hline U) & Solution Observations & Observations of solution after test \\
\hline V) & Specimen Observations & Observations of specimen after test \\
\hline W) & LOM Observations & Observations from LOM analysis \\
\hline $\mathrm{X})$ & SEM Observations & Observations from SEM analysis \\
\hline Y) & Solution Analysis Ni ug/L & Concentration of $\mathrm{Ni}$ from solution analysis \\
\hline Z) & Solution Analysis Cr ug/L & Concentration of $\mathrm{Ni}$ from solution analysis \\
\hline AA) & Solution Analysis Mo ug/L & Concentration of Ni from solution analysis \\
\hline $\mathrm{AB})$ & Solution Analysis Gd ug/L & Concentration of $\mathrm{Ni}$ from solution analysis \\
\hline \multirow[t]{2}{*}{$\mathrm{AC})$} & Total Solution Analysis & \\
\hline & wt mg/L & Total concentration of metal ions \\
\hline $\mathrm{AD})$ & $\mathrm{pH}$ & Measured solution $\mathrm{pH}$ of solution after test \\
\hline $\mathrm{AE})$ & Test ID & Unique test ID number \\
\hline AF) & Verified & Checked against reviewed notebook data \\
\hline
\end{tabular}




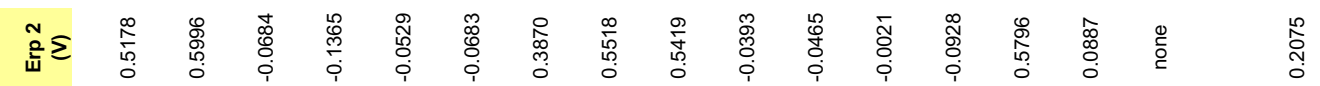

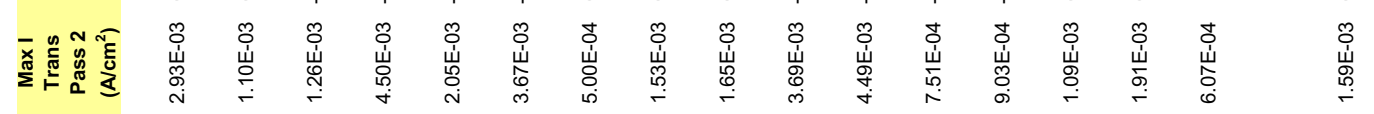

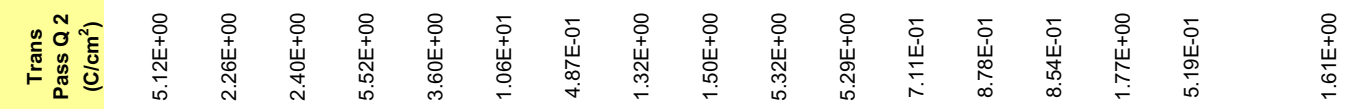

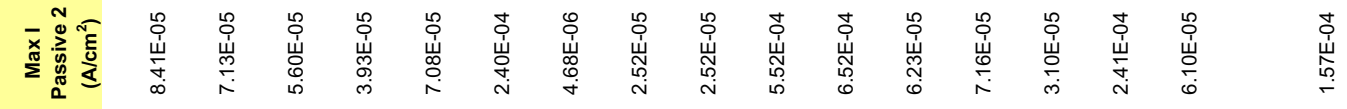

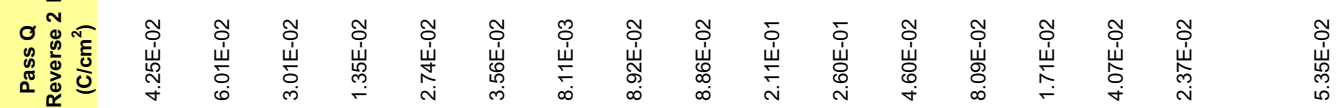

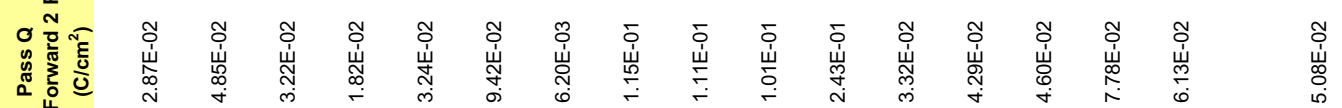

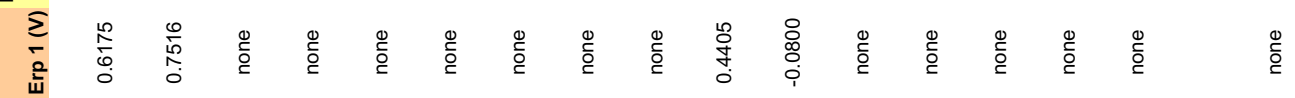

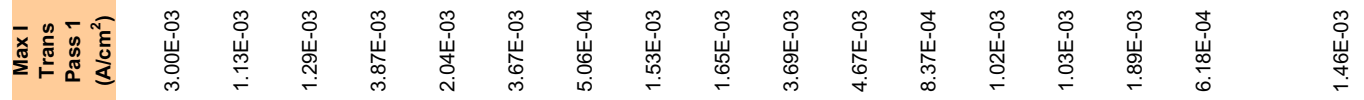

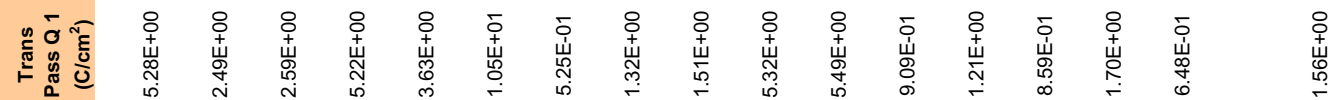

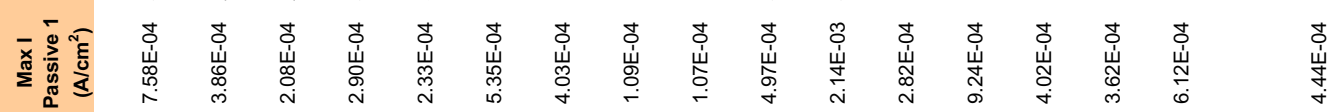

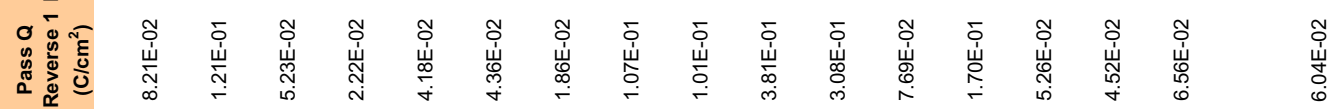

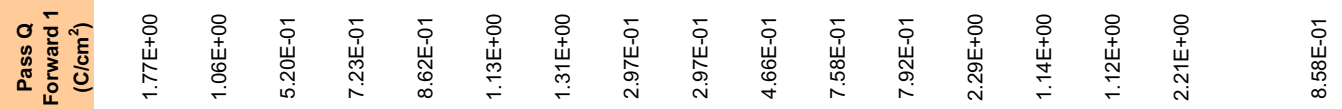

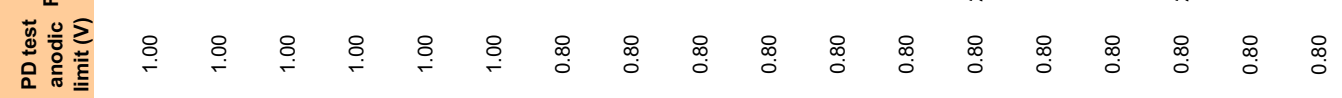

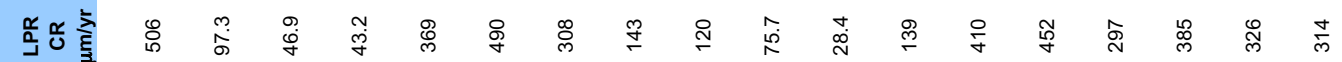

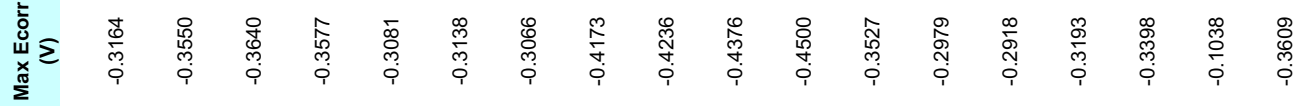

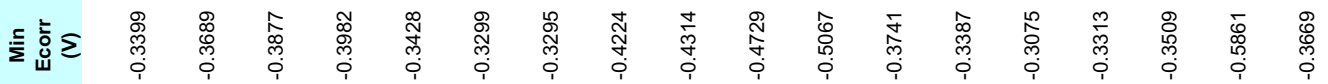

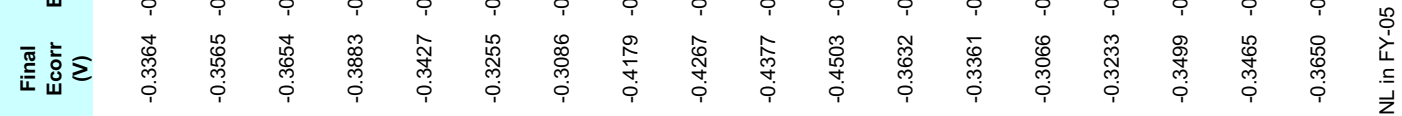

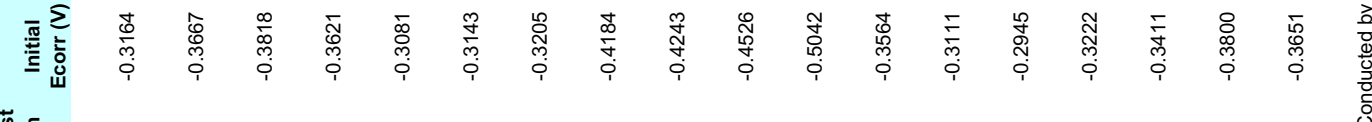

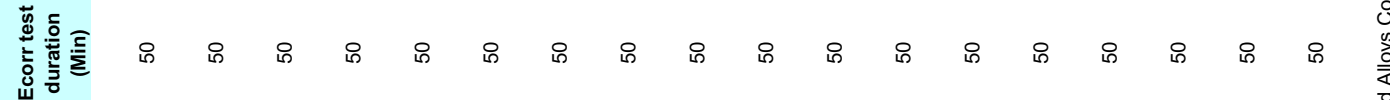

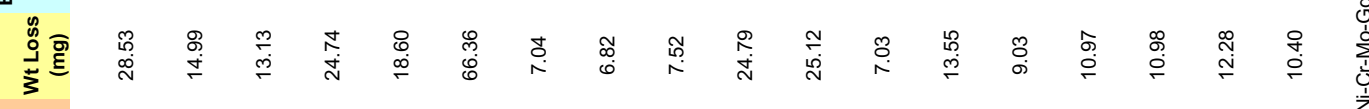

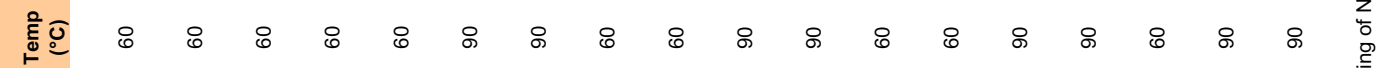

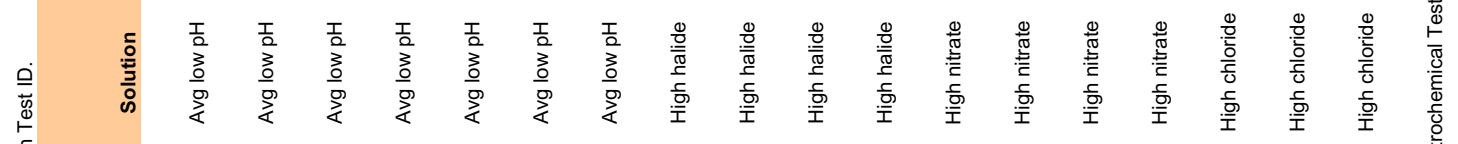

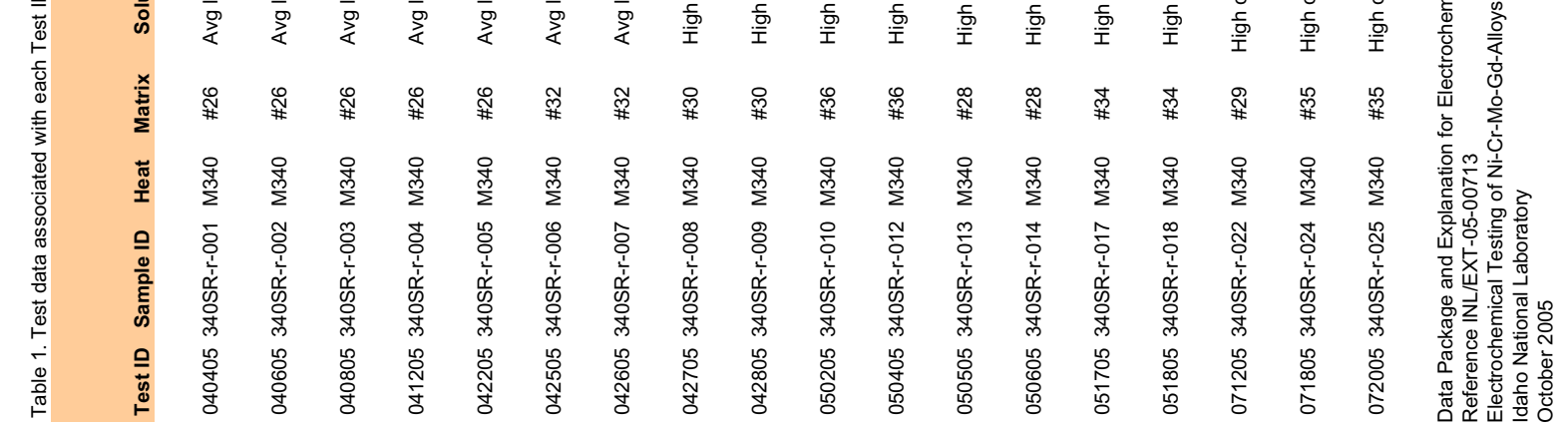




\section{I}

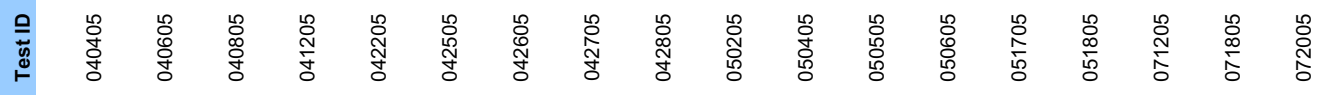

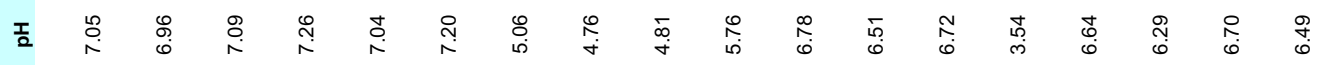

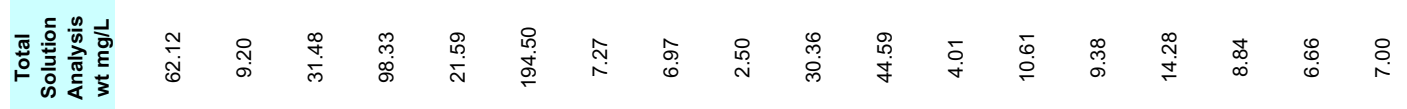

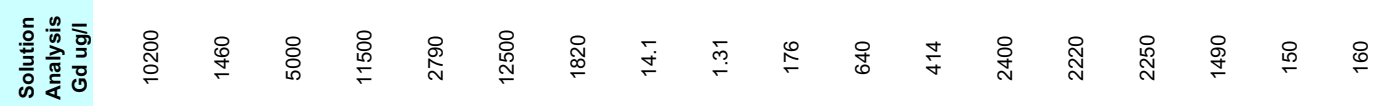

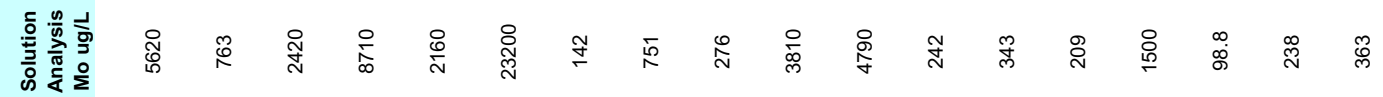

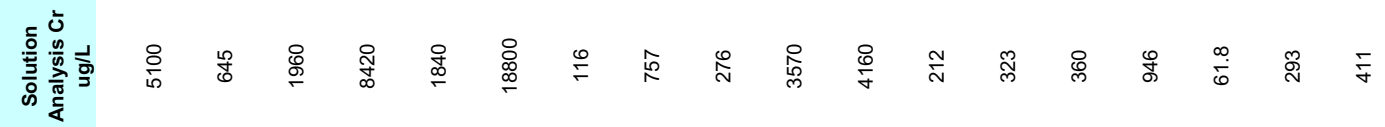

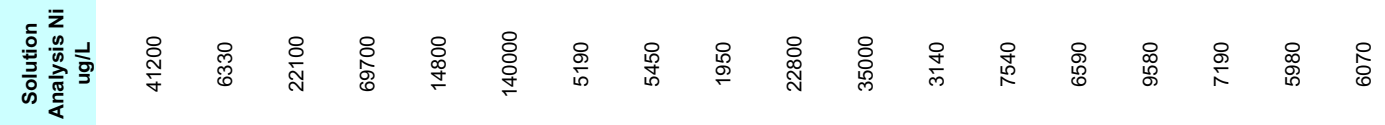

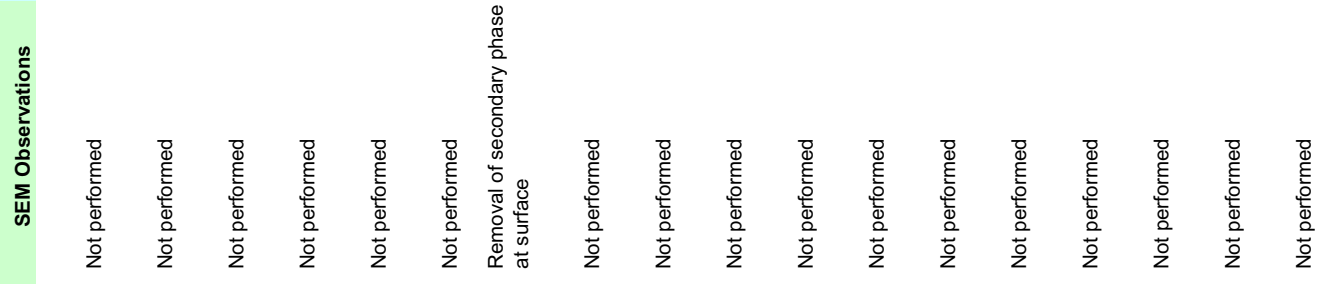

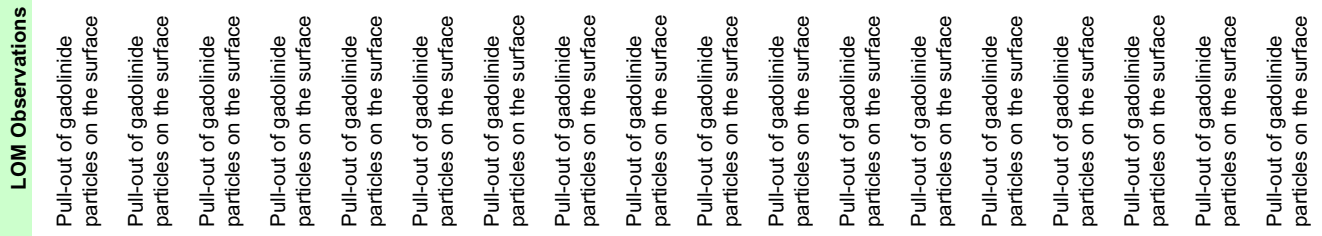

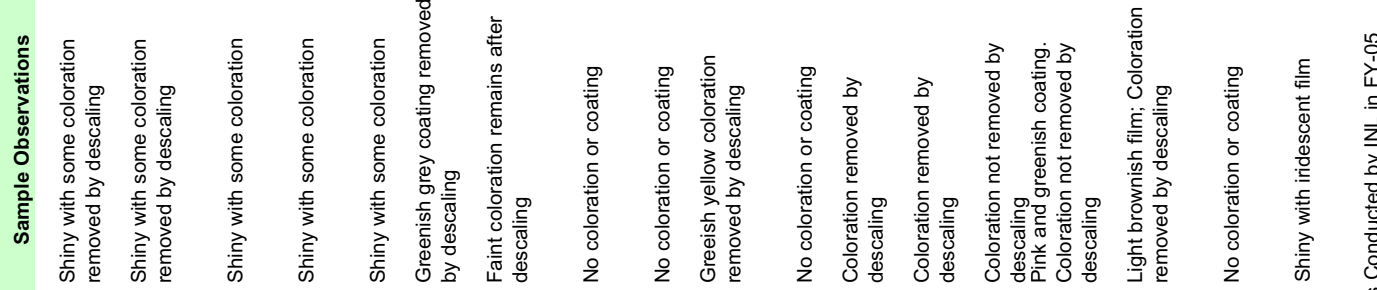

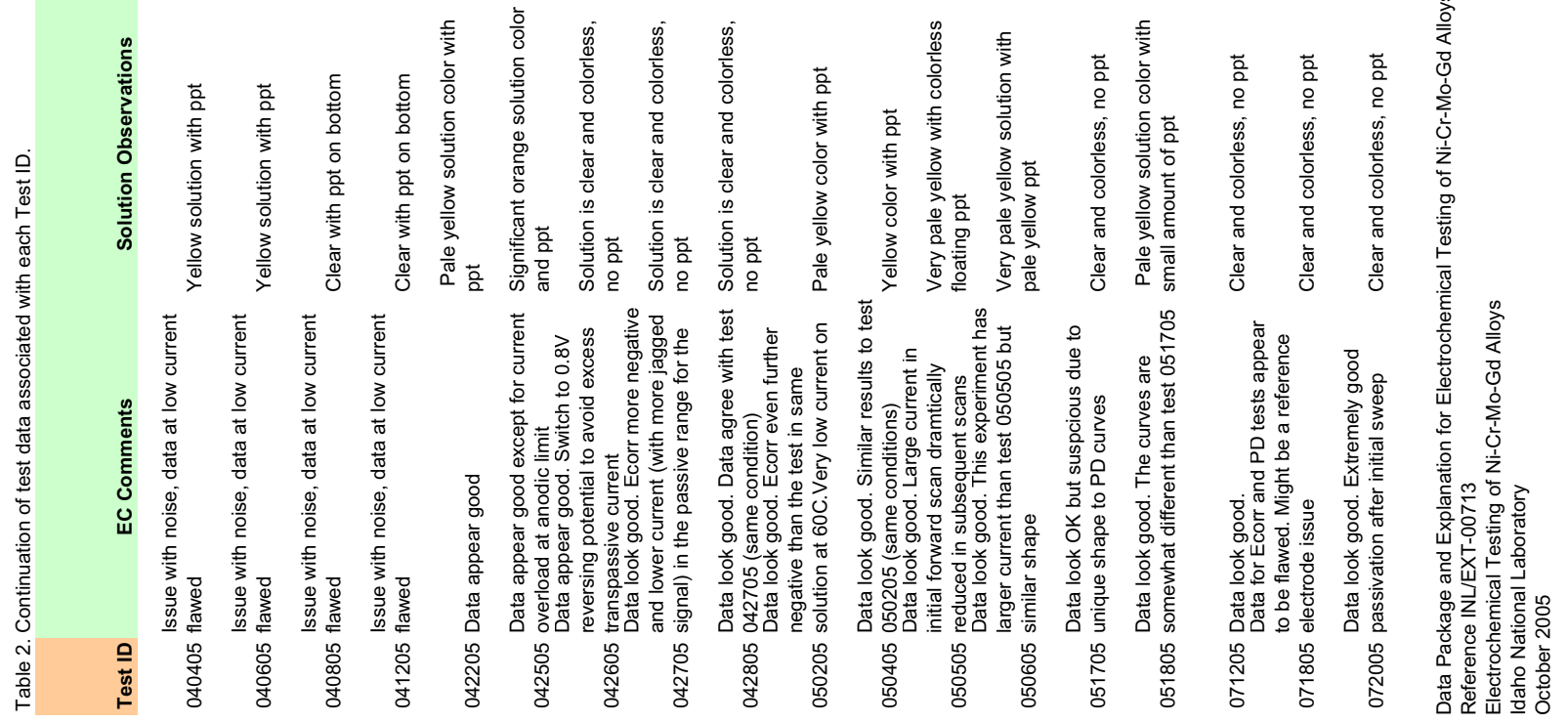

